\title{
Phytosociological analysis of noble hardwood forests (Ostryo-Tilienion platyphylli) in the Karst and its neighbouring regions (SW Slovenia)
}

\author{
Igor Dakskobler ${ }^{1}$ \& Livio Poldini ${ }^{2}$
}

Key words: phytosociology, synsystematics, Tilio-Acerion, OstryoTilienion platyphylli, Natura 2000, Karst, Slovenia.

Ključne besede: fitocenologija, sinsistematika, Tilio-Acerion, OstryoTilienion platyphylli, Natura 2000, Kras, Slovenija.

\begin{abstract}
In SW Slovenia, at Gora under Železna Vrata and Petnik gorge near Branik both on the northern edge of the Karst, in the collapse doline Orleška Draga at Sežana and under Brkinski Rob at the contact of the Karst and Brkini Hills, we conducted a phytosociological analysis of the stands whose tree layer is dominated by Tilia platyphyllos, T. cordata, Acer pseudoplatanus, Ulmus glabra, in places also Carpinus betulus and Ostrya carpinifolia, and classified them into three associations, Corydalido ochroleucae-Aceretum pseudoplatani, Paeonio officinalis-Tilietum platyphylli and Fraxino orni-Aceretum pseudoplatani. The latter was described as a new. Also new is a secondary large-leaved lime association Lamio orvalae-Tilietum platyphylli on the sites of montane beech forests (Lamio orvalae-Fagetum) in the Cepovan valley, at the contact of the Dinaric and subMediterranean phytogeographical regions of Slovenia.

\section{Izvleček}

V jugozahodni Sloveniji: Gora pod Železnimi vrati in grapa Petnik pri Braniku, oboje na severnem robu Krasa, v udornici Orleška draga pri Sežani in pod Brkinskim robom na stiku Krasa in Brkinov smo fitocenološko analizirali sestoje, v katerih v drevesni plasti prevladujejo vrste Tilia platyphyllos, T. cordata, Acer pseudoplatanus, Ulmus glabra, ponekod tudi Carpinus betulus in Ostrya carpinifolia in jih uvrstili v tri asociacije Corydalido ochroleucae-Aceretum pseudoplatani, Paeonio officinalis-Tilietum platyphylli in Fraxino orni-Aceretum pseudoplatani. Slednjo smo opisali kot novo. Prav tako je nova drugotna lipova asociacija Lamio orvalae-Tilietum platyphylli na rastiščih gorskih bukovih gozdov (Lamio orvalae-Fagetum) v Čepovanski dolini, na stiku dinarskega in submediteranskega fitogeografskega območja Slovenije.
\end{abstract}

Received: 26.9 .2020

Revision received: 23. 3. 2021

Accepted: 27. 4. 2021

\footnotetext{
1 Scientific Research Centre of the Slovenian Academy of Sciences and Arts, Institute of Biology, Regional Unit Tolmin, Brunov drevored 13, SI-5220 Tolmin, Slovenia. E-mail: igor.dakskobler@zrc-sazu.si

2 Department of Life Sciences, University of Trieste, via L. Giorgieri, 5, I-34127 Trieste, Italy. E-mail: poldini@units.it
} 


\section{Introduction}

Noble hardwood forests (sycamore and Norway maple, wych elm, large-leaved and small-leaved lime) with admixture of thermophilic deciduous trees (hop hornbeam, flowering ash) that are classified into the alliance Tilio-Acerion and suballiance Ostryo-Tilienion platyphylli (Košir et al. 2008) have until now only been known on very small areas in the Karst (Dakskobler 2006, Dakskobler \& Reščič 2015). They cover slightly bigger areas in contact regions in Čičarija and Vremščica (and were researched by Accetto 1991, Košir 2004, Košir \& Surina 2005). In our research in recent years we identified such stands also on the northern edge of the Karst (Gora under Železna Vrata, Petnik gorge at Branik), in Orleška Draga and under Brkinski Rob (under the ridge between Knežak and Ratečevo Brdo). Pioneer large-leaved lime stands were recorded also in the Čepovan Valley, slightly more to the north - Figure 1. Some of these areas were mapped in the framework of a target research project (Šilc et al. 2017), but until now they have not been subject to a detailed phytosociological analysis or classified into a syntaxonomic system. This was the aim of the present study.

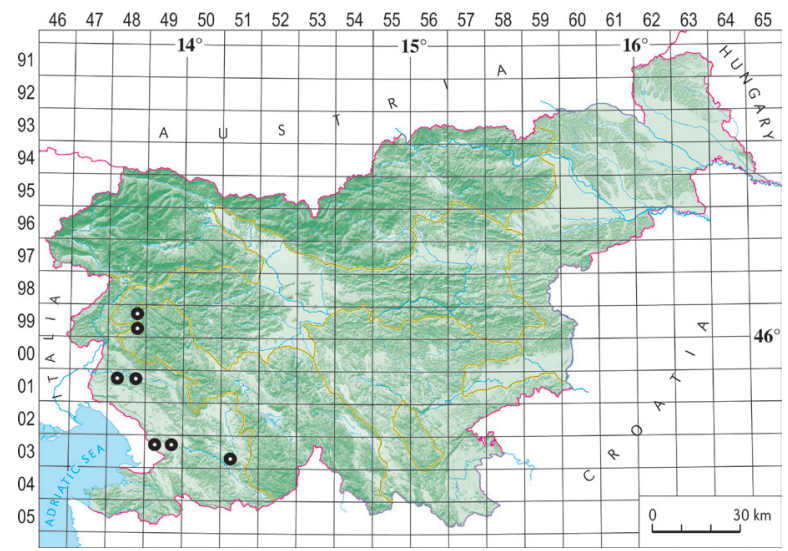

Figure 1: Localities of the researched Tilia platyphyllos and Acer pseudoplatanus stands in western and southwestern Slovenia. Slika 1: Nahajališča raziskanih lipovih in javorovih sestojev v zahodni in jugozahodni Sloveniji.

\section{Methods}

Forest stands of noble hardwoods in the Karst and neighbouring regions were researched using the standard Central-European method (Braun-Blanquet 1964). The relevés were made in the spring of 2009, 2014, 2016, 2018 and 2019 and are stored in the FloVegSi database (Seliškar et al. 2003). We started with a table into which we arranged 46 relevés from five research areas, namely Gora under Železna Vrata, Petnik gorge, Orleška Draga,
Risnik and Brkinski Rob, and included also several relevés that were not dominated by noble hardwoods, but were made at the contact with their stands. These relevés were mutually compared using unweighted average linkage (UPGMA) hierarchical clustering method and Wishart's similarity ratio. Combined cover-abundance values were converted into the ordinal scale 1 to 9 (van der Maarel 1979). We used the programme package SYN-TAX 2000 (Podani 2001). Based on the resulting clusters of relevés we made four analytical tables followed by a synthetic table, in which we took into account only the relevés that grouped into clusters and were identified as belonging to the alliance Tilio-Acerion and suballiance Ostryo-Tilienion. The stands in the Čepovan valley were analysed separately. In describing the two new associations we considered not only the entire species composition, but also the structure of the upper stand layer, i.e. the dominant tree species. The nomenclatural source for the names of vascular plants is Martinčič et al. (2007). Some new findings, for example taxon Helleborus dumetroum Waldst. \& Kit. ex Willd. subsp. illyricus Starmühler (Rottensteiner 2016) which is similar to taxon H. multifidus subsp. istriacus and Crocus heuffellianus Herb. (Peruzzi 2016) instead of taxon Crocus vernus subsp. vernus are in our tables not considered, but mentioned only as a note in the end of them. Martinčic $(2003,2011)$ is the nomenclatural source for the names of mosses, and Suppan et al. (2000) for the names of lichens. The source for the nomenclature of soil types was Urbančič et al. (2005). The nomenclatural source for the names of syntaxa are Šilc \& Čarni (2012), except for the name of the class Querco-Fagetea Braun-Blanquet et Vlieger in Vlieger 1937. The sources of climate data for the study area are Ogrin (1996), B. Zupančič (1998) and Cegnar (1998), whereas Buser (2009) served as the source for geological data.

\section{Ecological description of the study area}

All five study areas in the Karst and its fringes have hinterland sub-Mediterranean climate and the stands in the Čepovan valley are in the region of the temperate continental climate of western and southern Slovenia. The difference is in the annual precipitation volume, which is slightly higher (1600 to $1800 \mathrm{~mm}$ ) in the northern edge of the Karst above the Vipava Valley, whereas other study areas receive between 1400 and $1500 \mathrm{~mm}$. The differences in mean annual air temperature are less obvious, because Risnik and Orleška Draga are collapse dolines with persistent cold air pools, where mean air temperature is 
different from the average in the surroundings of Sežana (which is $10-12^{\circ} \mathrm{C}$ ). The same average $\left(10-12^{\circ} \mathrm{C}\right.$ ) is on the Karst slopes above the Vipava Valley, but the local climate there is colder because of northern aspects and gorges (Petnik). Ratečevo Brdo or Brkinski Rob, however, is in the region with mean annual temperature between $8{ }^{\circ} \mathrm{C}$ and $10^{\circ} \mathrm{C}$. The Čepovan valley has a lower mean annual temperature $\left(6-8{ }^{\circ} \mathrm{C}\right)$ than other areas and higher mean annual precipitation volume (about $2000 \mathrm{~mm}$ ). The parent material in Risnik and Orleška Draga is limestone and its talus slopes, under Gora at Železna Vrata it is limestone and marlstone with their scree, in Petnik gorge rockfall, talus cones, limestone and marlstone rubble, and under Brkinski Rob limestone and its rubble, with insignificant admixture of marlstone. Limestone and dolomite are the predominant parent material in the Čepovan valley.

\section{Results and discussion}

\section{Hierarchical classification of relevés and their comparison}

In hierarchical clustering, 46 relevés from five study areas were grouped into two large clusters, each with two subclusters. A few of the relevés stood out from the rest (Figure 2).

Based on the results of this classification the relevés were arranged into a single table, which was further divided into four tables, one for each of the four groups.

\section{Corydalido ochroleucae-Aceretum pseudoplatani}

Table 1 comprises 12 relevés of the first cluster (left side of the dendrogram in Figure 2), that were made in Orleška Draga and Risnik. Based on the soil conditions and floristic composition relevés 11 and 12 from this table cannot be classified into the alliance Tilio-Acerion. Relevé 11 (Risnik) correspond to the subassociations Asaro-Carpinetum betuli dentarietosum enneaphylli (Poldini 1985), but relevé 12 (Orleška Draga) is, because of high abundance of Lamium orvala, classified as a new subassociation Asaro-Carpinetum betuli lamietosum orvalae subass. nov. hoc loco. Although the relevés from Orleška Draga grouped separately from the Risnik relevés and did not comprise Pseudofumaria alba, the results of hierarchical classification indicate that they can be classified into the same subassociation, i.e. Corydalido ochroleucae-Aceretum pseudoplatani Acetto 1991 veratretosum nigri Dakskobler et Reščič 2015, but we revised the differential species of the subassociation. Fraxinus excelsior is not a differential species of the subassociation, but a differential species of the new variant (var. Fraxinus excelsior), into which we classify the Risnik relevés. The differential species of this variant include Aconitum lycoctonum and Hacquetia epipactis, whereas Campanula rapunculoides can be considered a differential species of the subassociation. The stands from Orleška Draga are classified into the new variant with Isopyrum thalictroides. Its differential species are also Viola reichenbachiana, Acer campestre, Mercurialis ovata and Melica uniflora.

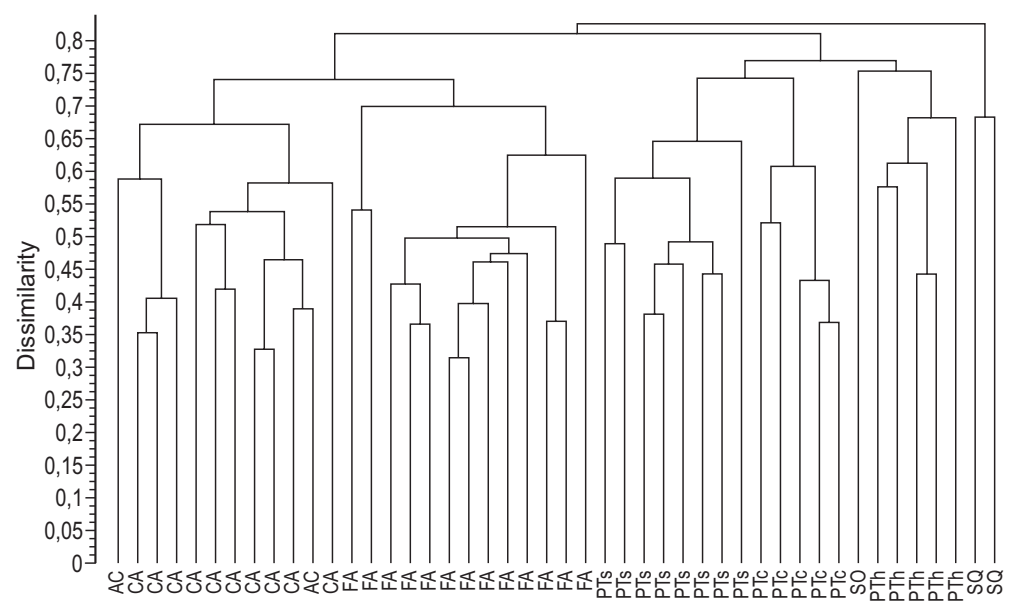

Figure 2: Dendrogram of relevés of communities with dominant Tilia platyphyllos and Acer pseudoplatanus in the Karst and neighbouring regions (UPGMA, 1- similarity ratio).

Slika 2: Dendrogram popisov združb plemenitih listavcev na Krasu in sosednjih območjih (UPGMA, komplement Wishartovega koeficienta podobnosti).

\section{Legend / Legenda}

AC Asaro-Carpinetum betuli

CA Corydalido ochroleucae-Aceretum pseudoplatani

FA Fraxino orni-Aceretum pseudoplatani

PTs Paeonio officinalis-Tilietum platyphylli saxifragetosum rotundifoliae
PTc Paeonio officinalis-Tilietum platyphylli castaneetosum sativae PTh Paeonio officinalis-Tilietum platyphylli hierochloetosum australis

SO Seslerio autumnalis-Ostryetum

SQ Seslerio autumnalis-Quercetum cerridis/Asaro-Carpinetum betuli 
These stands are distinguished from the Risnik relevés also by a smaller proportion of sycamore and large-leaved lime, a higher proportion of small-leaved lime and higher mean cover of common hornbeam in the tree layer. Prof. Andrej Rozman found a copy of the table of the association Carpinetum orientalis in Prof. Dušan Robičss legacy. The table is from the original manuscript of Prof. Gabrijel Tomažič, who lent the table to D. Robič in April 1968 as a teaching aid for students of phytosociology. This manuscript table includes six relevés from Orleška Draga, where oriental hornbeam (Carpinus orientalis) is recorded with a cover value 1 to 3 in the tree layer. Sessile oak (Quercus petraea) has a similar value and common hornbeam (Carpinus betulus) occurs only sporadically, with a cover value +. Fraxinus ornus and Acer campestre are more frequent, but sycamore (Acer pseudoplatanus) is very rare. Large and small-leaved lime and wych elm are completely absent in these relevés. At present, the forest in Orleška Draga is completely different. Oriental hornbeam was recorded only on two sites. It was not reported for this area in detailed mapping of the flora of the Gorizia-Trieste Karst region (Poldini 2009). Similarly, the entire species composition of our relevés is also completely different from Prof. Tomažičs's relevés. The reason could be that our relevés were not made on the same localities - with the state border running across this collapse doline the area probably was not easily accessible after World War II and Tomažič may have made his relevés somewhere in the vicinity.

\section{Fraxino orni-Aceretum pseudoplatani}

Relevés in the second cluster (13 relevés on the left side of the dendrogram in Figure 1) were made in Petnik gorge near Branik. Based on their entire species composition these relevés cannot be classified into the same association as the stands from Risnik and Orleška Draga, i.e. into the association Corydalido ochroleucae-Aceretum pseudoplatani, nor into the large-leaved lime community Paeonio-Tilietum platyphylli. They are the most similar to the stands of the association Veratro nigri-Fraxinetum excelsioris, whose fragments have been reported also at Škocjan Caves in the Karst (Dakskobler 2006), and with higher frequency in the Central Soča Valley (Dakskobler 2007). Two stands in the Škocjan Caves are dominated by largeleaved lime (Tilia platyphyllos) in the tree layer, where ash (Fraxinus excelsior) also occurs in one relevé, whereas sycamore (Acer pseudoplatanus) occurs only in the shrub layer. In terms of their entire species composition these two stands could also be classified into the subassociation Corydalido ochroleucae-Aceretum pseusoplatani veratretosum nigri. However, we did not record Fraxinus excelsior in our stands, and Veratrum nigrum was recorded only in one relevé. Based on the species dominating the herb layer (Lamium orvala) and the tree layer (Acer pseudoplatanus) they should be classified into the association Lamio orvalae-Aceretum pseudoplatani, but this name already designates an altimontane maple community from the Slovenian and the Dinaric Alps (Košir 2005) which does not belong to the suballiance Ostryo-Tilienion, but to the Lunario-Acerenion pseudoplatani. Similarly, Rusco aculeatiAceretum pseudoplatani would be an appropriate name for these stands, if it had not already been used for ecologically and phytogeographically entirely different stands in the Channel Islands along the coast of Normandy, France (Gehú 1997). They are therefore classified into the new association Fraxino orni-Aceretum pseudoplatani ass. nov. hoc loco. Its diagnostic species are Acer pseudoplatanus, Ulmus glabra, Fraxinus ornus, Lamium orvala, Ruscus aculeatus and Asparagus acutifolius. They characterise mixed colline (elevations between $150 \mathrm{~m}$ and $235 \mathrm{~m}$ ) sycamore (Acer pseudoplatanus) and wych elm (Ulmus glabra) forest with occasional admixture of field maple (Acer campestre), European hop-hornbeam (Ostrya carpinifolia), manna ash (Fraxinus ornus), common hornbeam (Carpinus betulus), large-leaved lime (Tilia platyphyllos), black locust (Robinia pseudoacacia), and very rarely also chestnut (Castanea sativa), small-leaved lime (Tilia cordata) and beech (Fagus sylvatica), frequently with the climber Hedera helix in the deep gorge, mainly on rockfall and slope scree with colluvial soils and rendzina. The lower shrub layer and the herb layer are dominated by Ruscus aculeatus, Lamium orvala, Polystichum setiferum, Phyllitis scolopendrium, Isopyrum thalictroides, Lunaria rediviva, Galanthus nivalis, Cardamine enneaphyllos, C. bulbifara, Mercurialis perennis and Corydalis cava, and the moss layer by Thamnobryum alopecurum and Isothecium alopecuroides. This is a special forest community on mixed, limestone-marl (flysch) bedrock in a colder local variant of the warm sub-Mediterranean climate. The nomenclatural type of the new association, holotypus, is relevé 2 in Table 2. Relevé 13 in Table 2 is ecologically and floristically slightly different and shows certain similarity with the riparian forest from the association Lamio orvalae-Alnetum glutinosae (comp. Dakskobler 2016).

\section{Paeonio officinalis-Tiletum platyphylli}

Relevés in the third cluster in the dendrogram in Figure 1 are arranged in Table 3. We compared them with the stands of the subassociation Corydalido ochroleucaeAceretum pseudoplatani tilietosum platyphylli (Košir 2004, 8 relevés) and the stands of the association Paeonio officinalis-Tiletum platyphylli (Košir \& Surina 2005, 19 relevés). Based on the dendrogram in Figure 3 they can be 


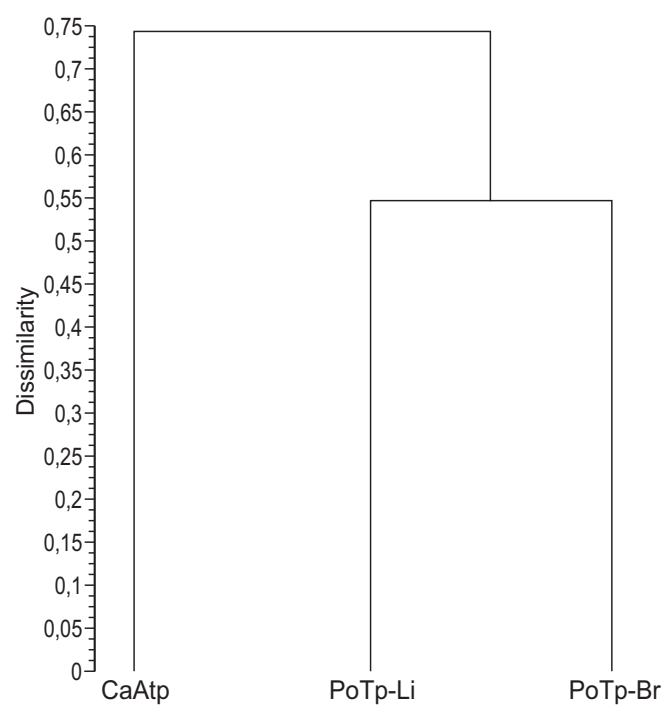

Figure 3: Dendrogram of the stands of the syntaxa Corydalido ochroleucae-Aceretum pseudoplatani tilietosum platyphylli (CaAtp), Paeonio officinalis-Tilietum platyphylli - Lipnik in Čičarija (PoTp-Li), and Paeonio officinalis-Tilietum platyphylli - Brkinski Rob and Gora under Železna Vrata (PoTp-Br) - UPGMA, 1-similarity ratio. Slika 3: Dendrogram sestojev sintaksonov Corydalido ochroleucaeAceretum pseudoplatani tilietosum platyphylli (CaAtp), Paeonio officinalis-Tilietum platyphylli - Lipnik v Čičariji (PoTp-Li) in Paeonio offcinalis-Tilietum platyphylli - Brkinski rob in Gora pod Železnimi vrati (PoTp-Br) - UPGMA, 1-similarity ratio

classified into the latter, although the character species of this association, namely Aristolochia lutea, Ornithogalum umbellatum and Smyrnium perfoliatum, were not recorded and Paeonia officinalis occurs in only one relevé. The only more frequent character species is Helleborus multifidus subsp. istriacus (H. odorus subsp. istriacus, $H$. istriacus) but this species is also frequent in the stands of the subassociation Corydalido ochroleucae-Aceretum pseudoplatani veratretosum nigri. Differential species of researched community against the stands of this subassociation should be also Sorbus aria (Aira edulis) and Euonymus verrucosa. Nevertheless, total floristic similarity between our stands and the stands of the association Paeonio officinalis-Tiletum platyphylli according to Sørensen (1948) is around 55\% and both have the large-leaved lime dominating in the tree layer, which allows us to classify them into the same association. We also identified another character species of the association - Cnidium silaifolium. We cannot, however, classify these stands into any of previously described subassociations tilietosum platyphylli or fraxinetosum excelsioris.

Relevés 1-8 are classified into the new subassociation Paeonio officinalis-Tilietum platyphylli saxifragetosum rotundifoliae subass. nov. hoc loco. Its nomenclatural type, holotypus, is relevé 2 in Table 3. Differential species of the subassociation include Pseudofumaria alba, Galeobdolon montanum and Euonymus verrucosa, and the stands indicate certain similarity also with the stands of the association Corydalido ochroleucae-Aceretum pseudoplatani. They are differentiated from the latter by thermophytic species like Cnidium silaifolium, Tanacetum corymbosum, Helleborus istriacus, Mercurialis ovata and Melittis melisophyllum. Relevés 9-13 in Table 3 are classified into the subassociation Paeonio officinalis-Tilietum platyphylli castaneetosum sativae. Its nomenclatural type, holotypus, is relevé 11 in Table 3. Differential species of the subassociation are Castanea sativa, Cornus mas, Ruscus aculeatus and Hedera helix. They characterise a more thermophilic, colline-submontane form of this association, which was found on the slopes of Gora under Železna Vrata at the elevation of only $310 \mathrm{~m}$ to $340 \mathrm{~m}$. Typically, the stands of this subassociation have a larger admixture of Ulmus glabra, which occasionally even dominates the tree layer.

Stands in the extreme left cluster in the dendrogram in Figure 2 were arranged in Table 4. Three relevés stand out with the tree layer that is dominated either by hop hornbeam (Seslerio autumnalis-Ostyretum), downy oak and Turkey oak (Seslerio autumnalis-Quercetum cerridis I Aristolochio luteae-Quercetum pubescentis?) or common hornbeam (Asaro-Carpinetum betuli). The first five relevés in this table can still be classified into the association Paeonio-Tilietum platyphylli, but into the new subassociation hierochloetosum australis. Its nomenclatural type, holotypus, is releve 3 in Table 4. The differential species of the subassociation are Hierochloë australis, Sesleria tenuifolia and Plagiomnium undulatum, but Sesleria autumnalis also has high mean cover. Differential against other subassociations are also some species of the classes Erico-Pinetea (Amelanchier ovalis, Chamaecytisus hirsutus), and TrifolioGeranietea (Anthericum ramosum, Digitalis grandiflora, Melampyrum velebiticum). This is also a distinctly thermophilic form of this association with localities in Orleška Draga, at elevations ranging between 280 and $340 \mathrm{~m}$.

\section{Lamio orvalae-Tilietum platyphylli}

We included another large-leaved lime community in the synthetic table comprising with five syntaxa described in this article (Table 6). This community is distinctly different from the other five. Its relevés were not made in the Karst or immediate vicinity, but in the Čepovan valley, mainly on shady (northwestern) slopes of the Trnovo Forest Plateau, at the contact of the sub-Mediterranean and Dinaric phytogeographical regions, in the montane belt at elevations ranging between $500 \mathrm{~m}$ and $700 \mathrm{~m}$. Most of these large-leaved lime stands (large and small-leaved lime are occasionally quite equal) are pioneer stands that developed on former agricultural areas, especially pastures, mainly on potential sites of the montane beech forest from the 
association Lamio orvalae-Fagetum. They cannot be classified into any of large-leaved lime associations previously described in Slovenia nor into the association Saxifrago petraeae-Tilietum platyphylli, whose stands occur on much more extreme and rocky sites (comp. Dakskobler 2007). We therefore classified it into the new association Lamio orvalae-Tilietum platyphylli ass. nov. hoc loco. Its nomenclatural type, holotypus, is relevé 4 in Table 5. The diagnostic species of the association are Tilia plytyphyllos, T. cordata, Lamium orvala, Anemone trifolia, Helleborus odorus, Sesleria autumnalis and Arum maculatum. The listed species characterise the new association both ecologically and chorologically, and indicate its syndynamic relationship with the stands of the association Lamio orvalae-Fagetum. We differentiate between two variants, a slightly more thermophilic (littoral) variant with Sesleria autumnalis (relevés 1-9 in Table 5, its differential species include Adoxa moschatellina), and a slightly more frigoriphilic (Dinaric) variant with Omphalodes verna (relevés 10-13 in Table 5).

Synthetic table 6 also demonstrates that this is probably a secondary large-leaved lime community, which differs from other communities described herein in particular with a higher proportion of the species of the alliance Aremonio-Fagion, order Fagetalia sylvaticae and class Querco-Fagetea.

\section{Conspectus of the syntaxa described herein}

The younger of us (ID) prefer conservative view:

Querco-Fagetea Br.-Bl. \& Vlieg. 1937 (Carpino-Fagetea Passarge et Hofmann 1968)

Fagetalia sylvaticae Walas 1933

Tilio-Acerion Klika 1955 (also possible Fraxino excelsioris-Acerion pseudoplatani P. Fukarek 1969)

Ostryo-Tilienion platyphylli P. Košir, Čarni et $\mathrm{Di}$ Pietro 2008

\section{Corydalido ochroleucae-Aceretum pseudoplatani}

Accetto 1991

veratretosum nigri Dakskobler et Reščič 2015

var. Fraxinus excelsior var. nov.

var. Isopyrum thalictroides var. nov.

Paeonio officinalis-Tiletum platyphylli P. Košir et

Surina 2005

saxifagetosum rotundifoliae subass. nov.

castaneetosum sativae subass. nov.

hierochloetosum australis subass. nov.

Fraxino orni-Aceretum pseudoplatani ass. nov.

Lamio orvalae-Tilietum platyphylli ass. nov.

var. Sesleria autumnalis

var. Omphalode verna
The older of the authors (LP) prefers a more recent view, which partly corresponds also with Mucina et al. (2016).

Carpino-Fagetea sylvaticae Jakucs ex Passarge 1968

Aceretalia pseudoplatani Moor 1976 nom. conserv. propos. Fraxino excelsioris-Acerion pseudoplatani P. Fukarek 1969

or

Ostryo carpinifoliae-Tilion platyphylli (Košir et al. 2008)

Čarni in Willner et al. 2016

But the problem with classifying our communities into one of these two alliances is that the researched stands are in fact Submediterranean, not only thermophilic, but also mesophilic, and definitely not xero-thermophilic.

The conservative solution therefore better corresponds to the site conditions of the researched stands.

\section{Nature conservation function of the described noble hardwood communities in SW Slovenia}

The forests described herein belong to the habitat type of Community interest $9180^{*}$ Tilio-Acerion forests of slopes, screes and ravines. Forests in Orleška Draga, Risnik, Gora under Železna Vrata and Petnik gorge are part of the Natura 2000 Karst area. In terms of forest typology they belong to the forest site type of submontane-montane large-leaved lime stands on carbonate and mixed bedrock, for which we previously concluded (Dakskobler et al. 2013: 25) that they usually cover small areas, which can make their sites more vulnerable to large-scale interventions into forest. Forest management activities like tree felling and wood harvesting should be adapted to protecting their sites. Given the specifics of these sites and sensitivity of forest soils any construction of forest roads in their vicinity should be carefully planned.

The described forest stands also comprise sites of several protected species (Anon. 2004): Convallaria majalis, Cyclamen purpurascens, Dactlylorhiza fuchsii, Epipactis helleborine, Erythronium dens-canis, Galanthus nivalis, Helleborus odorus, $H$. multifidus subsp. istriacus, $H$. niger, Lilium bulbiferum, L. carniolicum, L. martagon, Listera ovata, Neottia nidus-avis, Orchis pallens, Orchis mascula subsp. speciosa, Paeonia officinalis, P. mascula agg. (P. daurica), Primula auricula, Ruscus aculeatus and Sedum maximum as well as several Red List species (Anon. 2002): Muscari botryoides, Veratrum nigrum, endemic species Tephroseris pseudocrispa and species that is very rare in Slovenia ( $\mathrm{Hi}$ eracium lasiophyllum).

Collapse dolines Risnik and Orleška Draga are valuable natural features and botanical curiosities with distinct 
vegetation that is clearly different from the vegetation in their vicinity. They are home to numerous species characteristic for beech forests (order Fagetalia sylvaticae) and it is likely that beech once occurred here as well, perhaps even in ice-age refuges.

\section{Conclusions}

Noble hardwood communities are very rare in southwestern Slovenia and most of them are classified into the suballiance Ostryo-Tilienion platyphylli and associations Corydalido ochroleucae-Aceretum pseudoplatani and Paeonio officinalis-Tilietum platyphylli. Stands of associations Veratro nigri-Fraxinetum excelsioris and Saxifrago petraeaeTilietum platyphylli were found only in fragments. With our research we determined the occurrence of the stands of the association Corydalido ochroleucae-Aceretum also in two notable Karst collapse dolines, Risnik at Divača and Orleška Draga at Sežana. They differ from the stands in Čičarija and on Vremščica with slightly smaller dominance of sycamore and large-leaved lime and a higher proportion (cover) of small-leaved lime and common hornbeam in the tree layer, as well with certain more thermophilic species. The stands in Orleška Draga are classified as the new variant with Isopyrum thalictroides of the previously described subassociation veratretosum nigri with stands in Risnik. In Orleška Draga we observed a direct contact with a slightly similar common hornbeam community (Asaro-Carpinetum betuli lamietosum orvalae). The floristic composition of the association Paeonio officinalis-Tilietum platyphylli from the area of the original (first) description under Lipnik above Rakitovec is very different from the stands described under Brkinski Rob above Ratečevo Brdo. Nevertheless, these stands, which also share several species with the stands of the association Corydalido ochroleucae-Aceretum, can still be classified into this association as a new subassociation saxifragetosum rotundifoliae. We found two, even more marginal forms of the association Paeonio-Tilietum, slightly similar to the stands of the subassociation Seslerio autumnalis-Ostryetum tilietosum platyphylli, under Gora under Železna Vrata on the northern edge of the Karst and in Orleška Draga. The former can be classified into the new subassociation castaneetosum sativae and the latter into the new subassociation hierochloetosum australis.

At least in western Slovenia, large and small-leaved lime are common pioneers also on abandoned agricultural areas on beech forest sites. A higher proportion of species characteristic for beech and beech-oak forests indicates that some of the large-leaved lime stands in the Čepovan valley (at the contact between sub-Mediterranean and Dinaric phytogeographical regions) are secondary. Because their species composition is clearly different from previously described large-leaved lime communities they are classified into the new association Lamio orvalae-Tilietum platyphylli and treated as a pioneer stage on the sites of the association Lamio orvalae-Fagetum. The new association belongs to the group of secondary noble hardwood communities on beech or oak sites, which in the Soča Valley comprise also the association Ornithogalo pyrenaici-Fraxinetum excelsioris and occasionally (in the Karst) also Veronico sublobatae-Fraxinetum excelsioris.

Sycamore is the name-giving species for predominantly montane communities of noble hardwoods. Sycamore and wych elm stands in the colline belt (at elevations of not more than 150-235 m) in the deep gorge of Petnik on the northern edge of the Karst, at the contact of flysch and limestone, cannot be classified into the association Veratro nigri-Fraxinetum excelsioris which is known in this belt elsewhere in the Primorska region, due to complete absence of ash in its stands. Similarly, they cannot be classified into the association Lamio orvalae-Aceretum pseudoplatani, even though Acer pseudoplatanus and Lamium orvala both dominate these stands, each in its layer, because this name has already been used for the altimontane community of noble hardwoods in the SE Alps and the Dinaric Alps from the suballiance Lunario-Acerenion. We therefore described a new association Fraxino orni-Aceretum pseudoplatani, which is characteristic for the northern edge of the Karst.

Although noble hardwood stands in SW Slovenia cover very small areas they are a Natura 2000 habitat type that deserves special attention, protection and proper management.

\section{Povzetek}

Fitocenološka analiza gozdnih sestojev plemenitih listavcev (Ostryo-Tilienion platyphylli) na Krasu in njegovih robnih območjih

Združbe plemenitih listavcev so v jugozahodni Sloveniji precejšnja redkost in $\mathrm{v}$ glavnem jih uvrščamo $\mathrm{v}$ podzvezo Ostryo-Tilienion platyphylli in v asociaciji Corydalido ochroleucae-Aceretum pseudoplatani in Paeonio officinalis-Tilietum platyphylli, le v sledovih smo našli sestoje asociacij Veratro nigri-Fraxinetum excelsioris in Saxifrago petraeae-Tilietum platyphylli. Z našimi raziskavami smo ugotovili uspevanje sestojev asociacije Corydalido ochroleucae-Aceretum tudi v dveh znamenitih kraških udornicah, Risniku pri Divači in Orleški dragi pri Sežani. Od sestojev v Čičariji in na Vremščici se razlikujejo po nekoliko manjši prevladi gorskega javorja in lipe in po večjem deležu (zastiranju) lipovca in belega gabra $\mathrm{v} v$ drevesni plasti, 
prav tako po nekaterih bolj toploljubnih vrstah. Sestojem v Risniku že prej opisane subasociacije veratretosum nigri lahko priključimo tudi sestoje nove variante $\mathrm{z}$ vrsto Isopyrum thalictroides v Orleški dragi. Tam opažamo neposreden stik z nekoliko podobno združbo belega gabra (Asaro-Carpinetum betuli lamietosum orvalae). Floristična sestava asociacije Paeonio officinalis-Tilietum platyphylli iz območja izvornega opisa pod Lipnikom nad Rakitovcem je precej drugačna od sestojev, ki smo jih popisali pod Brkinskim robom nad Ratečevim Brdom, a kljub temu lahko te sestoje, ki imajo sicr tudi nekatere skupne vrste s sestoji asociacije Corydalido ochroleucae-Aceretum, vseeno še lahko uvrstimo v to asociacijo, kot novo subasociacijo saxifragetosum rotundifoliae. Še bolj robni obliki asociacije Paeonio-Tilietum, nekoliko podobni sestojem subasociacije Seslerio autumnalis-Ostryetum tilietosum platyphylli smo našli pod Goro pod Železnimi vrati na severnem robu Krasa in v Orleški dragi. Prve lahko uvrstimo v novo subasociacijo castaneetosum sativae, druge prav tako $\mathrm{v}$ novo subasociacijo hierochloetosum australis.

Lipa in lipovec sta vsaj v zahodni Sloveniji pogosta pionirja tudi na opuščenih kmetijskih površinah na rastiščih bukovih gozdov. Na drugotnost nekaterih lipovih sestojev v Čepovanski dolini (na stiku med submediteranskim in dinarskim fitogeografskim območjem) kaže povečan delež vrst značilnih za bukove in bukovo-hrastove gozdove. Ker so ti sestoji po vrstni sestavi očitno drugačni od prej omenjenih lipovih združb, jih uvrščamo v novo asociacijo Lamio orvalae-Tilietum platyphylli in jih obravnavamo kot pionirski stadij na rastiščih asociacije Lamio orvalae-Fagetum. Nova asociacija sodi v skupino drugotnih združb plemenitih listavcev na bukovih ali hrastovih rastiščih, v kateri sta tudi asociaciji Ornithogalo pyrenaici-Fraxinetum excelsioris $\mathrm{v}$ Posočju in Veronico sublobatae-Fraxinetum excelsioris ponekod na Krasu.

Po gorskem javorju se v glavnem imenujejo montanske združbe plemenitih listavcev. Sestojev gorskega javorja in gorskega bresta $\mathrm{v}$ gričevnatem pasu (nadmorska višina le $150 \mathrm{~m}$ do $235 \mathrm{~m}$ ) v globoki grapi Petnik na severnem robu Krasa, na stiku fliša in apnenca, ne moremo uvrstiti $\mathrm{v}$ asociacijo Veratro nigri-Fraxinetum excelsioris, ki jo sicer poznamo v tem višinskem pasu drugod na Primorskem, ker v njenih sestojih velikega jesena sploh nismo popisali. Prav tako jih ne moremo uvrstiti v asociacijo Lamio orvalae-Aceretum pseudoplatani, čeprav sta vrsti Acer pseudoplatanus in Lamium orvala dejansko v njih pravladujoči, vsaka v svoji plasti. To ime je namreč že uporabljeno za altimontansko združbo plemenitih listavcev v Jugovzhodnih Alpah in Dinarskem gorstvu iz podzveze LunarioAcerenion. Iz teh razlogov smo opisali novo asociacijo Fraxino orni-Aceretum pseudoplatani in je posebnost severnega roba Krasa.
Čeprav so sestoji plemenitih listavcev v jugozahodni Sloveniji razširjeni na zelo majhnih površinah, so kot Natura 2000 habitatni tip vredni pozornosti, varovanja in ustreznega gopodarjenja.

\section{Acknowledgements}

The authors acknowledge the financial supportd from the Slovenian Research Agency and the Ministry of Agriculture, Forestry and Food (research core funding No. P10236, target research The design of monitoring of the conservation status of minor Natura 2000 forest habitat types in Slovenia, V4-1430). Sincere thanks to Matej Reščič for his help in the field work, to Doc. Dr. Andrej Rozman for telling us about Prof. Gabrijel Tomažičs manuscript table, to Prof. Dr. Jean-Paul Theurillat for his valuable data about the association Rusco aculeati-Aceretum pseudoplatani and to Prof. Dr. Andraž Čarni and Dr. Marisa Vidali for their suggestions. Two anonymous reviewers helped us with valuable improvements and corrections. English translation by Andreja Šalamon Verbič.

\section{References}

Accetto, M. 1991: Corydalido ochroleucae-Aceretum ass. nova v Sloveniji. Razprave 4. razreda SAZU 32 (3): 89-128.

Braun-Blanquet, J., 1964: Pflanzensoziologie. 3. Auflage. Grundzüge der Vegetations Kunde, Springer Verlag, Wien, New York, 865 pp.

Buser, S., 2009: Geološka karta Slovenije 1: 250.000. Geological map of Slovenia 1: 250,000. Geološki zavod Slovenije, Ljubljana.

Cegnar T., 1998: Temperatura zraka. In: Fridl, J., D. Kladnik, M. Orožen Adamič \& D. Perko: Geografski atlas Slovenije. Država v prostoru in času. Državna založba Slovenije, Ljubljana, pp. 100-101.

Dakskobler, I. 2006: Prispevek k poznavanju gozdne vegetacije Krasa (jugozahodna Slovenija). Annales, Ser. hist. nat. 16 (1): 57-76.

Dakskobler, I. 2007: Gozdovi plemenitih listavcev v Posočju. Forest of valuable broad-leaved tree species in the Soča valley (western Slovenia). Scopolia 60: 1-287.

Dakskobler, I. 2016: Phytosociological analysis of riverine forests in the Vipava and Reka Valleys (southwestern Slovenia). Folia biologica et geologica 57 (1): 5-61.

Dakskobler, I. \& Reščič, M. 2015: Fitocenološka in

gozdnogospodarska analiza gorskega bukovega in javorovega gozda na skalnatih rastiščih na Krasu in v Čičariji (JZ Slovenija). Gozdarski vestnik 73 (2): 67-87.

Dakskobler, I., Košir, P. \& Kutnar, L. 2013: Gozdovi plemenitih listavcev v Sloveniji. Združbe gorskega javorja, gorskega bresta, velikega jesena, ostrolistnega javorja, lipe in lipovca. Silva Slovenica in Zveza gozdarskih društev, Ljubljana, $74 \mathrm{pp}$. 
Géhu, J.-M. 1997: Observations phytosociologiques préliminaires sur le littoral occidental de l' Íle de Jersey (Anglo-Normande). Colloques Phytosociologiques 27: 169-196.

Košir, P. \& Surina, B. 2005: Paeonio officinalis-Tilietum platyphylli - nova združba gozdov plemenitih listavcev v Čičariji (jugozahodna Slovenija). In: Rožac Darovec, V. (ed.): Meje in konfini. Koper, Univerza na Primorskem, Znanstveno-raziskovalno središče Koper, Založba Annales : Zgodovinsko društvo za južno Primorsko, pp. 345-366.

Košir, P. 2004: Sinsistematski pregled šuma plemenitih listača ilirske florne provincije s posebnim osvrtom na zajednice u Sloveniji. Doktorska disertacija. Sveučilište u Zagrebu, Šumarski fakultet, Zagreb, 164 pp. + priloge.

Košir, P. 2005: Noble hardwood forests of the altimontane belt (Lamio orvalae-Aceretum pseudoplatani P. Košir et Marinček 1999) in Slovenia (western part of the Illyrian floral province). Natura Croatica 14 (2): 59-86.

Košir, P., Čarni, A. \& di Pietro, R. 2008: Classification and phytogeographical differentiation of broad-leaved ravine forests in southeastern Europe. Journal of Vegetation Science 19 (3): 331-342. DOI: https://doi.org/10.3170/2008-8-18372

Maarel van der, E. 1979: Transformation of cover-abundance values in phytosociology and its effects on community similarity. Vegetatio 39 (2): 97-114. DOI: https://doi.org/10.1007/BF00052021

Martinčič, A. 2003: Seznam listnatih mahov (Bryopsida) Slovenije. Hacquetia 2 (1): 91-166.

Martinčič, A. 2011: Seznam jetrenjakov (Marchanthiophyta) in rogovnjakov (Anthocerotophyta) Slovenije. Scopolia 72: 1-38.

Martinčič, A., Wraber, T. Jogan, N. Podobnik, A., Turk, B., Vreš, B., Ravnik, V., Frajman, B., Strgulc Krajšek, S., Trčak, B., Bačič, T., Fischer, M. A., Eler, K. \& Surina, B. 2007: Mala flora Slovenije. Ključ za določanje praprotnic in semenk. Četrta, dopolnjena in spremenjena izdaja. Tehniška založba Slovenije, Ljubljana. 967 pp.

Mucina, L., Bultmann, H., Dierssen, K., Theurillat, J.-P., Raus, T., Čarni, A., Šumberová, K., Willner, W., Dengler, J., Garcia, R. G., Chytrý, M., Hájek, M., Di Pietro, R., Iakushenko, D., Pallas, J., Daniëls, F. J. A., Bergmeier, E., Santos Guerra, A., Ermakov, N., Valachovič, M., Schaminée, J. H. J., Lysenko, T., Didukh, Y. P., Pignatti, S., Rodwell, J. S., Capelo, J., Weber, H. E., Solomeshch, A., Dimopoulos, P., Aguiar, C., Hennekens, S. M. \& Tichý, L. 2016: Vegetation of Europe: hierarchical floristic classification system of vascular plant, bryophyte, lichen, and algal communities. Applied Vegetation Science 19: 3-264. DOI: https://doi.org/10.1111/ avsc. 12257
Ogrin, D. 1996: Podnebni tipi v Sloveniji. Geografski vestnik 68: $39-56$.

Peruzzi L. 2016: Crocus heuffelianus (Iridaceae), a new record for the Italian flora. Phytotaxa, 261: 291-294. DOI: https://doi. org/10.11646/phytotaxa.261.3.10

Podani, J. 2001: SYN-TAX 2000. Computer Programs for Data Analysis in Ecology and Systematics. User's Manual, Budapest, 53 pp.

Poldini, L. 1985: L’ Asaro-Carpinetum betuli Lausi 64 del Carso Nordadriatico. Studia Geobotanica 5: 31-38.

Poldini, L. 2009: La diversità vegetale del Carso fra Trieste e Gorizia. Lo stato dell'ambiente. Edizione Goliardiche, Trieste, 732 pp.

Rottensteiner, W. K. 2016: Attempt of a morphological differentiation of Helleborus species in the Northwestern Balkans. Modern Phytomorphology 9 (Suppl.): 17-33.

Seliškar, T., Vreš, B. \& Seliškar, A. 2003: FloVegSi 2.0. Računalniški program za urejanje in analizo bioloških podatkov. Biološki inštitut ZRC SAZU, Ljubljana.

Sørensen, Th. 1948: A method of establishing groups of equal amplitude in plant sociology based on similarity of species content. Det Kongelige Danske Videnskaberns Selskab, Biologiske Skrifter 5 (4): $1-34$.

Suppan, U., Prügger, J. \& Mayrhofer, H. 2000: Catalogue of the lichenized and lichenicolous fungi of Slovenia. Bibliotheca Lichenologica 76: 1-215.

Šilc, U. \& Čarni, A. 2012: Conspectus of vegetation syntaxa in Slovenia. Hacquetia 11 (1): 113-164. DOI: https://doi.org/10.2478/ v10028-012-0006-1

Šilc, U., Čarni, A., Dakskobler, I., Kutnar, L., Marinšek, A., Rozman, A., Sajko, I. \& Vreš, B. 2017: Zasnova monitoringa stanja ohranjenosti manjšinskih Natura 2000 gozdnih habitatnih tipov v Sloveniji : zaključno poročilo v okviru Ciljnega raziskovalnega projekta (V41430). Elaborat. Biološki inštitut ZRC SAZU, Ljubljana, 170 pp.

Urbančič, M., Simončič, P., Prus, T. \& Kutnar, L. 2005: Atlas gozdnih tal. Zveza gozdarskih društev Slovenije, Gozdarski vestnik \& Gozdarski inštitut Slovenije, Ljubljana, 100 pp.

Zupančič, B. 1998: Padavine. In: Orožen Adamič, M., Fridl, J., Kladnik, D. \& Perko, D. (eds.): Geografski atlas Slovenije. Država v prostoru in času. Državna založba Slovenije, Ljubljana, pp. 98-99. 
Table 1 (Tabela 1): Corydalido ochroleucae-Aceretum pseudoplatani Risnik, Orleška Draga.

\begin{tabular}{|c|c|c|c|c|c|c|c|c|c|c|c|c|c|c|c|c|}
\hline & \multicolumn{2}{|l|}{ Number of relevé (Zaporedna številka popisa) } & 1 & 2 & 3 & 4 & 5 & 6 & 7 & 8 & 9 & 10 & 11 & 12 & Pr. & Fr. \\
\hline & \multicolumn{2}{|l|}{$\begin{array}{l}\text { Database number of relevé } \\
\text { (Delovna številka popisa) }\end{array}$} & 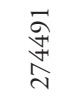 & 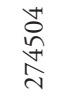 & 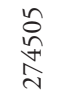 & 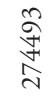 & 离 & 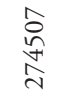 & 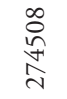 & $\underset{\substack{c \\
\stackrel{4}{2}}}{\stackrel{4}{a}}$ & 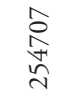 & 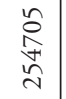 & 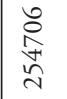 & $\begin{array}{l}\vec{\sim} \\
\underset{+}{\sim} \\
\stackrel{\sim}{*}\end{array}$ & & \\
\hline & \multicolumn{2}{|l|}{ Elevation in $\mathrm{m}$ (Nadmorska višina $\mathrm{v} \mathrm{m})$} & 300 & 320 & 330 & 300 & 280 & 265 & 275 & 370 & 370 & 375 & 370 & 275 & & \\
\hline & \multicolumn{2}{|l|}{ Aspect (Lega) } & NNE & NW & NW & $\mathrm{N}$ & NW & $\mathrm{N}$ & SEE & $\mathrm{NE}$ & W & SE & NW & $\mathrm{W}$ & & \\
\hline & \multicolumn{2}{|l|}{ Slope in degrees (Nagib v stopinjah) } & 25 & 25 & 30 & 25 & 25 & 20 & 25 & 25 & 25 & 15 & 10 & 25 & & \\
\hline & \multicolumn{2}{|l|}{ Parent material (Matična podlaga) } & Gr & $\mathrm{Gr}$ & $\mathrm{Gr}$ & $\mathrm{Gr}$ & Gr & $\mathrm{Gr}$ & Gr & A & Gr & A & A & A & & \\
\hline & \multicolumn{2}{|l|}{ Soil (Tla) } & $\operatorname{Re}$ & $\operatorname{Re}$ & $\operatorname{Re}$ & $\operatorname{Re}$ & $\operatorname{Re}$ & $\operatorname{Re}$ & Co & $\operatorname{Re}$ & $\operatorname{Re}$ & $\operatorname{Re}$ & $\mathrm{Rj}$ & $\mathrm{Rj}$ & & \\
\hline & \multicolumn{2}{|l|}{ Stoniness in \% (Kamnitost v \%) } & 20 & 20 & 30 & 10 & 10 & 30 & 10 & 90 & 60 & 80 & 10 & 10 & & \\
\hline & \multicolumn{16}{|l|}{ Cover in \% (Zastiranje v \%): } \\
\hline & Upper tree layer (Zgornja drevesna plast) & E3b & 80 & 80 & 80 & 80 & 90 & 80 & 80 & 90 & 90 & 80 & 90 & 85 & & \\
\hline & Lower tree layer (Spodnja drevesna plasti) & E3a & 10 & 10 & 10 & 19 & 10 & 5 & 10 & . & . & 10 & . & . & & \\
\hline & Shrub layer (Grmovna plast) & E2 & 15 & 10 & 80 & 19 & 5 & 10 & 10 & 10 & 60 & 10 & 1 & 5 & & \\
\hline & Herb layer (Zeliščna plast) & E1 & 80 & 90 & 30 & 80 & 90 & 90 & 80 & 60 & 80 & 60 & 70 & 90 & & \\
\hline & Moss layer (Mahovna plast) & E0 & 30 & 10 & & 10 & 10 & 30 & 20 & 60 & 30 & 70 & 5 & 10 & & \\
\hline & Max. tree diameter (Maks. premer dreves) & $\mathrm{cm}$ & 45 & 40 & 40 & 35 & 40 & 35 & 35 & 30 & 30 & 25 & 30 & 30 & & \\
\hline & Max. tree height (Maks. višina dreves) & $\mathrm{m}$ & 24 & 20 & 18 & 20 & 22 & 20 & 18 & 17 & 17 & 17 & 20 & 20 & & \\
\hline & Number of species (Število vrst) & & 60 & 51 & 56 & 53 & 51 & 41 & 47 & 80 & 65 & 53 & 46 & 30 & & \\
\hline & Relevé area (Velikost popisne ploskve) & $\mathrm{m}^{2}$ & 400 & 400 & 400 & 400 & 400 & 400 & 400 & 400 & 400 & 400 & 400 & 400 & & \\
\hline & \multicolumn{2}{|l|}{ Date of taking relevé (Datum popisa) } & 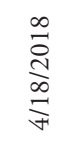 & 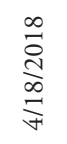 & $\underset{\underset{\sim}{\infty}}{\stackrel{\infty}{\leftrightarrows}}$ & 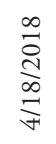 & 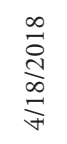 & 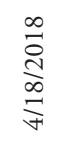 & 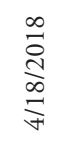 & 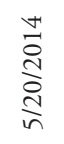 & 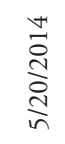 & 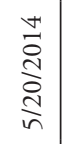 & 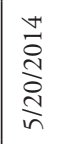 & 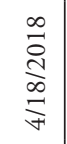 & & \\
\hline & \multicolumn{2}{|l|}{ Locality (Nahajališče) } & 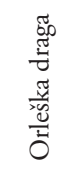 & 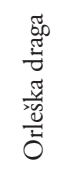 & 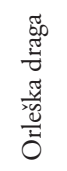 & 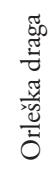 & 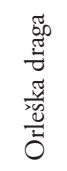 & 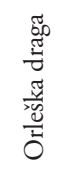 & 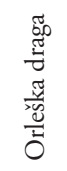 & $\frac{y}{\vec{a}}$ & 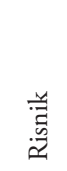 & 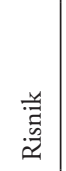 & 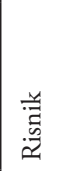 & 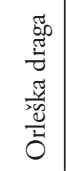 & & \\
\hline & \multicolumn{2}{|l|}{ Quadrant (Kvadrant) } & 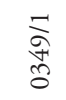 & 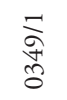 & 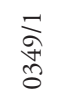 & 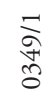 & 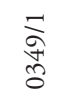 & 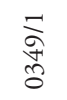 & 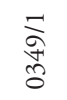 & 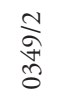 & 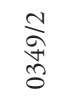 & 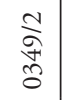 & 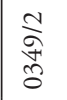 & 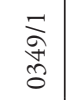 & & \\
\hline & Coordinate (Koordinate) GK Y (D-48) & $\mathrm{m}$ & \begin{tabular}{l}
$\infty$ \\
$\stackrel{\sim}{+}$ \\
$\stackrel{+}{+}$ \\
\multirow{+}{+}{}
\end{tabular} & 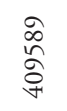 & 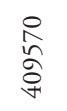 & 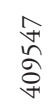 & $\begin{array}{l}\tilde{n} \\
\hat{\sigma} \\
\hat{\sigma}\end{array}$ & $\begin{array}{l}\bar{n} \\
\stackrel{5}{\circ} \\
\stackrel{+}{+}\end{array}$ & 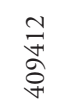 & $\begin{array}{l}\stackrel{+}{\infty} \\
\stackrel{+}{F}\end{array}$ & $\begin{array}{l}\underset{\widetilde{Z}}{\sigma} \\
\stackrel{\sigma}{\sigma}\end{array}$ & $\begin{array}{l}\text { ते } \\
\infty \\
\stackrel{+}{\forall}\end{array}$ & $\begin{array}{l}\text { ర్ } \\
\infty \\
\stackrel{+}{\forall}\end{array}$ & 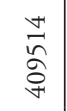 & & \\
\hline & Coordinate (Koordinate) GK X (D-48) & $\mathrm{m}$ & 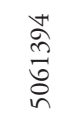 & 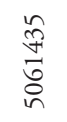 & $\begin{array}{l}\stackrel{+}{ } \\
\stackrel{+}{ \pm} \\
\stackrel{\circ}{\circ}\end{array}$ & 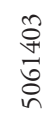 & $\begin{array}{l}\infty \\
\stackrel{\infty}{+} \\
\stackrel{\circ}{\circ}\end{array}$ & 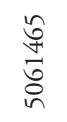 & $\begin{array}{l}\stackrel{a}{\vec{t}} \\
\stackrel{\circ}{n}\end{array}$ & $\begin{array}{l}o \\
\curvearrowleft \\
\hat{n} \\
\hat{n} \\
i n\end{array}$ & 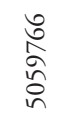 & 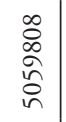 & 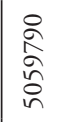 & $\begin{array}{l}\stackrel{+}{0} \\
\stackrel{n}{n} \\
\stackrel{0}{\circ} \\
\stackrel{n}{n}\end{array}$ & & \\
\hline & \multicolumn{12}{|c|}{ Diagnostic species of the association (Diagnostična vrsta asociacije) } & & & & $\dot{4} \frac{0}{1}$ \\
\hline $\mathrm{CF}$ & Pseudofumaria alba & E1 & . & . & . & $\cdot$ & . & . & . & + & + & 1 & . & . & 3 & 30 \\
\hline & \multicolumn{12}{|c|}{ Differential species of the subassociation (Razlikovalnice subasociacije) } & & & & \\
\hline QF & Veratrum nigrum & E1 & 2 & . & $\mathrm{r}$ & 2 & + & 1 & 1 & 1 & 1 & 1 & . & + & 9 & 90 \\
\hline QF & Viola mirabilis & E1 & + & . & . & + & . & . & + & 1 & + & + & . & . & 6 & 60 \\
\hline \multirow[t]{2}{*}{ TG } & Campanula rapunculoides & E1 & . & 1 & . & + & + & . & + & + & + & . & . & . & 6 & 60 \\
\hline & \multicolumn{9}{|c|}{ Diferential species of the variants (Razlikovalnice variant) } & & & & & & & \\
\hline TA & Isopyrum thalictroides & E1 & 2 & 2 & 3 & 2 & 1 & 2 & 1 & . & . & . & . & + & 7 & 70 \\
\hline FS & Viola reichenbachiana & E1 & 1 & 1 & + & 1 & 1 & . & + & . & . & . & + & + & 6 & 60 \\
\hline QP & Mercurialis ovata & E1 & + & + & + & + & . & + & 1 & . & . & . & . & . & 6 & 60 \\
\hline QF & Acer campestre & E3b & . & . & . & + & + & 1 & . & . & + & . & . & + & 4 & 40 \\
\hline QF & Acer campestre & E3a & + & 1 & + & + & + & + & . & . & . & . & . & . & 6 & 60 \\
\hline QF & Acer campestre & $\mathrm{E} 2 \mathrm{~b}$ & . & + & + & . & . & . & . & . & . & . & . & + & 2 & 20 \\
\hline QF & Acer campestre & $\mathrm{E} 2 \mathrm{a}$ & . & + & . & . & . & . & . & . & . & . & . & . & 1 & 10 \\
\hline QF & Melica uniflora & E1 & + & + & + & + & + & . & . & . & . & . & . & . & 5 & 50 \\
\hline
\end{tabular}




\begin{tabular}{|c|c|c|c|c|c|c|c|c|c|c|c|c|c|c|c|c|}
\hline & Number of relevé (Zaporedna številka popisa) & & 1 & 2 & 3 & 4 & 5 & 6 & 7 & 8 & 9 & 10 & 11 & 12 & Pr. & Fr. \\
\hline $\mathrm{MuA}$ & Aconitum lycoctonum & E1 & . & . & . & . & . & . & . & 3 & 3 & + & + & . & 3 & 30 \\
\hline $\mathrm{AF}$ & Hacquetia epipactis & E1 & . & . & . & . & . & . & . & + & + & + & 2 & . & 3 & 30 \\
\hline FS & Fraxinus excelsior & $\mathrm{E} 3 \mathrm{~b}$ & . & . & . & . & . & . & . & + & . & . & . & . & 1 & 10 \\
\hline FS & Fraxinus excelsior & $\mathrm{E} 2 \mathrm{a}$ & . & . & . & . & $\cdot$ & . & . & + & + & $\mathrm{r}$ & . & . & 3 & 30 \\
\hline FS & Fraxinus excelsior & E1 & . & . & . & . & . & . & . & . & . & . & $\mathrm{r}$ & . & . & ${ }^{\circ}$ \\
\hline \multirow[t]{29}{*}{ TA } & Tilio-Acerion & & & & & & & & & & & & & & & \\
\hline & Acer pseudoplatanus & $\mathrm{E} 3 \mathrm{~b}$ & 2 & . & + & 2 & 2 & 2 & + & 3 & 4 & 4 & 1 & . & 9 & 90 \\
\hline & Acer pseudoplatanus & E3a & . & . & . & . & $\cdot$ & . & . & + & . & . & . & . & 1 & 10 \\
\hline & Acer pseudoplatanus & $\mathrm{E} 2 \mathrm{~b}$ & . & + & . & . & . & . & . & . & . & . & . & . & 1 & 10 \\
\hline & Acer pseudoplatanus & $\mathrm{E} 2 \mathrm{a}$ & . & + & . & . & + & . & . & . & . & . & . & . & 2 & 20 \\
\hline & Acer pseudoplatanus & E1 & . & 1 & 1 & 1 & 1 & . & 1 & . & + & 1 & 1 & 1 & 7 & 70 \\
\hline & Tilia cordata & $\mathrm{E} 3 \mathrm{~b}$ & 1 & 2 & 2 & 2 & 2 & + & 2 & 1 & + & . & . & + & 9 & 90 \\
\hline & Tilia cordata & E3a & + & 1 & . & . & . & + & . & . & . & . & . & + & 3 & 30 \\
\hline & Tilia cordata & $\mathrm{E} 2 \mathrm{a}$ & + & . & . & . & . & . & . & . & . & . & . & . & 1 & 10 \\
\hline & Tilia platyphyllos & $\mathrm{E} 3 \mathrm{~b}$ & 3 & + & 1 & 1 & + & + & . & 3 & 3 & 1 & . & . & 9 & 90 \\
\hline & Tilia platyphyllos & E3a & 1 & . & . & . & . & . & . & + & . & . & . & . & 2 & 20 \\
\hline & Tilia platyphyllos & $\mathrm{E} 2 \mathrm{~b}$ & . & . & . & . & . & . & . & . & + & . & . & . & 1 & 10 \\
\hline & Tilia platyphyllos & $\mathrm{E} 2 \mathrm{a}$ & 1 & . & . & . & . & . & . & . & . & . & . & . & 1 & 10 \\
\hline & Adoxa moschatellina & E1 & 1 & . & + & + & + & 1 & 1 & 1 & + & 1 & 1 & + & 9 & 90 \\
\hline & Geranium robertianum & E1 & 1 & . & + & . & . & + & . & + & 1 & + & . & . & 6 & 60 \\
\hline & Ulmus glabra & $\mathrm{E} 3 \mathrm{~b}$ & . & 1 & 2 & . & . & . & . & . & . & . & . & . & 2 & 20 \\
\hline & Ulmus glabra & $\mathrm{E} 3 \mathrm{a}$ & + & 1 & + & + & . & . & . & + & . & + & . & . & 6 & 60 \\
\hline & Ulmus glabra & $\mathrm{E} 2 \mathrm{a}$ & . & . & + & . & . & . & + & + & + & + & . & . & 5 & 50 \\
\hline & Ulmus glabra & E1 & . & . & . & + & + & . & + & . & . & . & . & . & 3 & 30 \\
\hline & Aruncus dioicus & E1 & 2 & + & . & 2 & + & . & . & 1 & . & + & . & . & 6 & 60 \\
\hline & Corydalis solida & E1 & 1 & . & + & . & + & + & . & 1 & . & . & . & + & 5 & 50 \\
\hline & Phyllitis scolopendrium & E1 & . & . & . & 1 & + & . & . & + & + & . & . & . & 4 & 40 \\
\hline & Thalictrum aquilegiifolium & E1 & + & . & . & . & . & . & . & + & . & + & . & . & 3 & 30 \\
\hline & Acer platanoides & E3b & + & . & . & . & 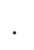 & . & . & 1 & . & . & . & . & 2 & 20 \\
\hline & Acer platanoides & E3a & . & . & . & . & . & . & . & + & . & . & . & . & 1 & 10 \\
\hline & Acer platanoides & $\mathrm{E} 2 \mathrm{a}$ & 1 & . & . & . & . & . & + & . & . & . & . & . & 2 & 20 \\
\hline & Acer platanoides & E1 & 1 & . & . & . & . & . & . & + & . & . & + & . & 2 & 20 \\
\hline & Aesculus hippocastanum & E3a & . & . & . & . & + & . & . & . & . & . & . & . & 1 & 10 \\
\hline & Juglans regia & $\mathrm{E} 2 \mathrm{a}$ & . & . & . & . & $\cdot$ & . & . & + & . & . & . & . & 1 & 10 \\
\hline \multirow[t]{3}{*}{$\mathrm{EC}$} & Erythronio-Carpinion & & & & & & & & & & & & & & & \\
\hline & Galanthus nivalis & E1 & 2 & 2 & 2 & 2 & 2 & 2 & 1 & 1 & 1 & + & 2 & 2 & 10 & 100 \\
\hline & Primula vulgaris & E1 & + & 1 & 1 & + & 1 & . & . & + & . & . & 1 & + & 6 & 60 \\
\hline \multirow[t]{4}{*}{$\mathrm{AF}$} & Aremonio-Fagion & & & & & & & & & & & & & & & \\
\hline & Lamium orvala & E1 & 2 & 2 & 3 & 3 & 4 & 4 & 1 & 1 & 1 & 1 & 2 & 3 & 10 & 100 \\
\hline & Cardamine enneaphyllos & E1 & 2 & 2 & 2 & 2 & 2 & 1 & . & 2 & 2 & 2 & 2 & . & 9 & 90 \\
\hline & Cyclamen purpurascens & E1 & 1 & 1 & + & . & . & . & + & 1 & + & 1 & 1 & . & 7 & 70 \\
\hline \multirow[t]{9}{*}{ FS } & Fagetalia sylvaticae & & & & & & & & & & & & & & & \\
\hline & Carpinus betulus & $\mathrm{E} 3 \mathrm{~b}$ & 1 & 3 & 2 & 3 & 3 & 4 & 4 & 1 & 2 & 2 & 4 & 4 & 10 & 100 \\
\hline & Carpinus betulus & E3a & . & 1 & . & + & + & + & . & + & . & . & . & + & 5 & 50 \\
\hline & Carpinus betulus & $\mathrm{E} 2 \mathrm{~b}$ & + & . & . & . & $\cdot$ & . & 1 & . & . & . & . & . & 2 & 20 \\
\hline & Asarum europaeum subsp. caucasicum & E1 & 1 & + & 1 & + & 1 & 1 & 1 & 1 & 1 & 1 & 1 & + & 10 & 100 \\
\hline & Polygonatum multiflorum & E1 & 2 & + & 1 & 1 & 1 & 1 & 1 & 2 & 1 & 2 & + & 1 & 10 & 100 \\
\hline & Lathyrus vernus & E1 & 2 & 1 & + & 1 & $\cdot$ & + & 1 & + & + & + & + & . & 9 & 90 \\
\hline & Mercurialis perennis & E1 & 1 & + & + & 1 & 1 & 1 & + & + & + & . & + & 1 & 9 & 90 \\
\hline & Corydalis cava & E1 & 1 & + & 1 & + & 4 & 4 & 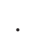 & + & + & . & . & 4 & 8 & 80 \\
\hline
\end{tabular}


Galeobdolon flavidum

Actaea spicata

Mycelis muralis

Galium laevigatum

Sambucus nigra

Salvia glutinosa

Campanula trachelium

Dryopteris filix-mas

Heracleum sphondylium

Sanicula europaea

Symphytum tuberosum

Cardamine bulbifera

Paris quadrifolia

Euphorbia dulcis

Melica nutans

Neottia nidus-avis

Galeobdolon montanum

Cardamine impatiens

Circaea lutetiana

Epipactis helleborine

Lonicera alpigena

Pulmonaria officinalis

$\mathrm{CO}$ Carpinion orientalis

Sesleria autumnalis

Carpinus orientalis?

QP

Quercetalia pubescenti-petraeae

Convallaria majalis

Lathyrus venetus

Ostrya carpinifolia

Ostrya carpinifolia

Fraxinus ornus

Fraxinus ornus

Fraxinus ornus

Fraxinus ornus

Helleborus multifidus subsp. istriacus*

Melittis melissophyllum

Tanacetum corymbosum

Asparagus tenuifolius

Mercurialis $x$ paxii

Cornus mas

Arabis turrita

Viola sepincola

Orchis pallens

Prunus mahaleb

Quercus cerris

Aristolochia lutea

Lathyrus niger

Hierochloë australis

Euonymus verrucosa

Quercus pubescens
E1

E1

E1

E

E2

E1

E1

E1

E1

E1

E1

E1

E1

E1

E1

E1

E1

E1

E1

E1

E2a

E1

E1

E3a

E1

E1

E3b

E3

E3a

E2b

E2a

E1

E1

E1

E1

E1

E1

E2b

E1

E1

E1

E2a

E3b

E1

E1

E1

E2a

E3a

\section{\begin{tabular}{llllllllll|ll|ll}
1 & 2 & 3 & 4 & 5 & 6 & 7 & 8 & 9 & 10 & 11 & 12 & Pr. & Fr.
\end{tabular}}

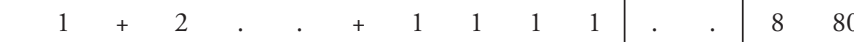

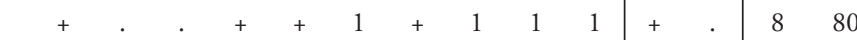

$++++.+1+.+1.970$

$1++1+.+5 .+2.70$

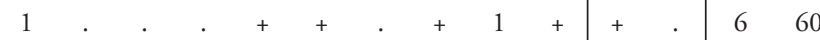

550

550

50

50

50

40

20

220

20

20

10

10

10

10

10

10

110

80

$2 \quad 20$

80

70

60

30

60

30

20

40

50

20

20

20

20

20

20

10

10

10

10

10

10

10

10 


\begin{tabular}{|c|c|c|c|c|c|c|c|c|c|c|c|c|c|c|c|c|}
\hline & Number of relevé (Zaporedna številka popisa) & & 1 & 2 & 3 & 4 & 5 & 6 & 7 & 8 & 9 & 10 & 11 & 12 & Pr. & Fr. \\
\hline & Sorbus aria (Aria edulis) & E3a & . & . & $\cdot$ & $\cdot$ & $\cdot$ & . & $\cdot$ & $\cdot$ & + & $\cdot$ & . & $\cdot$ & 1 & 10 \\
\hline \multirow[t]{23}{*}{ QF } & Querco-Fagetea & & & & & & & & & & & & & & & \\
\hline & Lonicera xylosteum & E2 & 1 & + & + & + & . & + & + & 1 & 1 & 1 & . & . & 9 & 9 \\
\hline & Hepatica nobilis & E1 & 1 & . & 1 & 1 & . & + & 1 & 1 & 1 & 1 & . & 1 & 8 & \\
\hline & Hedera helix & E3a & 1 & + & + & + & + & + & + & . & . & . & . & . & 7 & \\
\hline & Hedera helix & E1 & 1 & + & + & . & 1 & + & + & . & + & + & + & + & 8 & \\
\hline & Carex digitata & E1 & 1 & + & . & + & . & . & 1 & + & + & + & + & . & 7 & ( \\
\hline & Acer campestre & E1 & . & . & 1 & . & . & . & + & . & . & . & . & . & 2 & 20 \\
\hline & Anemone ranunculoides & E1 & + & . & . & + & + & 1 & + & 1 & . & . & + & 1 & 6 & 60 \\
\hline & Corylus avellana & E3a & . & . & . & $\cdot$ & . & . & . & . & . & + & . & . & 1 & 1 \\
\hline & Corylus avellana & $\mathrm{E} 2 \mathrm{~b}$ & 2 & . & . & . & . & 2 & . & + & 1 & 1 & . & + & 5 & 5 \\
\hline & Corylus avellana & E2a & + & . & . & $\cdot$ & . & . & . & . & $\cdot$ & . & . & . & 1 & 1 \\
\hline & Quercus petraea & $\mathrm{E} 3 \mathrm{~b}$ & $\mathrm{r}$ & 1 & 1 & . & + & . & . & . & . & . & + & . & 4 & 4 \\
\hline & Gagea lutea & E1 & . & $\cdot$ & . & + & + & + & . & + & . & . & 1 & . & 4 & 40 \\
\hline & Lathraea squamaria & E1 & . & + & $\mathrm{r}$ & . & + & . & . & . & . & . & . & + & 3 & 30 \\
\hline & Viola riviniana & E1 & + & $\cdot$ & . & + & . & . & . & . & . & . & + & . & 2 & 2 \\
\hline & Stellaria holostea & E1 & . & . & . & . & . & . & . & + & + & + & 2 & . & 3 & 30 \\
\hline & Ranunculus ficaria & E1 & . & . & . & . & 1 & 1 & . & . & . & . & . & 1 & 2 & 2 \\
\hline & Anemone nemorosa & E1 & . & . & . & $\cdot$ & . & . & . & + & . & + & 1 & . & 2 & 2 \\
\hline & Moehringia trinervia & E1 & . & . & . & $\cdot$ & + & . & . & . & $\cdot$ & . & + & . & 1 & 1 \\
\hline & Dactylis glomerata subsp. lobata (D. polygama) & E1 & . & . & . & $\cdot$ & . & . & . & + & $\cdot$ & . & + & . & 1 & 10 \\
\hline & Ulmus minor & $\mathrm{E} 2 \mathrm{a}$ & . & $\cdot$ & . & $\cdot$ & . & . & $\cdot$ & + & $\cdot$ & . & . & . & 1 & 10 \\
\hline & Carex pilosa & E1 & . & . & . & $\cdot$ & . & . & $\cdot$ & . & $\cdot$ & . & + & . & . & \\
\hline & Listera ovata & E1 & . & . & . & . & . & . & $\cdot$ & . & . & . & . & + & . & \\
\hline \multirow[t]{4}{*}{ VP } & Vaccinio-Piceetea & & & & & & & & & & & & & & & \\
\hline & Maianthemum bifolium & E1 & . & . & . & $\cdot$ & . & . & . & + & . & + & 2 & . & 2 & 2 \\
\hline & Solidago virgaurea & E1 & . & · & . & $\cdot$ & . & . & . & + & $\cdot$ & + & . & . & 2 & 2 \\
\hline & Hieracium murorum & E1 & . & $\cdot$ & . & $\cdot$ & . & . & . & + & · & . & . & . & 1 & 1 \\
\hline \multirow[t]{3}{*}{$\mathrm{RP}$} & Rhmano-Prunetea & & & & & & & & & & & & & & & \\
\hline & Euonymus europaea & E2 & . & . & . & $\cdot$ & . & . & $\cdot$ & + & + & . & . & . & 2 & 2 \\
\hline & Berberis vulgaris & E2a & . & . & . & . & . & . & . & . & + & . & . & . & 1 & 1 \\
\hline \multicolumn{17}{|c|}{ MuA Mulgedio-Aconitetea, Betulo-Alnetea } \\
\hline & Senecio nemorensis & E1 & . & . & . & . & . & . & . & + & + & . & . & . & 2 & 20 \\
\hline & Ribes alpinum & E2a & . & · & . & $\cdot$ & . & . & . & 1 & $\cdot$ & . & . & . & 1 & 1 \\
\hline & Senecio ovatus & E1 & . & $\cdot$ & . & . & . & . & . & . & + & $\cdot$ & . & $\cdot$ & 1 & 1 \\
\hline \multirow[t]{5}{*}{ EA } & Epilobietea angustifolii & & & & & & & & & & & & & & & \\
\hline & Solanum dulcamara & E1 & . & . & . & $\cdot$ & . & . & $\cdot$ & . & . & + & . & . & 1 & 1 \\
\hline & Galio-Urticetea & & & & & & & & & & & & & & & \\
\hline & Urtica dioica & E1 & . & . & . & $\cdot$ & . & + & $\cdot$ & . & + & . & . & . & 2 & 2 \\
\hline & Geum urbanum & E1 & . & . & . & $\cdot$ & . & . & . & . & . & . & + & $\cdot$ & . & \\
\hline \multirow[t]{2}{*}{ SM } & Stellarietea mediae & & & & & & & & & & & & & & & \\
\hline & Stellaria neglecta & E1 & . & . & . & $\cdot$ & + & + & $\cdot$ & . & . & . & . & . & 2 & 20 \\
\hline \multirow[t]{4}{*}{ TG } & Trifolio-Geranietea & & & & & & & & & & & & & & & \\
\hline & Lilium carniolicum & E1 & . & + & . & $\cdot$ & . & . & $\cdot$ & $\mathrm{r}$ & . & . & . & . & 2 & 20 \\
\hline & Viola odorata & E1 & . & . & . & . & + & . & $\cdot$ & . & . & . & . & + & 1 & 10 \\
\hline & Melampyrum velebiticum & E1 & . & · & . & $\cdot$ & . & . & $\cdot$ & . & . & + & . & . & 1 & 1 \\
\hline \multirow[t]{2}{*}{$\mathrm{FB}$} & Festuco-Brometea & & & & & & & & & & & & & & & \\
\hline & Allium carinatum subsp. carinatum & E1 & . & . & . & · & . & $\cdot$ & + & . & . & . & . & . & 1 & 10 \\
\hline \multirow[t]{2}{*}{ KC } & Koelerio-Corynephoretea & & & & & & & & & & & & & & & \\
\hline & Cardaminopsis arenosa & E1 & $\cdot$ & $\cdot$ & . & $\cdot$ & . & $\cdot$ & $\cdot$ & + & . & . & . & . & 1 & \\
\hline
\end{tabular}




\begin{tabular}{|c|c|c|c|c|c|c|c|c|c|c|c|c|c|c|c|c|}
\hline & Number of relevé (Zaporedna številka popisa) & & 1 & 2 & 3 & 4 & 5 & 6 & 7 & 8 & 9 & 10 & 11 & 12 & Pr. & Fr. \\
\hline \multirow[t]{4}{*}{ MA } & \multicolumn{16}{|l|}{ Molinio-Arrbenatheretea } \\
\hline & Veronica chamaedrys & E1 & . & . & + & . & . & . & . & . & . & . & . & . & 1 & 10 \\
\hline & Anthriscus sylvestris & E1 & . & . & . & . & . & + & . & . & . & . & . & . & 1 & 10 \\
\hline & Muscari botryoides & E1 & . & . & . & . & . & . & $\mathrm{r}$ & . & . & . & . & . & 1 & 10 \\
\hline \multirow[t]{3}{*}{ Cy } & \multicolumn{16}{|l|}{ Cystopteridion fragilis } \\
\hline & Moehringia muscosa & E1 & 1 & + & . & . & . & . & . & + & . & . & . & . & 3 & 30 \\
\hline & Cystopteris fragilis & E1 & + & . & . & . & . & . & . & . & . & . & . & . & 1 & 10 \\
\hline \multirow[t]{2}{*}{ PS } & \multicolumn{16}{|c|}{ Physoplexido-Saxifragion, Potentilletalia caulescentis } \\
\hline & Campanula pyramidalis & E1 & . & . & + & . & . & . & . & . & . & . & . & . & 1 & 10 \\
\hline \multirow[t]{6}{*}{ AT } & \multicolumn{16}{|l|}{ Asplenietea trichomanis } \\
\hline & Asplenium trichomanes & E1 & . & . & . & . & . & . & . & + & + & + & . & . & 3 & 30 \\
\hline & Polypodium vulgare & E1 & . & . & . & . & . & . & . & + & + & + & . & . & 3 & 30 \\
\hline & Asplenium adiantum-nigrum & E1 & . & + & . & . & . & . & . & . & . & . & . & . & 1 & 10 \\
\hline & Polypodium interjectum & E1 & . & . & . & . & . & . & . & + & . & . & . & . & 1 & 10 \\
\hline & Asplenium ruta-muraria & E1 & . & . & . & . & . & . & . & + & . & . & . & . & 1 & 10 \\
\hline \multirow[t]{14}{*}{ ML } & \multicolumn{16}{|l|}{ Mosses and lichens (Mahovi in lišaji) } \\
\hline & Isothecium alopecuroides & E0 & 1 & . & 1 & + & 1 & . & 1 & 2 & 1 & 2 & 1 & . & 8 & 80 \\
\hline & Brachythecium rutabulum & E0 & 1 & . & 1 & + & 1 & 1 & . & 1 & 1 & 1 & + & . & 8 & 80 \\
\hline & Ctenidium molluscum & E0 & 1 & . & 1 & 1 & . & . & 1 & 2 & 2 & 2 & + & . & 7 & 70 \\
\hline & Neckera crispa & E0 & 1 & . & + & + & 1 & . & . & + & + & 2 & . & . & 7 & 70 \\
\hline & Thamnobryum alopecurum & E0 & + & $\cdot$ & + & 1 & 1 & . & . & 2 & 1 & 1 & 1 & . & 7 & 70 \\
\hline & Plagiomnium undulatum & E0 & 2 & + & 1 & + & + & + & . & + & . & . & . & . & 7 & 70 \\
\hline & Homalothecium lutescens & E0 & + & . & 1 & . & . & + & 1 & + & 1 & . & 1 & + & 6 & 60 \\
\hline & Peltigera canina & E0 & + & . & + & . & . & + & . & + & + & . & . & . & 5 & 50 \\
\hline & Anomodon viticulosus & E0 & . & + & . & . & . & . & . & . & 1 & 2 & + & + & 3 & 30 \\
\hline & Neckera complanata & E0 & . & . & + & 1 & . & . & 1 & . & . & . & . & . & 3 & 30 \\
\hline & Anomodon attenuatus & E0 & . & . & . & . & . & . & . & 1 & 1 & + & + & . & 3 & 30 \\
\hline & Eurhynchium striatum & E0 & 1 & . & . & . & . & . & . & . & . & . & . & . & 1 & 10 \\
\hline & Polytrichum formosum & E0 & . & . & . & . & . & . & . & + & . & . & . & . & 1 & 10 \\
\hline
\end{tabular}

\section{Legend - Legenda}

Relevés 1-10: Corydalido-Aceretum pseudoplatani

Relevés 11-12: Asaro-Carpinetum betuli

A Limestone - apnenec

Gr Gravel - grušč

Re Rendzina - rendzina

Co Colluvial soil - koluvialna tla

$\mathrm{Rj}$ Calcareous brown soil - rjava pokarbonatna tla

Pr. Presence (number of relevés in which the species is presented) - število popisov, v katerih se pojavlja vrsta

Fr. Frequency in $\%$ - frekvenca $v \%$

? determination is unreliable - določitev ni zanesljiva

Helleborus multifidus subsp. istriacus* (incl. Helleborus dumetorum subsp. istriacus) - Rottensteiner (2016) 
Table 2 (Tabela 2): Fraxino orni-Aceretum pseudoplatani ass. nov.

\section{Number of relevé (Zaporedna števillka popisa)}

Database number of relevé

(Delovna številka popisa)

Elevation in $\mathrm{m}$ (Nadmorska višina $\mathrm{v} \mathrm{m}$ )

Aspect (Lega)

Slope in degrees (Nagib v stopinjah)

Parent material (Matična podlaga)

Soil (Tla)

Stoniness in \% (Kamnitost v \%)

Cover in \% (Zastiranje v \%):

$\begin{array}{lllllllllllllll}\text { Upper tree layer (Zgornja drevesna plast) } & \text { E3b } & 80 & 70 & 70 & 60 & 70 & 60 & 60 & 70 & 70 & 70 & 80 & 70 & 70\end{array}$

Lower tree layer (Spodnja drevesna plasti)

Shrub layer (Grmovna plast)

Herb layer (Zeliščna plast)

Moss layer (Mahovna plast)

Max. tree diameter (Maks. premer dreves)

Max. tree height (Maks. višina dreves)

Number of species (Število vrst)

Relevé area (Velikost popisne ploskve)

Date of taking relevé (Datum popisa)

Locality (Nahajališče)

Quadrant (Kvadrant)

Coordinate (Koordinate) GK Y (D-48)

Coordinate (Koordinate) GK X (D-48)

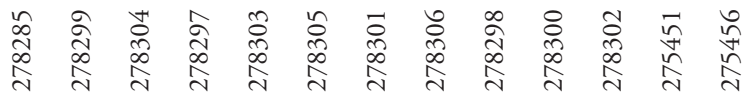

$\begin{array}{lllllllllllll}235 & 175 & 180 & 150 & 170 & 200 & 175 & 185 & 140 & 170 & 180 & 275 & 330\end{array}$ NNE N NNE NE SSW $N$ N $S$ S $\quad S \quad S E$ SE NE NE $\begin{array}{lllllllllllll}30 & 25 & 5 & 0-10 & 15 & 40 & 5 & 20 & 0-35 & 35 & 25 & 45 & 1\end{array}$ $\begin{array}{lllllllllllll}\text { A,Fl } & \text { Ps } & \text { Ta } & \text { Ps } & \text { Ta } & \text { Gr } & \text { Ps } & \text { Ps } & \text { Br } & \text { Ps } & \text { Ps } & \text { L } & \text { Fl }\end{array}$ Eu Co Co Co Co Co Co Co Re Re Re Co Eu $\begin{array}{lllllllllllll}30 & 70 & 30 & 30 & 60 & 40 & 20 & 70 & 40 & 80 & 80 & 0 & 0\end{array}$

\section{Diagnostic species of the association (Diagnostične vrste asociacije)}

TA Ulmus glabra

TA Ulmus glabra

$\mathrm{E} 3 \mathrm{a} 1++.+2 .+2 .+2 .+54$

TA Ulmus glabra

$\mathrm{E} 2 \mathrm{~b} 1+1++1++++1+.1292$

TA Ulmusglabra

$\mathrm{E} 2 \mathrm{a} 1+++.+++.+{ }_{+}+862$

AF Lamium orvala

E1

CO Ruscus aculeatus

$\begin{array}{llllllllllllllll}\mathrm{E} 1 & 3 & 3 & 4 & 4 & 3 & 3 & 3 & 2 & 3 & 3 & 3 & 3 & 4 & 13 & 100\end{array}$

QP Fraxinus ornus

$\begin{array}{llllllllllllll}\mathrm{E} 2 \mathrm{a} & 4 & 4 & 4 & 3 & 3 & 3 & 3 & 2 & 3 & + & 12 & & \end{array}$ $\mathrm{E} 3 \mathrm{~b}+.+5 .+2 .+1+431$ 


\begin{tabular}{|c|c|c|c|c|c|c|c|c|c|c|c|c|c|c|c|c|c|}
\hline & Number of relevé (Zaporedna številka popisa) & & 1 & 2 & 3 & 4 & 5 & 6 & 7 & 8 & 9 & 10 & 11 & 12 & 13 & Pr. & Fr. \\
\hline $\mathrm{QP}$ & Fraxinus ornus & E3a & . & . & . & . & . & . & . & . & . & . & + & . & . & 1 & 8 \\
\hline QP & Fraxinus ornus & $\mathrm{E} 2 \mathrm{~b}$ & + & . & . & . & . & . & . & . & . & . & 1 & . & . & 2 & 15 \\
\hline QP & Fraxinus ornus & $\mathrm{E} 2 \mathrm{a}$ & . & + & + & + & . & $\cdot$ & + & 1 & . & + & 1 & . & . & 7 & 54 \\
\hline $\mathrm{CO}$ & Asparagus acutifolius & $\mathrm{E} 2 \mathrm{a}$ & . & + & . & . & + & $\cdot$ & . & . & + & . & + & . & . & 4 & 31 \\
\hline \multirow[t]{16}{*}{ TA } & Tilio-Acerion & & & & & & & & & & & & & & & & \\
\hline & Polystichum setiferum & E1 & + & 3 & 1 & + & 1 & 4 & + & 1 & 1 & + & . & + & . & 11 & 85 \\
\hline & Phyllitis scolopendrium & E1 & 2 & + & + & 1 & 1 & 3 & + & 2 & 1 & 1 & 1 & . & . & 11 & 85 \\
\hline & Lunaria rediviva & E1 & . & 1 & 2 & 4 & 4 & 4 & 4 & 3 & 2 & + & . & . & . & 9 & 69 \\
\hline & Isopyrum thalictroides & E1 & 3 & 2 & + & 1 & . & $\cdot$ & 1 & + & + & 1 & . & . & . & 8 & 62 \\
\hline & Geranium robertianum & E1 & + & . & . & . & . & + & . & . & + & 2 & 2 & . & . & 5 & 38 \\
\hline & Tilia platyphyllos & E3b & . & + & . & . & . & $\cdot$ & . & . & 1 & . & . & . & . & 2 & 15 \\
\hline & Tilia platyphyllos & E3a & . & . & + & . & . & $\cdot$ & . & . & . & . & + & . & . & 2 & 15 \\
\hline & Tilia platyphyllos & $\mathrm{E} 2 \mathrm{~b}$ & . & . & . & . & . & $\cdot$ & . & . & . & . & . & + & . & 1 & 8 \\
\hline & Aruncus dioicus & E1 & . & + & . & . & . & $\cdot$ & . & . & + & . & . & . & . & 2 & 15 \\
\hline & Juglans regia & $\mathrm{E} 3 \mathrm{~b}$ & . & . & . & . & . & . & . & . & $\mathrm{r}$ & . & . & . & $\mathrm{r}$ & 2 & 15 \\
\hline & Juglans regia & $\mathrm{E} 2 \mathrm{a}$ & + & . & . & + & . & . & . & . & . & . & . & . & . & 2 & 15 \\
\hline & Polystichum aculeatum & E1 & + & . & . & . & . & $\cdot$ & . & . & . & . & . & . & . & 1 & 8 \\
\hline & Tilia cordata & $\mathrm{E} 3 \mathrm{~b}$ & . & . & + & . & . & . & . & . & . & . & . & . & . & 1 & 8 \\
\hline & Dryopteris affinis & E1 & . & . & . & . & + & . & . & . & . & . & . & . & . & 1 & 8 \\
\hline & Tephroseris pseudocrispa & E1 & . & . & . & . & . & . & . & . & . & . & . & 1 & . & 1 & 8 \\
\hline \multirow[t]{5}{*}{$\mathrm{EC}$} & Erythronio-Carpinion & & & & & & & & & & & & & & & & \\
\hline & Galanthus nivalis & E1 & 2 & . & . & 2 & 2 & 3 & 2 & 2 & 2 & 2 & 1 & 1 & + & 11 & 85 \\
\hline & Helleborus odorus & E1 & 1 & + & 1 & + & . & $\cdot$ & + & + & + & + & . & . & 1 & 9 & 69 \\
\hline & Crocus vernus subsp. vernus* & E1 & . & + & + & . & . & . & . & + & . & + & . & . & 2 & 5 & 38 \\
\hline & Primula vulgaris & E1 & . & . & . & . & . & $\cdot$ & . & . & . & . & . & . & + & 1 & 8 \\
\hline \multirow[t]{4}{*}{$\mathrm{AF}$} & Aremonio-Fagion & & & & & & & & & & & & & & & & \\
\hline & Cardamine enneaphyllos & E1 & 2 & . & 2 & 1 & 2 & + & 2 & + & 1 & + & 1 & . & . & 10 & 77 \\
\hline & Hacquetia epipactis & E1 & 1 & 1 & + & . & . & $\cdot$ & 1 & . & + & . & . & + & 1 & 7 & 54 \\
\hline & Cyclamen purpurascens & E1 & + & . & . & + & . & $\cdot$ & . & + & + & . & + & . & . & 5 & 38 \\
\hline \multirow[t]{3}{*}{ AI } & Alnion incanae & & & & & & & & & & & & & & & & \\
\hline & Alnus glutinosa & E3b & . & . & . & . & . & . & . & . & . & . & . & . & + & 1 & 8 \\
\hline & Festuca gigantea & E1 & . & . & . & . & . & . & . & . & . & . & . & . & + & 1 & 8 \\
\hline \multirow[t]{15}{*}{ FS } & Fagetalia sylvaticae & & & & & & & & & & & & & & & & \\
\hline & Mercurialis perennis & E1 & 2 & 2 & 1 & 1 & 1 & 2 & 1 & 2 & 1 & 1 & 1 & + & + & 13 & 100 \\
\hline & Asarum europaeum subsp. caucasicum & E1 & + & 1 & + & 1 & + & $\cdot$ & + & + & + & + & + & 1 & + & 12 & 92 \\
\hline & Corydalis cava & E1 & 1 & + & + & 3 & 3 & 2 & 2 & 2 & 2 & 1 & + & . & . & 11 & 85 \\
\hline & Sambucus nigra & $\mathrm{E} 2 \mathrm{~b}$ & + & + & + & + & + & + & . & + & 1 & 1 & 1 & + & . & 11 & 85 \\
\hline & Sambucus nigra & $\mathrm{E} 2 \mathrm{a}$ & . & . & . & + & + & . & . & + & + & + & . & . & . & 5 & 38 \\
\hline & Cardamine bulbifera & E1 & 1 & + & 1 & + & 1 & 1 & + & + & 1 & 1 & 1 & . & . & 11 & 85 \\
\hline & Polygonatum multiflorum & E1 & 1 & 1 & . & + & . & . & + & . & + & + & + & 1 & 1 & 9 & 69 \\
\hline & Salvia glutinosa & E1 & . & + & + & . & + & . & . & + & + & + & + & + & 2 & 9 & 69 \\
\hline & Symphytum tuberosum & E1 & . & + & 1 & . & . & . & + & . & $\mathrm{r}$ & + & + & 1 & 1 & 8 & 62 \\
\hline & Mycelis muralis & E1 & 1 & + & . & . & + & 1 & . & . & . & 1 & 1 & . & + & 7 & 54 \\
\hline & Lilium martagon & E1 & + & + & + & + & . & + & + & . & . & $r$ & . & . & . & 7 & 54 \\
\hline & Pulmonaria officinalis & E1 & + & + & + & . & . & 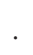 & . & + & + & . & + & + & . & 7 & 54 \\
\hline & Carpinus betulus & $\mathrm{E} 3 \mathrm{~b}$ & . & 1 & + & + & . & e & . & 2 & + & . & + & . & . & 6 & 46 \\
\hline & Carpinus betulus & E3a & + & . & . & . & . & 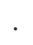 & . & . & . & + & . & . & . & 2 & 15 \\
\hline
\end{tabular}


Carpinus betulus

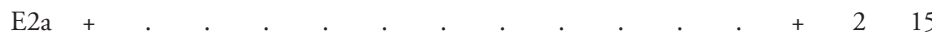

Carpinus betulus

E1

Galeobdolon flavidum

Viola reichenbachiana

Cardamine impatiens

Lathyrus vernus

Dryopteris filix-mas

Actaea spicata

Galeobdolon montanum

Brachypodium sylvaticum

Euphorbia dulcis

Circaea lutetiana

Fagus sylvatica

Fagus sylvatica

Fagus sylvatica

Fagus sylvatica

Prunus avium

Carex sylvatica

Scrophularia nodosa

Prenanthes purpurea

$\mathrm{E} 11+\ldots+.+1 .+1.646$

$\mathrm{E} 1+++.+.+.+2 .+646$

$\mathrm{E} 1+.+.+.+1 .+5.58$

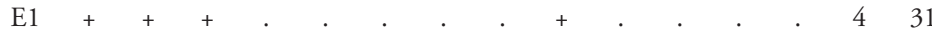

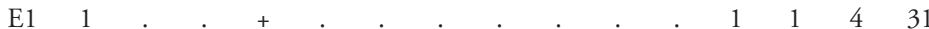

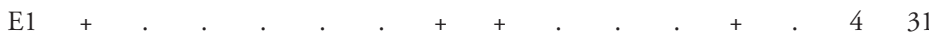

$\mathrm{E} 1 .+2++.+.+4.41$

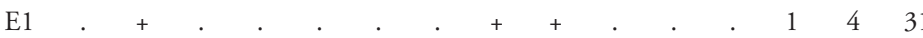

$\mathrm{E} 1+\mathrm{r} \cdot+\cdot \mathrm{r}^{2} \cdot 3$

$\mathrm{E} 1++\ldots+23$

E3a $+r-r$

$\mathrm{E} 2 \mathrm{~b} . \mathrm{r} \cdot \ldots+215$

18

E1

E3b

E1

E1

E1

CO Carpinion orientalis

Sesleria autumnalis

E1 $\quad \mathrm{r}$

8

QP Quercetalia pubescenti-petraeae

Lathyrus venetus

Ostrya carpinifolia

Cornus mas

Arabis turrita

Helleborus multifidus subsp. istriacus

Tamus communis

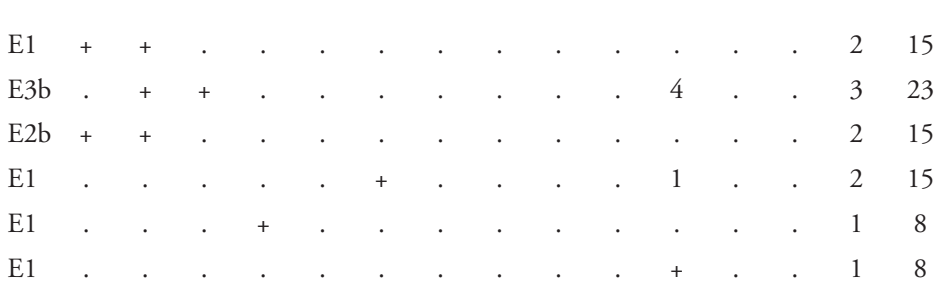

QR Quercetalia roboris

Castanea sativa

E3b

Castanea sativa

E1

Rubus hirtus

QF Querco-Fagetea

Hedera helix

Hedera helix

Aegopodium podagraria

Anemone nemorosa

Corylus avellana

Corylus avellana

Corylus avellana

Hepatica nobilis

Ranunculus ficaria

Clematis vitalba

Clematis vitalba

Acer campestre

Acer campestre

\begin{tabular}{|c|c|c|c|c|c|c|c|c|c|c|c|c|c|c|c|}
\hline E3a & 1 & 1 & 1 & 1 & & 1 & + & 1 & 1 & 1 & + & 1 & + & 12 & \\
\hline E1 & + & . & + & + & & 1 & . & + & 1 & + & 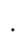 & 1 & 1 & 9 & 6 \\
\hline E1 & + & + & 1 & 2 & 1 & 1 & + & + & 1 & 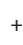 & r. & + & 1 & 12 & \\
\hline E1 & 1 & + & 1 & + & + & $\cdot$ & 1 & . & + & + & . & + & 1 & 10 & \\
\hline E3a & 1 & . & . & . & $\cdot$ & . & . & $\cdot$ & . & + & . & . & . & 2 & \\
\hline E2 & 1 & 1 & 1 & 1 & 1 & . & + & . & 1 & 1 & . & 1 & . & 9 & \\
\hline E1 & . & . & . & . & ${ }^{\circ}$ & $\cdot$ & . & . & $\cdot$ & + & & . & + & 2 & \\
\hline E1 & 1 & + & + & + & . & . & . & . & + & . & 1 & . & . & 6 & \\
\hline E1 & + & . & . & + & . & . & . & - & 1 & + & $\cdot$ & . & + & 6 & \\
\hline E3a & . & . & . & . & $\cdot$ & . & . & . & . & + & . & . & . & 1 & \\
\hline E2 & . & . & . & . & + & . & + & + & . & + & . & + & + & 6 & \\
\hline E3b & + & . & . & 1 & . & . & + & + & 1 & + & . & . & . & 6 & \\
\hline E3a & + & + & & . & . & & . & . & + & + & + & . & . & 5 & \\
\hline
\end{tabular}


Acer campestre

Acer campestre

Crataegus laevigata

Carex digitata

E2

Cerastium sylvaticum

E1

Lathraea squamaria

E1

Quercus petraea

E1

Quercus petraea

E3b

E1

Malus sylvestris

Veratrum nigrum

Gagea lutea

Scilla bifolia

Moehringia trinervia

VP Vaccinio-Piceetea

Oxalis acetosella

Aposeris foetida

Picea abies

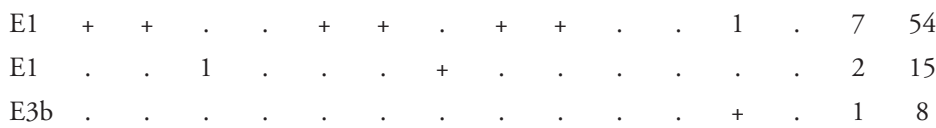

RP Rhmano-Prunetea

Robinia pseudoacacia

$\mathrm{E} 3 \mathrm{~b}+\ldots .2$

Robinia pseudoacacia

E3a

Robinia pseudoacacia

E2a

Euonymus europaea

E2

Crataegus monogyna

MuA Mulgedio-Aconitetea, Betulo-Alnetea

Aconitum lycoctonum

Athyrium filix-femina

Senecio nemorensis

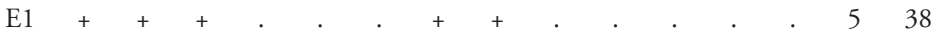

$\mathrm{E} 1+.+.+.+2 .+431$

E1

EA Epilobietea angustifolii

Carpesium cernuum

Galeopsis pubescens

Physalis alkekengi

Arctium nemorosum

Stachys sylvatica

GU Galio-Urticetea

Geum urbanum

Parietaria officinalis

Urtica dioica

E1

E1

E1

E1

E1

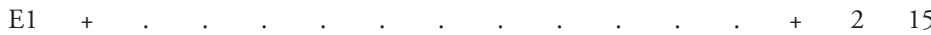

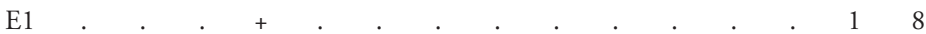

E1 . . . . . . . . . 18

SM Stellarietea mediae

Galium aparine

E1

Erigeron annuus

Cy Cystopteridion fragilis

Moehringia muscosa

PS Physoplexido-Saxifragion, Potentilletalia caulescentis Campanula pyramidalis

E1

AT Asplenietea trichomanis

Asplenium trichomanes

Polypodium interjectum

Polypodium vulgare

$\mathrm{E} 1++.++.+2 .+1.646$

$\mathrm{E} 1++.+2 .+5 .+538$ 


\title{
$\begin{array}{llllllllllllllllll}\text { Number of relevé (Zaporedna številka popisa) } & 1 & 2 & 3 & 4 & 5 & 6 & 7 & 8 & 9 & 10 & 11 & 12 & 13 & \text { Pr. } & \text { Fr. }\end{array}$
}

ML Mosses and lichens (Mahovi in lišaji)

\begin{tabular}{|c|c|c|c|c|c|c|c|c|c|c|c|c|c|c|c|}
\hline Thamnobryum alopecurum & E0 & 3 & 3 & 1 & 3 & 3 & 3 & 2 & . & 2 & 3 & 3 & + & 11 & 85 \\
\hline Isothecium alopecuroides & E0 & 1 & 2 & 1 & + & 2 & . & 1 & 2 & 1 & . & 2 & . & 9 & 69 \\
\hline Ctenidium molluscum & E0 & . & + & . & + & . & . & . & . & 1 & 2 & 3 & . & 5 & 38 \\
\hline Anomodon viticulosus & E0 & + & . & . & . & . & . & . & 1 & 1 & 1 & 1 & . & 5 & 38 \\
\hline Neckera complanata & E0 & . & . & 1 & . & . & . & . & 2 & . & . & 1 & . & 3 & 23 \\
\hline Brachythecium rutabulum & E0 & . & . & . & 2 & 1 & . & . & . & 1 & . & . & . & 3 & 23 \\
\hline Anomodon attenuatus & E0 & . & . & . & . & . & . & 1 & + & . & . & . & . & 2 & 15 \\
\hline Neckera crispa & E0 & . & . & . & . & . & . & . & . & + & 1 & . & . & 2 & 15 \\
\hline Homalothecium lutescens & E0 & . & . & . & . & . & . & . & . & . & 1 & 1 & . & 2 & 15 \\
\hline Fissidens taxifolius & E0 & + & . & . & . & . & . & . & . & . & . & . & . & 1 & 8 \\
\hline Plagiomnium undulatum & E0 & + & . & . & . & . & . & . & . & . & . & . & . & 1 & 8 \\
\hline Conocephalum conicum & E0 & . & + & . & . & . & . & . & . & . & . & . & . & 1 & 8 \\
\hline Thuidium delicatulum & E0 & . & . & . & 1 & . & . & . & . & . & . & . & . & 1 & 8 \\
\hline
\end{tabular}

\section{Legend - Legenda}

\author{
A Limestone - apnenec \\ Fl Flysch - fliš \\ Gr Gravel - grušč \\ Ro Rockslide - podorno skalovje \\ Ta Talus - vršaj \\ Br Breccia - breča \\ L Marlstone - laporovec \\ Re Rendzina - rendzina \\ Co Colluvial soil - koluvialna tla \\ Eu Eutric brown soli - evtrična rjava tla \\ Pr. Presence (number of relevés in which the species is presented) - število popisov, v katerih se pojavlja vrsta \\ Fr. Frequency in $\%$ - frekvenca $v \%$
}

Crocus vernus subsp. vernus* is according to new findings Crocus heuffellianus Herb. (Peruzzi 2016) 
Tabla 3 (Tabela 3): Paeonio officinalis-Tilietum platyphylli saxifragetosum rotundifoliae, castaneetosum.

\begin{tabular}{|c|c|c|c|c|c|c|c|c|c|c|c|c|c|c|}
\hline \multicolumn{2}{|l|}{ Number of relevé (Zaporedna številka popisa) } & 1 & 2 & 3 & 4 & 5 & 6 & 7 & 8 & 9 & 10 & 11 & 12 & 13 \\
\hline \multicolumn{2}{|l|}{$\begin{array}{l}\text { Database number of relevé } \\
\text { (Delovna številka popisa) }\end{array}$} & 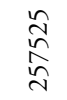 & 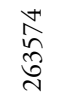 & 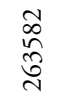 & 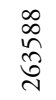 & 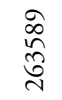 & $\begin{array}{l}\hat{\infty} \\
\hat{\imath} \\
\text { ते }\end{array}$ & 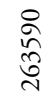 & $\begin{array}{c}\hat{\Sigma} \\
\hat{\tilde{\sigma}} \\
\text { }\end{array}$ & 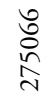 & $\begin{array}{l}\stackrel{R}{S} \\
\stackrel{n}{n} \\
\stackrel{n}{N}\end{array}$ & 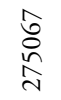 & $\begin{array}{l}\infty \\
\stackrel{\infty}{0} \\
\stackrel{n}{N}\end{array}$ & 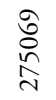 \\
\hline \multicolumn{2}{|l|}{ Elevation in $\mathrm{m}$ (Nadmorska višina $\mathrm{v} \mathrm{m})$} & 740 & 750 & 735 & 755 & 755 & 740 & 740 & 750 & 335 & 335 & 340 & 310 & 320 \\
\hline \multicolumn{2}{|l|}{ Aspect (Lega) } & SWW & NW & NWW & NW & NW & NWW & NW & W & $\mathrm{NE}$ & $\mathrm{NE}$ & $\mathrm{NE}$ & $\mathrm{NE}$ & $\mathrm{NE}$ \\
\hline \multicolumn{2}{|l|}{ Slope in degrees (Nagib v stopinjah) } & 30 & 30 & 40 & 35 & 35 & 30 & 25 & 30 & 20 & 35 & 35 & 35 & 35 \\
\hline \multicolumn{2}{|l|}{ Parent material (Matična podlaga) } & Gr & Gr & A & $\mathrm{Gr}$ & Gr & $\mathrm{Gr}$ & $\mathrm{Gr}$ & $\mathrm{AL}$ & $\mathrm{AL}$ & $\mathrm{AL}$ & $\mathrm{AL}$ & $\mathrm{AL}$ & $\mathrm{AL}$ \\
\hline \multicolumn{2}{|l|}{ Soil (Tla) } & Co & Co & $\operatorname{Re}$ & Co & Co & Co & Co & $\operatorname{Re}$ & $\operatorname{Re}$ & $\operatorname{Re}$ & $\operatorname{Re}$ & Co & $\operatorname{Re}$ \\
\hline \multicolumn{2}{|l|}{ Stoniness in \% (Kamnitost v \%) } & 40 & 60 & 40 & 30 & 30 & 40 & 30 & 20 & 70 & 70 & 70 & 60 & 30 \\
\hline \multicolumn{15}{|l|}{ Cover in \% (Zastiranje v \%): } \\
\hline Upper tree layer (Zgornja drevesna plast) & $\mathrm{E} 3 \mathrm{~b}$ & 80 & 70 & 80 & 70 & 70 & 80 & 80 & 80 & 80 & 80 & 80 & 80 & 70 \\
\hline Lower tree layer (Spodnja drevesna plasti) & E3a & 10 & 10 & 10 & 20 & 10 & 10 & 10 & 10 & 10 & 5 & 10 & 10 & 10 \\
\hline Shrub layer (Grmovna plast) & E2 & 10 & 10 & 10 & 10 & 10 & 5 & 10 & 10 & 10 & 20 & 20 & 20 & 20 \\
\hline Herb layer (Zeliščna plast) & E1 & 50 & 70 & 60 & 70 & 70 & 60 & 70 & 80 & 30 & 30 & 60 & 60 & 70 \\
\hline Moss layer (Mahovna plast) & E0 & 10 & 50 & 30 & 30 & 10 & 20 & 30 & 10 & 20 & 20 & 20 & 5 & 5 \\
\hline Max. tree diameter (Maks. premer dreves) & $\mathrm{cm}$ & 35 & 35 & 30 & 30 & 35 & 35 & 50 & 30 & 50 & 40 & 60 & 40 & 60 \\
\hline Max. tree height (Maks. višina dreves) & $\mathrm{m}$ & 19 & 18 & 16 & 15 & 20 & 20 & 25 & 17 & 25 & 25 & 25 & 20 & 24 \\
\hline Number of species (Število vrst) & & 66 & 66 & 80 & 65 & 61 & 77 & 66 & 52 & 40 & 48 & 44 & 47 & 55 \\
\hline Relevé area (Velikost popisne ploskve) & $\mathrm{m}^{2}$ & 400 & 400 & 400 & 400 & 400 & 400 & 400 & 400 & 400 & 400 & 400 & 400 & 400 \\
\hline Date of taking relevé (Datum popisa) & & 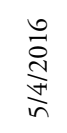 & 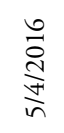 & 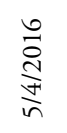 & 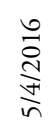 & 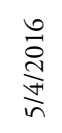 & 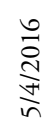 & 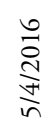 & 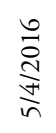 & 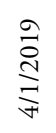 & 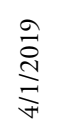 & 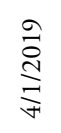 & $\underset{\vec{\forall}}{\stackrel{\vec{ٍ}}{\rightrightarrows}}$ & 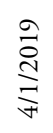 \\
\hline Locality (Nahajališče) & & 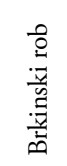 & 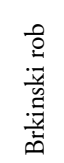 & 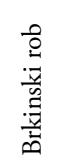 & 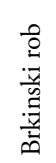 & 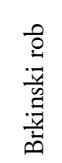 & 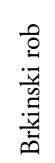 & 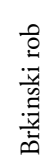 & 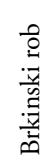 & 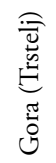 & 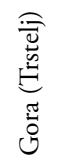 & 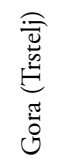 & 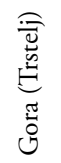 & 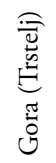 \\
\hline Quadrant (Kvadrant) & & $\stackrel{m}{\approx}$ & $\stackrel{m}{\approx}$ & $\stackrel{m}{\approx}$ & $\stackrel{m}{\approx}$ & $\stackrel{m}{\tilde{n}}$ & $\stackrel{n}{\approx}$ & $\stackrel{n}{\approx}$ & $\stackrel{m}{\approx}$ & $\underset{\substack{\sigma \\
+\\
\sigma}}{+}$ & $\underset{\stackrel{\nabla}{\infty}}{\stackrel{+}{\sigma}}$ & 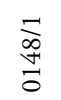 & $\begin{array}{l}\vec{\infty} \\
\stackrel{\infty}{+1} \\
0\end{array}$ & $\begin{array}{l}0 \\
0 \\
0 \\
\\
\end{array}$ \\
\hline Coordinate (Koordinate) GK Y (D-48) & $\mathrm{m}$ & 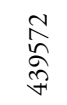 & 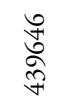 & 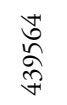 & 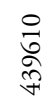 & $\begin{array}{l}\text { } \\
\text { ळे } \\
\hat{F}\end{array}$ & $\underset{\hat{N}}{\hat{\alpha}}$ & $\begin{array}{l}\hat{0} \\
\text { के } \\
\text { fे }\end{array}$ & $\begin{array}{l}\text { तै } \\
\text { ळे } \\
\text { హे }\end{array}$ & 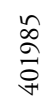 & $\begin{array}{l}\stackrel{+}{n} \\
\stackrel{0}{a}\end{array}$ & 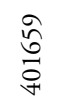 & $\begin{array}{l}\hat{\hat{N}} \\
\stackrel{n}{a} \\
\stackrel{a}{+}\end{array}$ & : \\
\hline Coordinate (Koordinate) GK X (D-48) & $\mathrm{m}$ & $\begin{array}{l}\underset{\hat{N}}{\hat{n}} \\
\hat{n} \\
\hat{n}\end{array}$ & $\begin{array}{l}\stackrel{0}{0} \\
\stackrel{n}{n} \\
\hat{n} \\
\hat{n}\end{array}$ & 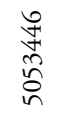 & $\begin{array}{l}\stackrel{n}{f} \\
\stackrel{n}{n} \\
\stackrel{2}{n}\end{array}$ & 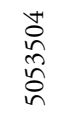 & 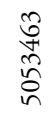 & 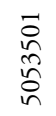 & 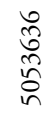 & 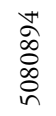 & $\begin{array}{l}\tilde{N} \\
\hat{\sigma} \\
\dot{\infty} \\
\stackrel{0}{n}\end{array}$ & 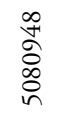 & $\begin{array}{l}\stackrel{+}{\sigma} \\
\stackrel{\sigma}{\circ} \\
\stackrel{0}{\circ} \\
\stackrel{n}{n}\end{array}$ & $\hat{s}$ \\
\hline
\end{tabular}

Diagnostic species of the association (Diagnostične vrste asociacije)

Pr. Fr.

TA Tilia platyphyllos

TA Tilia platyphyllos

TA Tilia platyphyllos

TA Tilia platyphyllos

TA Tilia platyphyllos

QP Cnidium silaifolium

QP Helleborus multifidus subsp. istriacus*

TG Paeonia officinalis

Differential species of the subassociations (Razlikovalnice subasociacij)

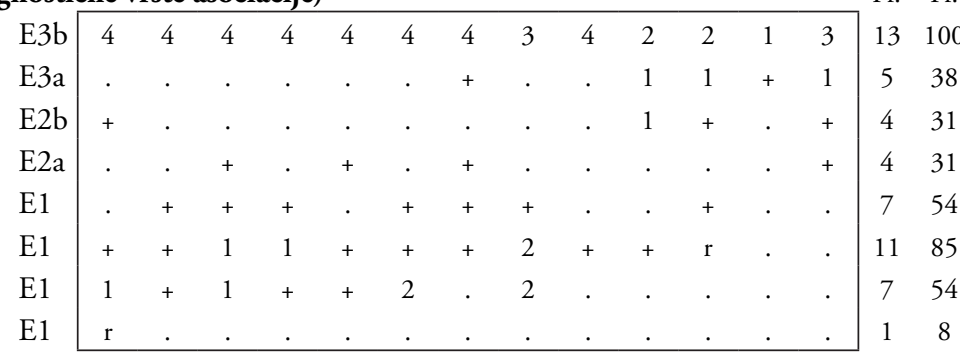

\section{MuA Saxifraga rotundifolia \\ CF Pseudofumaria alba \\ FS Galeobdolon montanum \\ QP Euonymus verrucosa \\ QF Hedera helix}

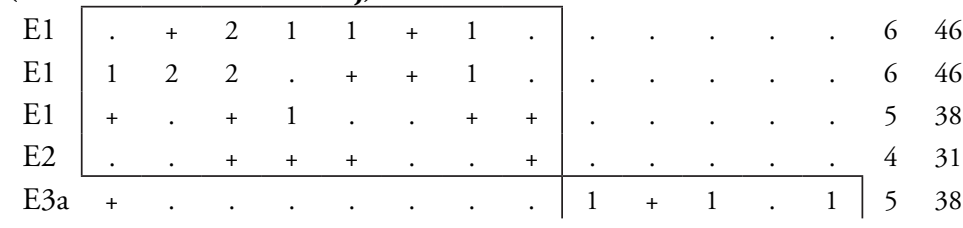




\begin{tabular}{|c|c|c|c|c|c|c|c|c|c|c|c|c|c|c|c|c|c|}
\hline & \multicolumn{2}{|c|}{ Number of relevé (Zaporedna številka popisa) } & 1 & 2 & 3 & 4 & 5 & 6 & 7 & 8 & 9 & 10 & 11 & 12 & 13 & Pr. & Fr. \\
\hline $\mathrm{QF}$ & Hedera helix & E1 & . & + & . & . & . & . & . & . & 1 & 1 & 1 & 1 & 1 & 6 & 46 \\
\hline QP & Cornus mas & $\mathrm{E} 2 \mathrm{~b}$ & . & . & . & + & . & . & . & . & 1 & + & + & 1 & + & 6 & 46 \\
\hline QR & Castanea sativa & $\mathrm{E} 3 \mathrm{~b}$ & . & . & . & . & . & . & . & . & + & + & $\mathrm{r}$ & + & + & 5 & 38 \\
\hline $\mathrm{CO}$ & Ruscus aculeatus & E2a & . & . & . & . & . & . & . & . & 2 & . & 3 & 1 & 2 & 4 & 31 \\
\hline \multirow[t]{23}{*}{$\mathrm{Ta}$} & \multicolumn{17}{|l|}{ Tilio-Acerion } \\
\hline & Acer pseudoplatanus & $\mathrm{E} 3 \mathrm{~b}$ & + & . & . & . & . & + & $\mathrm{r}$ & . & + & . & . & + & + & 6 & 46 \\
\hline & Acer pseudoplatanus & E3a & . & . & . & . & . & + & . & . & . & . & . & + & . & 2 & 15 \\
\hline & Acer pseudoplatanus & $\mathrm{E} 2 \mathrm{~b}$ & . & . & . & . & + & + & + & . & . & . & . & . & + & 4 & 31 \\
\hline & Acer pseudoplatanus & E2a & + & . & + & . & + & + & . & . & . & . & . & + & + & 6 & 46 \\
\hline & Acer pseudoplatanus & E1 & 1 & 1 & 1 & . & + & 1 & 1 & + & 1 & + & . & + & + & 11 & 85 \\
\hline & Adoxa moschatellina & E1 & 1 & + & + & + & . & + & 1 & + & . & . & . & . & . & 7 & 54 \\
\hline & Ulmus glabra & $\mathrm{E} 3 \mathrm{~b}$ & . & + & . & . & . & . & . & . & $\mathrm{r}$ & + & 4 & 3 & 3 & 6 & 46 \\
\hline & Ulmus glabra & E3a & . & . & . & . & . & . & . & . & . & . & 1 & 1 & 1 & 3 & 23 \\
\hline & Ulmus glabra & $\mathrm{E} 2 \mathrm{~b}$ & . & . & . & . & . & . & . & . & + & + & 1 & . & + & 4 & 31 \\
\hline & Ulmus glabra & $\mathrm{E} 2 \mathrm{a}$ & . & + & . & . & . & . & . & . & . & + & . & 1 & 1 & 4 & 31 \\
\hline & Ulmus glabra & E1 & . & + & . & . & . & . & . & . & + & + & + & + & . & 5 & 38 \\
\hline & Geranium robertianum & E1 & 1 & 1 & . & . & + & 1 & 1 & . & . & . & + & . & . & 6 & 46 \\
\hline & Corydalis solida & E1 & . & + & + & 1 & . & + & 1 & . & . & . & . & . & . & 5 & 38 \\
\hline & Thalictrum aquilegiifolium & E1 & + & . & + & + & . & + & . & . & . & . & . & . & . & 4 & 31 \\
\hline & Tilia cordata & E3b & . & . & . & . & . & + & . & . & . & + & + & . & + & 4 & 31 \\
\hline & Tilia cordata & E3a & . & . & . & . & . & . & . & . & . & + & . & . & . & 1 & 8 \\
\hline & Tilia cordata & $\mathrm{E} 2 \mathrm{~b}$ & . & . & . & . & . & . & . & . & . & + & . & . & . & 1 & 8 \\
\hline & Tilia cordata & E2a & . & . & . & . & . & . & . & . & + & . & . & . & . & 1 & 8 \\
\hline & Glechoma hirsuta & E1 & . & . & . & . & . & . & + & . & . & . & . & . & . & 1 & 8 \\
\hline & Acer platanoides & E3b & . & . & . & . & . & . & . & . & . & . & . & + & . & 1 & 8 \\
\hline & Acer platanoides & $\mathrm{E} 1$ & . & . & . & . & . & . & . & . & . & . & . & + & . & 1 & 8 \\
\hline & Aruncus dioicus & E1 & . & . & . & . & . & . & . & . & . & . & . & . & + & 1 & 8 \\
\hline \multirow[t]{8}{*}{$\mathrm{EC}$} & \multicolumn{17}{|l|}{ Erythronio-Carpinion } \\
\hline & Galanthus nivalis & E1 & 1 & 1 & 1 & 1 & 1 & 2 & 1 & 1 & . & . & 3 & 2 & 3 & 11 & 85 \\
\hline & Primula vulgaris & E1 & + & . & . & + & . & + & + & 1 & . & . & . & + & + & 7 & 54 \\
\hline & Erythronium dens-canis & E1 & . & . & 1 & 1 & 1 & 1 & 1 & . & . & . & . & . & . & 5 & 38 \\
\hline & Ornithogalum pyrenaicum & E1 & + & . & + & . & . & . & . & + & . & . & . & . & . & 3 & 23 \\
\hline & Lonicera caprifolium & $\mathrm{E} 2 \mathrm{a}$ & . & . & . & . & . & . & . & . & . & + & . & . & + & 2 & 15 \\
\hline & Crocus vernus subsp. vernus* & E1 & . & . & . & . & + & . & . & . & . & . & . & . & . & 1 & 8 \\
\hline & Helleborus odorus & E1 & . & . & . & . & . & . & . & . & + & . & . & . & . & 1 & 8 \\
\hline \multirow[t]{7}{*}{$\mathrm{AF}$} & \multicolumn{17}{|l|}{ Aremonio-Fagion } \\
\hline & Cyclamen purpurascens & E1 & . & + & 1 & 1 & + & 1 & + & + & 1 & 1 & 1 & + & 2 & 12 & 92 \\
\hline & Lamium orvala & E1 & 2 & 1 & . & 1 & 1 & 2 & 2 & 1 & . & . & 1 & + & 2 & 10 & 77 \\
\hline & Cardamine enneaphyllos & E1 & . & . & 2 & 2 & . & 2 & . & . & 2 & 2 & 3 & 2 & 3 & 8 & 62 \\
\hline & Geranium nodosum & E1 & + & 1 & + & 1 & 1 & 1 & + & . & . & . & . & . & . & 7 & 54 \\
\hline & Calamintha grandiflora & E1 & . & . & + & 1 & 1 & 1 & + & . & . & . & . & . & . & 5 & 38 \\
\hline & Hacquetia epipactis & E1 & . & . & . & . & . & . & . & . & + & . & . & + & + & 3 & 23 \\
\hline \multirow[t]{3}{*}{ AI } & \multicolumn{17}{|l|}{ Alnion incanae } \\
\hline & Rubus caesius & E1 & . & . & . & . & + & . & . & . & . & + & . & . & . & 2 & 15 \\
\hline & Aesculus hippocastanum & E3b & $\mathrm{r}$ & . & . & . & . & . & . & . & . & . & . & . & . & 1 & 8 \\
\hline \multirow[t]{5}{*}{ FS } & \multicolumn{17}{|l|}{ Fagetalia sylvaticae } \\
\hline & Asarum europaeum subsp. caucasicum & $\mathrm{E} 1$ & 1 & 1 & + & + & 1 & + & 1 & + & + & . & + & + & + & 12 & 92 \\
\hline & Salvia glutinosa & E1 & + & + & + & + & + & + & + & + & + & + & . & + & + & 12 & 92 \\
\hline & Mercurialis perennis & E1 & . & + & 1 & 1 & 1 & 2 & 2 & + & + & + & 2 & 3 & 2 & 12 & 92 \\
\hline & Mycelis muralis & E1 & 1 & + & + & + & + & + & + & . & . & + & + & + & + & 11 & 85 \\
\hline
\end{tabular}




\begin{tabular}{|c|c|c|c|c|c|c|c|c|c|c|c|c|c|c|c|c|c|}
\hline & Number of relevé (Zaporedna številka popisa & & 1 & 2 & 3 & 4 & 5 & 6 & 7 & 8 & 9 & 10 & 11 & 12 & 13 & Pr. & Fr. \\
\hline & Lathyrus vernus & E1 & + & . & 1 & 1 & 1 & 1 & 1 & . & + & 2 & + & + & + & 11 & 85 \\
\hline & Dryopteris filix-mas & E1 & + & + & + & 1 & 1 & 1 & 1 & . & + & . & . & + & + & 10 & 77 \\
\hline & Campanula trachelium & E1 & . & . & + & 1 & 1 & + & 1 & . & . & + & + & + & + & 9 & 69 \\
\hline & Polygonatum multiflorum & E1 & . & . & + & . & 1 & 1 & + & + & . & . & + & + & 1 & 8 & 62 \\
\hline & Heracleum sphondylium & E1 & 1 & + & + & + & 1 & + & + & + & . & . & . & . & . & 8 & 62 \\
\hline & Symphytum tuberosum & E1 & + & + & . & . & + & + & . & + & . & + & . & + & + & 8 & 62 \\
\hline & Pulmonaria officinalis & E1 & + & + & . & + & . & + & + & + & . & . & . & . & + & 7 & 54 \\
\hline & Euphorbia dulcis & E1 & + & . & + & + & 1 & + & . & . & . & + & . & + & . & 7 & 54 \\
\hline & Actaea spicata & E1 & + & + & . & . & + & 1 & + & . & . & . & . & . & . & 5 & 38 \\
\hline & Galeobdolon flavidum & E1 & . & . & . & . & . & . & . & . & + & . & + & + & + & 4 & 31 \\
\hline & Fraxinus excelsior & $\mathrm{E} 3 \mathrm{~b}$ & + & 1 & . & . & + & . & 1 & . & . & . & . & . & . & 4 & 31 \\
\hline & Fraxinus excelsior & $\mathrm{E} 2 \mathrm{a}$ & . & . & . & . & . & . & . & . & . & . & . & + & + & 2 & 15 \\
\hline & Fraxinus excelsior & E1 & + & 1 & . & . & . & . & + & . & . & . & . & . & . & 3 & 23 \\
\hline & Fagus sylvatica & $\mathrm{E} 3 \mathrm{~b}$ & . & . & . & . & . & . & $\mathrm{r}$ & . & . & 1 & + & . & $\mathrm{r}$ & 4 & 31 \\
\hline & Fagus sylvatica & E3a & . & . & . & . & . & . & . & . & . & + & + & . & . & 2 & 15 \\
\hline & Fagus sylvatica & E1 & . & . & $\mathrm{r}$ & . & . & . & . & . & . & . & . & . & . & 1 & 8 \\
\hline & Carpinus betulus & $\mathrm{E} 3 \mathrm{~b}$ & . & + & . & + & . & . & . & . & . & . & . & . & . & 2 & 15 \\
\hline & Carpinus betulus & E3a & . & . & + & . & . & + & + & . & . & . & . & . & . & 3 & 23 \\
\hline & Carpinus betulus & $\mathrm{E} 2 \mathrm{~b}$ & . & . & . & + & . & . & . & . & . & . & . & . & . & 1 & 8 \\
\hline & Sambucus nigra & $\mathrm{E} 2 \mathrm{~b}$ & + & . & . & . & . & . & . & . & . & . & . & + & + & 3 & 23 \\
\hline & Sambucus nigra & E2a & + & . & . & . & . & . & + & . & . & . & . & . & . & 2 & 15 \\
\hline & Scrophularia nodosa & E1 & . & + & . & . & + & . & + & . & . & . & . & . & . & 3 & 23 \\
\hline & Lilium martagon & E1 & . & . & . & . & . & . & . & . & + & . & . & + & + & 3 & 23 \\
\hline & Corydalis cava & E1 & . & + & . & . & . & . & + & . & . & . & . & . & . & 2 & 15 \\
\hline & Paris quadrifolia & E1 & . & . & . & . & . & + & + & . & . & . & . & . & . & 2 & 15 \\
\hline & Galium laevigatum & E1 & . & . & . & . & . & . & . & . & . & . & . & + & + & 2 & 15 \\
\hline & Ranunculus lanuginosus & E1 & . & + & . & . & . & + & . & . & . & . & . & . & . & 2 & 15 \\
\hline & Prunus avium & $\mathrm{E} 3 \mathrm{~b}$ & . & . & . & . & $\mathrm{r}$ & . & $\mathrm{r}$ & . & . & . & . & . & . & 2 & 15 \\
\hline & Prunus avium & E1 & . & . & . & . & . & + & . & . & . & . & . & . & . & 1 & 8 \\
\hline & Melica nutans & E1 & . & . & . & . & . & + & . & + & . & . & . & . & . & 2 & 15 \\
\hline & Prenanthes purpurea & E1 & . & . & . & . & . & . & . & . & . & + & . & . & . & 1 & 8 \\
\hline & Laburnum alpinum & E3a & . & . & . & . & . & . & . & . & . & . & . & + & . & 1 & 8 \\
\hline & Laburnum alpinum & $\mathrm{E} 2 \mathrm{~b}$ & . & . & . & . & . & . & . & . & . & + & . & . & . & 1 & 8 \\
\hline & Laburnum alpinum & $\mathrm{E} 2 \mathrm{a}$ & . & . & . & . & . & . & . & . & . & . & + & . & . & 1 & 8 \\
\hline & Laburnum alpinum & E1 & . & . & . & . & . & . & . & . & . & + & . & . & . & 1 & 8 \\
\hline & Cardamine bulbifera & E1 & . & . & . & . & . & . & . & . & . & . & . & . & + & 1 & 8 \\
\hline \multirow[t]{3}{*}{$\mathrm{CO}$} & Carpinion orientalis & & & & & & & & & & & & & & & & \\
\hline & Sesleria autumnalis & E1 & + & + & 3 & 3 & 2 & 1 & + & 3 & 1 & 2 & + & + & + & 13 & 100 \\
\hline & Acer monspessulanum & $\mathrm{E} 3 \mathrm{~b}$ & . & . & . & . & . & . & . & . & . & + & . & . & . & 1 & 8 \\
\hline \multirow[t]{11}{*}{ QP } & Quercetalia pubescenti-petraeae & & & & & & & & & & & & & & & & \\
\hline & Lathyrus venetus & E1 & 1 & 1 & + & + & . & + & + & . & + & + & + & + & + & 11 & 85 \\
\hline & Ostrya carpinifolia & $\mathrm{E} 3 \mathrm{~b}$ & . & . & + & $\mathrm{r}$ & 1 & . & . & . & 1 & 3 & 2 & 2 & + & 8 & 62 \\
\hline & Ostrya carpinifolia & E3a & . & . & + & + & . & . & . & . & . & + & . & . & . & 3 & 23 \\
\hline & Fraxinus ornus & $\mathrm{E} 3 \mathrm{~b}$ & + & . & . & 1 & 1 & . & . & 1 & + & . & . & + & + & 7 & 54 \\
\hline & Fraxinus ornus & E3a & + & + & 1 & + & . & + & + & . & + & 1 & . & . & . & 8 & 62 \\
\hline & Fraxinus ornus & $\mathrm{E} 2 \mathrm{~b}$ & . & . & . & + & + & . & . & . & . & . & . & . & . & 2 & 15 \\
\hline & Fraxinus ornus & E2a & . & . & . & 1 & + & . & . & . & . & + & . & . & . & 3 & 23 \\
\hline & Fraxinus ornus & E1 & + & . & + & . & . & . & . & . & + & . & . & . & . & 3 & 23 \\
\hline & Tanacetum corymbosum & E1 & + & . & + & 1 & + & + & + & 1 & . & + & . & . & . & 8 & 62 \\
\hline & Convallaria majalis & E1 & + & . & 1 & 1 & 2 & + & . & . & . & + & . & . & . & 6 & 46 \\
\hline
\end{tabular}




\begin{tabular}{|c|c|c|c|c|c|c|c|c|c|c|c|c|c|c|c|c|c|}
\hline & \multicolumn{2}{|c|}{ Number of relevé (Zaporedna številka popisa) } & 1 & 2 & 3 & 4 & 5 & 6 & 7 & 8 & 9 & 10 & 11 & 12 & 13 & Pr. & Fr. \\
\hline & Melittis melissophyllum & E1 & + & $\cdot$ & + & + & + & + & . & 1 & . & . & . & . & . & 6 & 46 \\
\hline & Mercurialis ovata & E1 & 1 & . & 1 & + & . & . & + & + & . & . & . & . & . & 5 & 38 \\
\hline & Sorbus aria (Aria edulis) & $\mathrm{E} 3 \mathrm{~b}$ & + & . & . & + & + & . & . & 1 & . & . & . & . & . & 4 & 3 \\
\hline & Sorbus aria (Aria edulis) & E3a & . & . & + & . & + & + & . & . & + & + & . & . & . & 5 & 38 \\
\hline & Sorbus aria (Aria edulis) & $\mathrm{E} 2 \mathrm{~b}$ & . & . & . & . & + & . & . & . & . & + & . & . & . & 2 & 15 \\
\hline & Sorbus aria (Aria edulis) & $\mathrm{E} 2 \mathrm{a}$ & . & . & + & . & . & . & . & . & . & . & . & . & . & 1 & 8 \\
\hline & Sorbus aria (Aria edulis) & E1 & . & + & . & . & . & . & . & . & . & . & . & . & . & 1 & 8 \\
\hline & Asparagus tenuifolius & E1 & + & . & + & + & . & . & . & + & . & . & . & . & . & 4 & 31 \\
\hline & Campanula persicifolia & E1 & . & . & + & + & + & . & . & + & . & . & . & . & . & 4 & 31 \\
\hline & Quercus cerris & E3b & + & . & . & $\mathrm{r}$ & . & . & . & 3 & . & . & . & . & . & 3 & 23 \\
\hline & Mercurialis $x$ paxii & E1 & . & . & + & . & . & . & + & . & . & . & . & . & . & 2 & 1 \\
\hline & Arabis turrita & E1 & . & . & . & . & . & . & . & . & . & . & + & + & . & 2 & 1 \\
\hline & Hypericum montanum & E1 & . & . & + & . & . & . & . & . & . & . & . & . & . & 1 & 8 \\
\hline & Lathyrus niger & E1 & . & . & . & . & . & . & . & + & . & . & . & . & . & 1 & 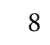 \\
\hline & Orchis pallens & E1 & . & . & . & . & . & . & . & . & $\mathrm{r}$ & . & . & . & . & 1 & 8 \\
\hline & Tamus communis & E1 & . & . & . & . & . & . & . & . & . & . & . & . & + & 1 & 8 \\
\hline & Quercus pubescens & E3b & . & . & . & . & . & . & . & . & . & . & . & . & $\mathrm{r}$ & 1 & 8 \\
\hline QR & Quercetalia roboris & & & & & & & & & & & & & & & & \\
\hline & Serratula tinctoria & E1 & . & . & . & . & . & . & . & . & . & + & . & . & . & 1 & 8 \\
\hline QF & Querco-Fagetea & & & & & & & & & & & & & & & & \\
\hline & Corylus avellana & E2b & 1 & 1 & . & 1 & 1 & + & 1 & 1 & + & 1 & 1 & 2 & 2 & 12 & 92 \\
\hline & Corylus avellana & E2a & . & . & + & $\cdot$ & . & . & . & . & . & . & . & 1 & 1 & 3 & 23 \\
\hline & Hepatica nobilis & E1 & . & + & 1 & + & 1 & 1 & 1 & 1 & . & 1 & + & + & 2 & 11 & 85 \\
\hline & Lonicera xylosteum & E2 & . & + & + & + & 1 & 1 & + & 1 & . & . & . & . & . & 7 & 54 \\
\hline & Acer campestre & E3b & . & . & . & . & . & . & . & . & . & . & + & . & + & 2 & 15 \\
\hline & Acer campestre & E3a & + & + & . & + & + & + & . & + & . & . & . & . & . & 6 & 46 \\
\hline & Acer campestre & $\mathrm{E} 2 \mathrm{~b}$ & + & . & . & . & . & + & . & . & . & . & . & . & . & 2 & 1 \\
\hline & Acer campestre & E2a & . & . & + & . & + & + & . & + & . & . & . & . & . & 4 & 3 \\
\hline & Acer campestre & E1 & . & + & + & + & . & . & + & + & . & + & . & + & . & 7 & 5 \\
\hline & Veratrum nigrum & E1 & . & . & . & . & 1 & $\mathrm{r}$ & + & . & 1 & + & . & + & . & 6 & 46 \\
\hline & Scilla bifolia & E1 & . & + & 1 & + & + & 1 & + & . & . & . & . & . & . & 6 & 4 \\
\hline & Clematis vitalba & E3a & . & . & . & . & . & . & . & . & + & + & . & . & $\mathrm{r}$ & 3 & 23 \\
\hline & Clematis vitalba & E2a & 1 & + & + & + & . & . & . & + & . & . & . & . & . & 5 & 38 \\
\hline & Clematis vitalba & E1 & . & . & . & . & . & + & . & . & . & + & . & + & . & 3 & 2 \\
\hline & Melica uniflora & E1 & 1 & + & . & . & . & . & + & 2 & . & . & . & . & . & 4 & 3 \\
\hline & Carex digitata & E1 & . & . & + & + & + & . & + & . & . & . & . & . & . & 4 & 3 \\
\hline & Aegopodium podagraria & E1 & . & + & . & . & . & + & + & . & . & . & . & . & . & 3 & 23 \\
\hline & Moehringia trinervia & E1 & . & + & . & + & . & . & . & . & . & . & . & . & . & 2 & 1 \\
\hline & Anemone nemorosa & E1 & . & . & . & . & . & . & . & . & + & . & . & . & 2 & 2 & 1 \\
\hline & Quercus petraea & E3b & . & . & . & . & . & . & . & . & . & + & 1 & . & . & 2 & 15 \\
\hline & Quercus petraea & E1 & . & . & . & . & . & . & . & . & . & . & + & . & . & 1 & 8 \\
\hline & Crataegus laevigata & E2a & . & . & . & . & . & . & . & . & . & . & . & + & + & 2 & 15 \\
\hline & Malus sylvestris & E3a & $\mathrm{r}$ & . & . & . & . & . & . & . & . & . & . & . & . & 1 & \\
\hline & Ranunculus ficaria & E1 & . & + & . & . & . & . & . & . & . & . & . & . & . & 1 & \\
\hline & Pyrus pyraster & E2a & . & 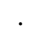 & + & . & . & . & . & . & . & . & $\cdot$ & $\cdot$ & . & 1 & 8 \\
\hline & Rosa arvensis & $\mathrm{E} 2 \mathrm{a}$ & . & . & . & . & . & . & . & 1 & . & . & . & . & . & 1 & 8 \\
\hline & Spiraea chamaedryfolia & E2a & . & · & . & . & . & . & . & . & . & . & + & . & . & 1 & 8 \\
\hline & Lathraea squamaria & E1 & . & . & . & . & . & . & . & . & . & . & . & . & + & 1 & 8 \\
\hline $\mathrm{VP}$ & Vaccinio-Piceetea & & & & & & & & & & & & & & & & \\
\hline & Solidago virgaurea & E1 & . & + & + & + & + & + & . & . & . & . & . & . & . & 5 & \\
\hline
\end{tabular}




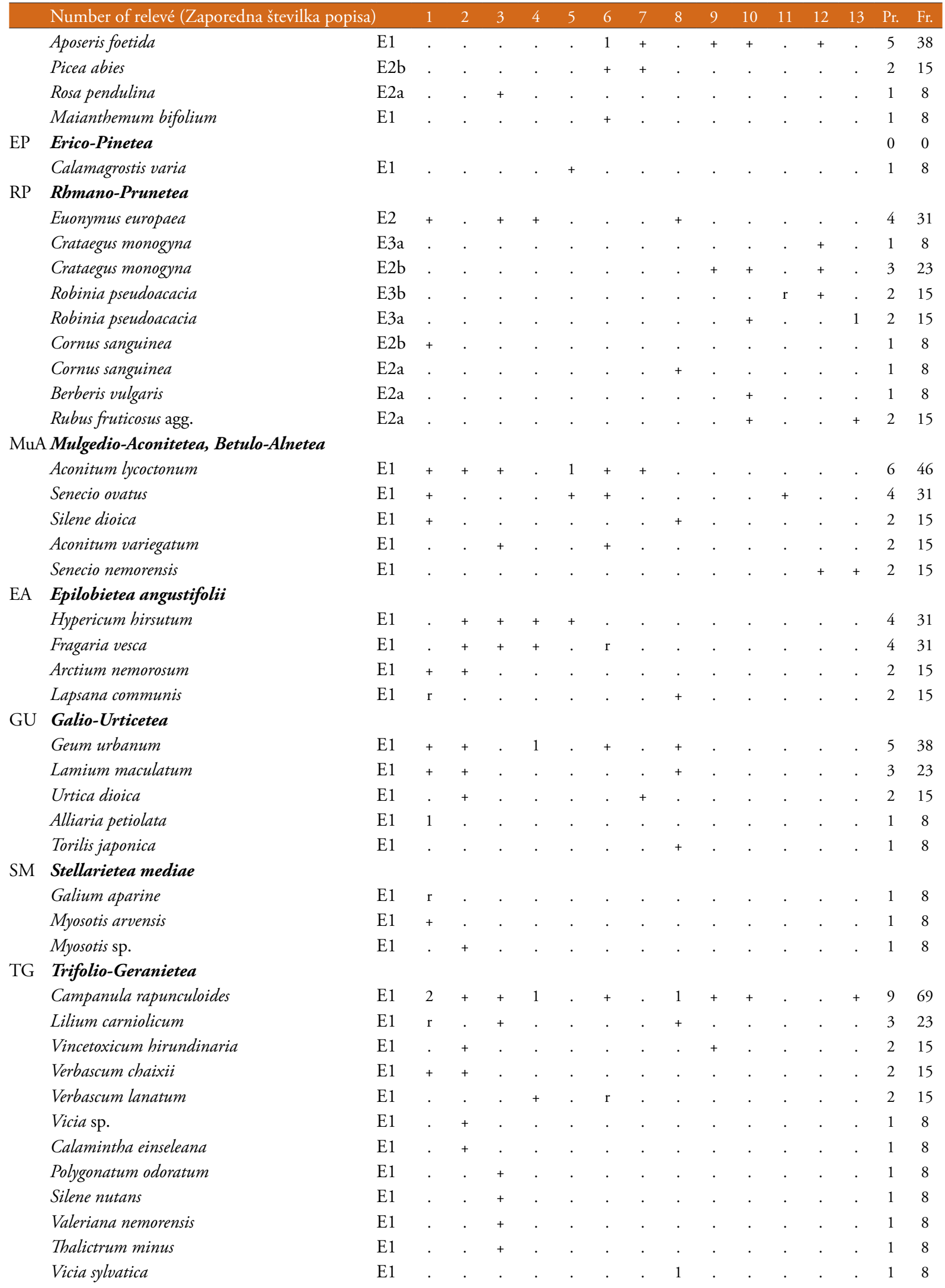




\section{$\begin{array}{lllllllllllllllll}\text { Number of relevé (Zaporedna šsevilka popisa) } & 1 & 2 & 3 & 4 & 5 & 6 & 7 & 8 & 9 & 10 & 11 & 12 & 13 & \text { Pr. } & \text { Fr. }\end{array}$}

FB Festuco-Brometea

Taraxacum laevigatum

E1

Arabis hirsuta

Brachypodium rupestre

E1

E1

KC Koelerio-Corynephoretea

Cardaminopsis arenosa

E1

MA Molinio-Arrhenatheretea

Veronica chamaedrys

Taraxacum sect. Ruderalia

$\mathrm{E} 1++++.+1 .+2.754$

Cy Cystopteridion fragilis

Moehringia muscosa

$\mathrm{E} 1++.+.+.+.+2.431$

Cystopteris fragilis

$\mathrm{E} 1$. $1+++1 .+1 .+862$

E1

PS Physoplexido-Saxifragion, Potentilletalia caulescentis

Campanula pyramidalis

AT Asplenietea trichomanis

Asplenium trichomanes

Sedum maximum

Ceterach officinarum

Asplenium ruta-muraria

Polypodium vulgare

$\mathrm{E} 1+++\ldots+.+. \quad$. . . . 538

ML Mosses and lichens (Mahovi in lišaji)

Neckera crispa

Homalothecium lutescens

Schistidium apocarpum

Ctenidium molluscum

Anomodon viticulosus

Isothecium alopecuroides

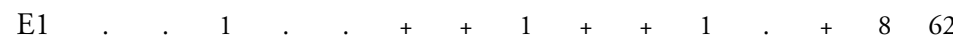

Neckera complanata

Porella platyphylla

Plagiochila porelloides

Peltigera canina

Polytrichum formosum

Homalothecium sericeum

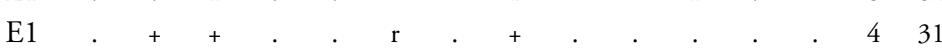

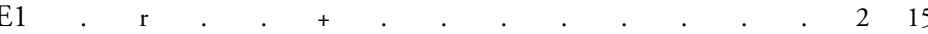

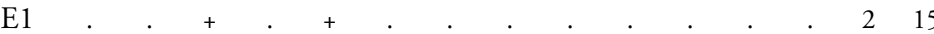

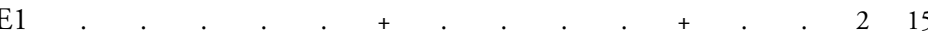

Mnium sp.

\begin{tabular}{|c|c|c|c|c|c|c|c|c|c|c|c|c|c|c|c|}
\hline E0 & & & 2 & 1 & 1 & 2 & r & & 1 & 2 & 2 & + & + & 9 & 69 \\
\hline E0 & 2 & 2 & 1 & 2 & 1 & 1 & 2 & & . & . & 1 & . & . & 8 & 62 \\
\hline E0 & 1 & + & + & . & 1 & 1 & . & & 1 & + & + & . & . & 8 & 62 \\
\hline E0 & . & . & 2 & 1 & 1 & 2 & 1 & . & 1 & 2 & . & . & + & 8 & 62 \\
\hline E0 & 1 & . & 1 & 1 & . & 1 & . & 1 & . & • & 1 & . & + & 7 & 54 \\
\hline E0 & . & . & . & . & . & 1 & 1 & & 1 & r & 2 & 1 & 2 & 6 & 46 \\
\hline E0 & . & . & . & + & + & . & + & r & . & $\cdot$ & 1 & $\cdot$ & . & 4 & 31 \\
\hline E0 & . & + & . & · & . & 1 & . & & . & • & . & . & . & 2 & 15 \\
\hline E0 & . & . & + & . & . & . & + & 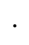 & . & . & . & . & . & 2 & 15 \\
\hline E0 & . & $\cdot$ & . & . & . & + & + & . & . & . & . & . & . & 2 & 15 \\
\hline E0 & . & . & + & . & . & . & . & & . & & . & . & . & 1 & 8 \\
\hline E0 & . & . & . & . & . & 1 & . & & . & & . & . & . & 1 & 8 \\
\hline E0 & $\cdot$ & . & . & . & . & . & + & & . & . & . & . & . & 1 & 8 \\
\hline
\end{tabular}

\section{Legend - Legenda}

A Limestone - apnenec

Gr Gravel - grušč

L Marlstone-laporovec

Re Rendzina - rendzina

Co Colluvial soil - koluvialna tla

Pr. Presence (number of relevés in which the species is presented) - število popisov, v katerih se pojavlja vrsta

Fr. Frequency in $\%$ - frekvenca $v \%$

Helleborus multifidus subsp. istriacus* (incl. Helleborus dumetorum subsp. istriacus) - Rottensteiner (2016)

Crocus vernus subsp. vernus* is according to new findings Crocus heuffellianus Herb. - Peruzzi (2016) 
Table 4 (Tabela 4): Paeonio officinalis-Tilietum platyphylli hierochloetosum australis.

\begin{tabular}{|c|c|c|c|c|c|c|c|c|c|}
\hline Number of relevé (Zaporedna številka popisa) & & 1 & 2 & 3 & 4 & 5 & 6 & 7 & 8 \\
\hline $\begin{array}{l}\text { Database number of relevé } \\
\text { (Delovna številka popisa) }\end{array}$ & & 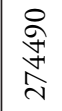 & 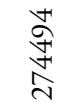 & 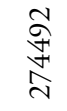 & 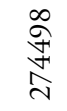 & 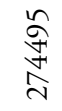 & 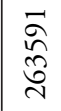 & 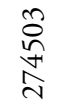 & 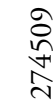 \\
\hline Elevation in $\mathrm{m}$ (Nadmorska višina $\mathrm{v} \mathrm{m})$ & & 320 & 340 & 280 & 330 & 340 & 765 & 320 & 280 \\
\hline Aspect (Lega) & & $\mathrm{N}$ & $\mathrm{N}$ & NNE & $\mathrm{NE}$ & $\mathrm{NE}$ & W & $\mathrm{W}$ & $S$ \\
\hline Slope in degrees (Nagib v stopinjah) & & 30 & $35-45$ & 30 & 30 & 50 & 35 & 30 & 2 \\
\hline Parent material (Matična podlaga) & & Gr & A & $\mathrm{Gr}$ & $\mathrm{Gr}$ & A & A & $\mathrm{Gr}$ & A \\
\hline Soil (Tla) & & $\operatorname{Re}$ & $\operatorname{Re}$ & $\operatorname{Re}$ & $\operatorname{Re}$ & $\operatorname{Re}$ & $\operatorname{Re}$ & $\mathrm{Rj}$ & $\mathrm{Rj}$ \\
\hline Stoniness in \% (Kamnitost v \%) & & 20 & 40 & 20 & 20 & 80 & 50 & 10 & , \\
\hline \multicolumn{10}{|l|}{ Cover in \% (Zastiranje v \%): } \\
\hline Upper tree layer (Zgornja drevesna plast) & $\mathrm{E} 3 \mathrm{~b}$ & 70 & 60 & 70 & 90 & 20 & 80 & 70 & 80 \\
\hline Lower tree layer (Spodnja drevesna plasti) & E3a & 20 & 20 & 30 & . & . & . & 20 & . \\
\hline Shrub layer (Grmovna plast) & E2 & 20 & 20 & 10 & 10 & 70 & 20 & 20 & 5 \\
\hline Herb layer (Zeliščna plast) & E1 & 90 & 70 & 70 & 90 & 50 & 70 & 80 & 30 \\
\hline Moss layer (Mahovna plast) & E0 & 20 & 30 & 40 & 20 & 69 & 30 & 10 & \\
\hline Maximum tree diameter (Maksimalni premer dreves) & $\mathrm{cm}$ & 45 & 35 & 35 & 25 & 20 & 25 & 35 & 35 \\
\hline Maximum tree height (Maksimalna višina dreves) & $\mathrm{m}$ & 19 & 18 & 20 & 18 & 10 & 10 & 20 & 20 \\
\hline Number of species (Število vrst) & & 47 & 51 & 60 & 47 & 62 & 62 & 63 & 34 \\
\hline Relevé area (Velikost popisne ploskve) & $\mathrm{m}^{2}$ & 400 & 400 & 400 & 400 & 400 & 200 & 400 & 400 \\
\hline Date of taking relevé (Datum popisa) & & 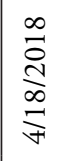 & $\underset{\underset{\forall}{\infty}}{\stackrel{\infty}{\stackrel{\infty}{0}}}$ & $\frac{\infty}{\stackrel{\infty}{ٍ}}$ & 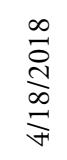 & 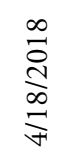 & 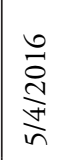 & $\frac{\underset{\infty}{\stackrel{\infty}{d}}}{\stackrel{\infty}{\leftrightarrows}}$ & \\
\hline Locality (Nahajališče) & & 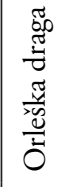 & 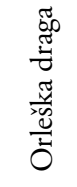 & 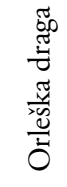 & 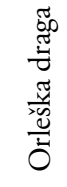 & 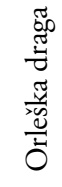 & 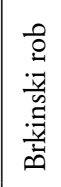 & 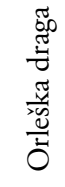 & \\
\hline Quadrant (Kvadrant) & & 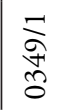 & $\underset{\text { ఠે }}{\stackrel{a}{\sigma}}$ & 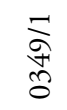 & 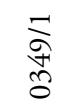 & $\underset{\text { సे }}{\vec{a}}$ & $\stackrel{n}{\tilde{n}}$ & 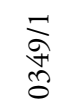 & \\
\hline Coordinate (Koordinate) GK Y (D-48) & $\mathrm{m}$ & 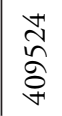 & 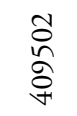 & 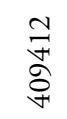 & 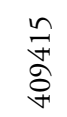 & $\begin{array}{l}\stackrel{0}{+} \\
\stackrel{+}{\circ} \\
\stackrel{+}{+}\end{array}$ & $\begin{array}{l}8 \\
\stackrel{\circ}{\circ} \\
\stackrel{\leftrightarrow}{+}\end{array}$ & $\begin{array}{l}n \\
\hat{n} \\
\hat{\sigma} \\
\hat{+}\end{array}$ & \\
\hline Coordinate (Koordinate) GK X (D-48) & $\mathrm{m}$ & 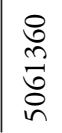 & 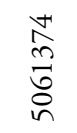 & 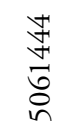 & 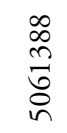 & $\begin{array}{l}\vec{n} \\
\stackrel{\circ}{\circ}\end{array}$ & 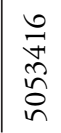 & $\begin{array}{l}\stackrel{n}{+} \\
\underset{\delta}{\circ} \\
\stackrel{n}{n}\end{array}$ & \\
\hline
\end{tabular}

\section{Diagnostic species of the association (Diagnostične vrste asociacije)}

Pr. Fr.

$1-5 \quad 1-5$

TA Tilia platyphyllos

TA Tilia platyphyllos

TA Tilia platyphyllos

TA Tilia platyphyllos

QP Helleborus multifidus subsp. istriacus*

TG Paeonia officinalis

\begin{tabular}{l|ccccc|ccccc} 
E3b & 1 & 1 & 2 & 2 & $\cdot$ & $\cdot$ &. &. & 4 & 80 \\
E3a & + & + & 1 & + & 1 & $\cdot$ &. &. & 5 & 100 \\
E2b & $\cdot$ & $\cdot$ & 1 & $\cdot$ & + & + &. &. & 2 & 40 \\
E2a & $\cdot$ &. & + & + & $\cdot$ & $\cdot$ & + &. & 2 & 40 \\
E1 & 1 & + & + & $\cdot$ & + & + & 1 & 1 & 4 & 80 \\
E1 & 1 &. &. &. & + & 1 &. &. & 2 & 40
\end{tabular}

Differential species of the subassociation (Razlikovalnice subasociacije)

ML Plagiomnium undulatum

QP Hierochloë australis

FB Sesleria tenuifolia

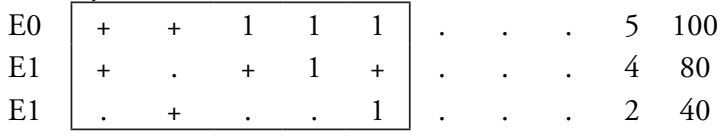


TA Tilio-Acerion

Acer pseudoplatanus

Acer pseudoplatanus

Acer pseudoplatanus

Acer pseudoplatanus

Geranium robertianum

Aruncus dioicus

Tilia cordata

Tilia cordata

Tilia cordata

Isopyrum thalictroides

Adoxa moschatellina

Ulmus glabra

Ulmus glabra

Phyllitis scolopendrium

Acer platanoides

Acer platanoides

Acer platanoides

Thalictrum aquilegiifolium

EC Erythronio-Carpinion

Galanthus nivalis

Primula vulgaris

Erythronium dens-canis

AF Aremonio-Fagion

Cyclamen purpurascens

Cardamine enneaphyllos

Lamium orvala

AI Alnion incanae

Aesculus hippocastanum

FS Fagetalia sylvaticae

Galium laevigatum

Polygonatum multiflorum

Lathyrus vernus

Asarum europaeum subsp. caucasicum

Mycelis muralis

Campanula trachelium

Carpinus betulus

Carpinus betulus

Carpinus betulus

Carpinus betulus

Galeobdolon flavidum

Mercurialis perennis

Galeobdolon montanum

Epipactis helleborine

Salvia glutinosa

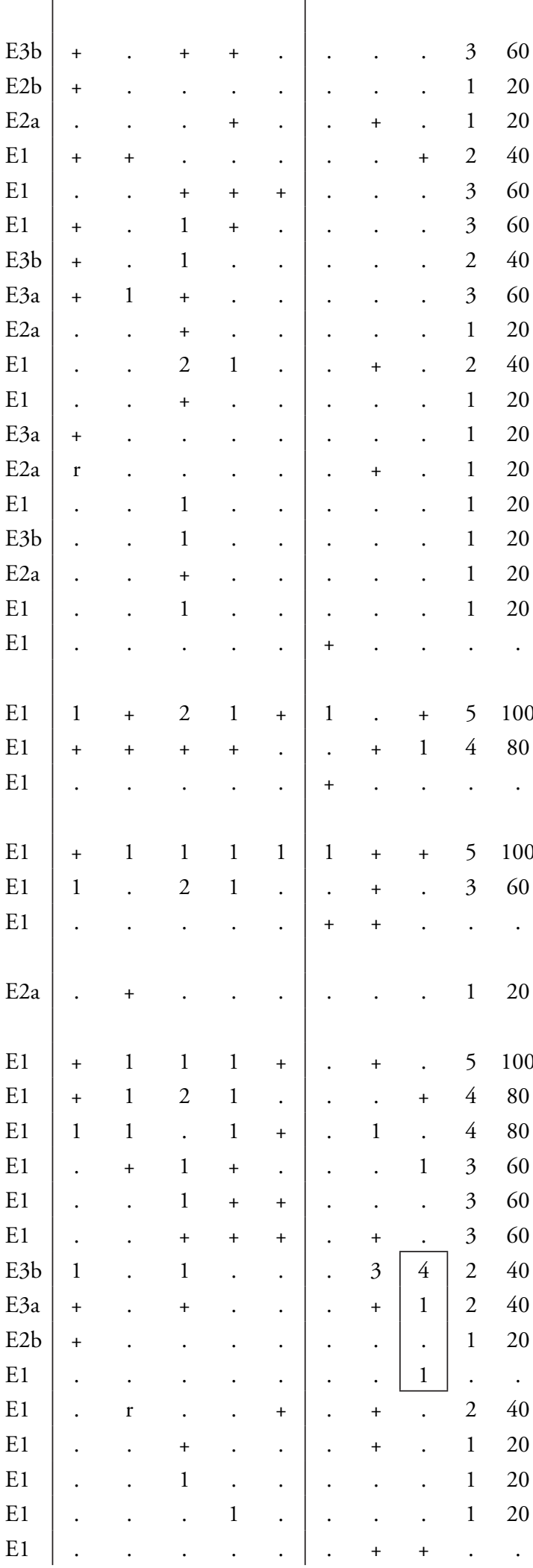




\begin{tabular}{|c|c|c|c|c|c|c|c|c|c|c|c|}
\hline \multicolumn{2}{|c|}{ Number of relevé (Zaporedna številka popisa) } & 1 & \multirow[t]{2}{*}{2} & \multirow{2}{*}{$\begin{array}{l}3 \\
.\end{array}$} & \multirow{2}{*}{$\begin{array}{l}4 \\
.\end{array}$} & \multirow{2}{*}{5} & \multirow{2}{*}{6} & 7 & \multirow{2}{*}{$\begin{array}{l}8 \\
\mathrm{r}\end{array}$} & \multirow{2}{*}{$\begin{array}{l}\text { Pr. } \\
.\end{array}$} & \multirow{2}{*}{ Fr. } \\
\hline Prunus avium & E3b & . & & & & & & . & & & \\
\hline Prunus avium & E1 & . & . & . & . & . & . & + & + & . & . \\
\hline Symphytum tuberosum & E1 & . & . & . & . & . & . & + & 1 & . & . \\
\hline Neottia nidus-avis & E1 & . & . & . & . & . & . & + & . & . & . \\
\hline Sanicula europaea & E1 & . & . & . & . & . & . & + & . & . & . \\
\hline Viola reichenbachiana & E1 & . & . & . & . & . & . & + & . & . & . \\
\hline Corydalis cava & E1 & . & . & . & . & . & . & . & $\mathrm{r}$ & . & . \\
\hline Actaea spicata & E1 & . & . & . & . & . & . & . & + & . & . \\
\hline \multicolumn{12}{|l|}{ Carpinion orientalis } \\
\hline Sesleria autumnalis & E1 & 4 & 4 & 3 & 4 & 3 & 3 & 4 & 1 & 5 & 100 \\
\hline Acer monspessulanum & $\mathrm{E} 2 \mathrm{a}$ & $\mathrm{r}$ & + & . & . & . & . & + & . & 2 & 40 \\
\hline Acer monspessulanum & E1 & . & . & . & . & . & . & + & . & . & . \\
\hline Frangula rupestris & E2a & . & + & . & . & + & 1 & . & . & 2 & 40 \\
\hline \multicolumn{12}{|c|}{ Quercetalia pubescenti-petraeae } \\
\hline Mercurialis ovata & E1 & 1 & 1 & + & 1 & + & + & + & 1 & 5 & 100 \\
\hline Convallaria majalis & E1 & 1 & 1 & 2 & + & + & . & . & . & 5 & 100 \\
\hline Ostrya carpinifolia & E3b & . & 2 & 3 & 4 & . & 4 & + & . & 3 & 60 \\
\hline Ostrya carpinifolia & E3a & 1 & 1 & 1 & . & + & . & . & . & 4 & 80 \\
\hline Ostrya carpinifolia & $\mathrm{E} 2 \mathrm{~b}$ & & . & + & . & 3 & . & . & . & 2 & 40 \\
\hline Ostrya carpinifolia & $\mathrm{E} 2 \mathrm{a}$ & . & . & . & . & + & . & . & . & 1 & 20 \\
\hline Fraxinus ornus & E3b & . & . & + & 1 & . & 2 & . & . & 2 & 40 \\
\hline Fraxinus ornus & E3a & 1 & 1 & 1 & 1 & . & . & . & + & 4 & 80 \\
\hline Fraxinus ornus & $\mathrm{E} 2 \mathrm{~b}$ & 1 & 1 & . & 1 & 2 & + & 1 & . & 4 & 80 \\
\hline Fraxinus ornus & E2a & + & . & . & + & . & 1 & 2 & . & 2 & 40 \\
\hline Fraxinus ornus & E1 & . & . & . & . & . & . & 1 & + & . & . \\
\hline Melittis melissophyllum & E1 & + & 1 & . & + & . & 1 & + & + & 3 & 60 \\
\hline Euonymus verrucosa & $\mathrm{E} 2 \mathrm{a}$ & . & . & 1 & + & + & + & . & . & 3 & 60 \\
\hline Campanula persicifolia & E1 & . & + & . & + & + & + & . & . & 3 & 60 \\
\hline Cornus mas & $\mathrm{E} 2 \mathrm{~b}$ & + & . & + & . & + & . & . & . & 3 & 60 \\
\hline Sorbus aria (Aria edulis) & $\mathrm{E} 3 \mathrm{~b}$ & . & . & + & + & . & . & . & . & 2 & 40 \\
\hline Sorbus aria (Aria edulis) & E3a & + & 1 & . & 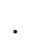 & . & + & + & . & 2 & 40 \\
\hline Sorbus aria (Aria edulis) & $\mathrm{E} 2 \mathrm{~b}$ & + & 1 & . & . & . & . & . & . & 2 & 40 \\
\hline Sorbus aria (Aria edulis) & $\mathrm{E} 2 \mathrm{a}$ & . & . & . & . & + & . & . & . & 1 & 20 \\
\hline Lathyrus venetus & E1 & 2 & . & + & . & . & . & 2 & + & 2 & 40 \\
\hline Tanacetum corymbosum & E1 & 1 & + & . & . & . & . & + & . & 2 & 40 \\
\hline Arabis turrita & E1 & . & . & + & . & + & . & . & . & 2 & 40 \\
\hline Quercus pubescens & $\mathrm{E} 3 \mathrm{~b}$ & 3 & . & $\mathrm{r}$ & . & . & . & 3 & . & 2 & 40 \\
\hline Quercus pubescens & $\mathrm{E} 2 \mathrm{~b}$ & + & . & . & . & . & . & . & . & 1 & 20 \\
\hline Cnidium silaifolium & E1 & + & . & . & . & . & 1 & + & . & 1 & 20 \\
\hline Asparagus tenuifolius & E1 & + & . & . & . & . & + & . & . & 1 & 20 \\
\hline Mercurialis $x$ paxii & E1 & . & . & + & . & . & + & + & . & 1 & 20 \\
\hline Hypericum montanum & E1 & & . & . & & + & . & . & . & 1 & 20 \\
\hline Prunus mahaleb & $\mathrm{E} 2 \mathrm{~b}$ & & . & . & . & + & . & + & . & 1 & 20 \\
\hline Euonymus verrucosa & $\mathrm{E} 2 \mathrm{~b}$ & . & . & . & . & . & + & . & . & . & . \\
\hline Quercus cerris & $\mathrm{E} 3 \mathrm{~b}$ & & . & . & & . & . & 1 & 1 & & \\
\hline
\end{tabular}


Number of relevé (Zaporedna številka popisa)

Paeonia mascula agg. (P. daurica)

Aristolochia lutea

Lathyrus niger

Buglossoides purpurocaerulea

$\mathrm{QR}$ Quercetalia roboris

Serratula tinctoria

Hieracium racemosum

QF Querco-Fagetea

Hepatica nobilis

Carex digitata

Veratrum nigrum

Hedera helix

Hedera helix

Hedera helix

Lonicera xylosteum

Corylus avellana

Corylus avellana

Quercus petraea

Quercus petraea

Viola riviniana

Ulmus minor

Corylus avellana

Acer campestre

Acer campestre

Acer campestre

Acer campestre

Acer campestre

Clematis vitalba

Melica uniflora

Epipactis microphylla

Lathraea squamaria

Carex montana

VP Vaccinio-Piceetea

Solidago virgaurea

EP Erico-Pinetea

Amelanchier ovalis

Chamaecytisus hirsutus

Pinus nigra

Pinus nigra

RP Rhmano-Prunetea

Crataegus monogyna

Crataegus monogyna

Crataegus monogyna

Rosa canina

Euonymus europaea

$$
\begin{aligned}
& \begin{array}{l|lllll|lllll}
1 & 2 & 3 & 4 & 5 & 6 & 7 & 8 & \text { Pr. } & \text { Fr. }
\end{array} \\
& 10
\end{aligned}
$$

E1
E1

(1)


Number of relevé (Zaporedna številka popisa)

\begin{tabular}{l|lllll|lllll} 
& 1 & 2 & 3 & 4 & 5 & 6 & 7 & 8 & Pr. & Fr.
\end{tabular}

MuA Mulgedio-Aconitetea, Betulo-Alnetea

Saxifraga rotundifolia

TG Trifolio-Geranietea

Campanula rapunculoides

Polygonatum odoratum

Anthericum ramosum

Digitalis grandiflora

Melampyrum velebiticum

Valeriana nemorensis

Lilium carniolicum

Vincetoxicum hirundinaria

Peucedanum cervaria

Valeriana wallrothii

Silene nutans

Viola hirta

Verbascum chaixii

Thalictrum minus

Geranium sanguineum

Hypericum perforatum

Trifolium rubens

FB Festuco-Brometea

Carex humilis

Iberis linifolia

Brachypodium rupestre

Allium carinatum subsp. pulchellum

Betonica serotina

Bromopsis erecta

Dianthus tergestinus

Satureja montana subsp. variegata

Satureja subspicata subsp. liburnica

Teucrium chamaedrys

KC Koelerio-Corynephoretea

Cardaminopsis arenosa

Saxifraga tridactylites

MA Molinio-Arrhenatheretea

Muscari botryoides

Ajuga reptans

Veronica chamaedrys

Colchicum autumnale

Cy Cystopteridion fragilis

Moehringia muscosa

Pseudofumaria alba

PS Physoplexido-Saxifragion, Potentilletalia caulescentis

Campanula pyramidalis

Micromeria thymifolia

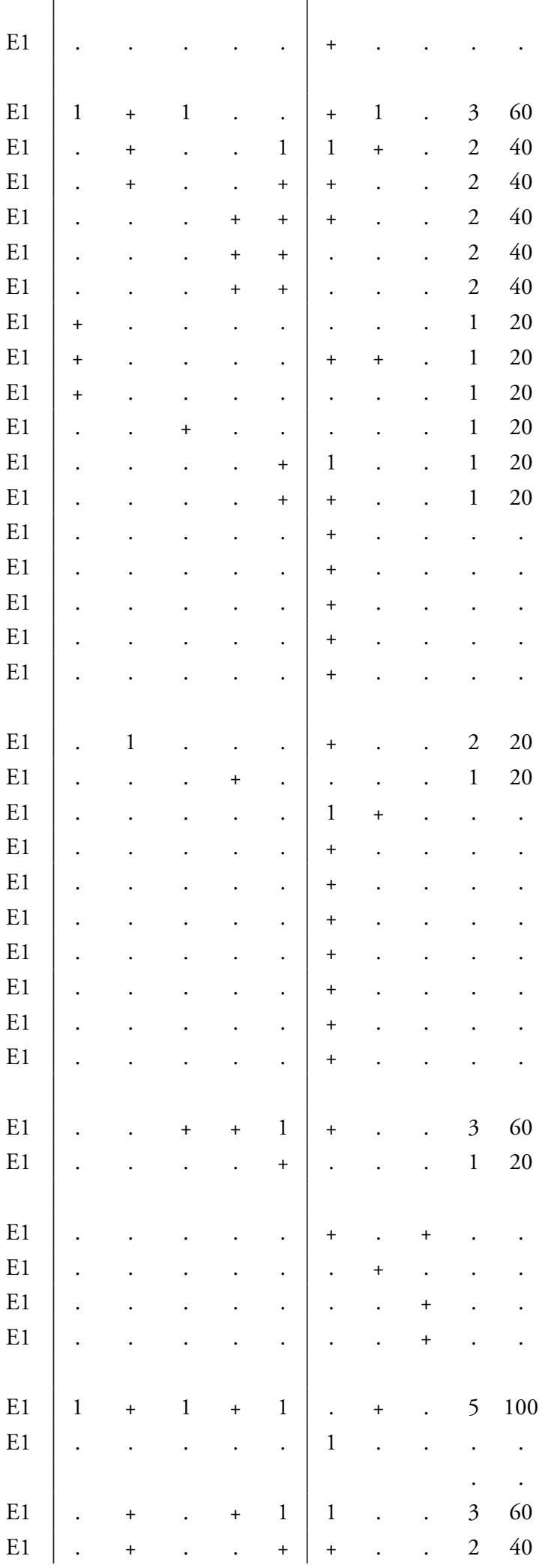




\begin{tabular}{|c|c|c|c|c|c|c|c|c|c|c|c|c|}
\hline & Number of relevé (Zaporedna številka popisa) & & 1 & 2 & 3 & 4 & 5 & 6 & 7 & 8 & Pr. & Fr. \\
\hline & Primula auricula & E1 & . & + & . & . & 1 & . & . & . & 2 & 40 \\
\hline & Hieracium lasiophyllum & E1 & . & . & . & . & + & . & . & . & 1 & 20 \\
\hline \multirow[t]{9}{*}{ AT } & Asplenietea trichomanis & & & & & & & & & & & \\
\hline & Asplenium trichomanes & E1 & + & + & . & + & 1 & 1 & + & . & 4 & 80 \\
\hline & Sedum maximum & E1 & . & . & + & 1 & 1 & 1 & . & . & 3 & 60 \\
\hline & Polypodium interjectum & E1 & . & + & . & . & 3 & . & . & . & 2 & 40 \\
\hline & Asplenium ruta-muraria & $\mathrm{E} 1$ & . & . & + & . & + & 1 & . & . & 2 & 40 \\
\hline & Polypodium vulgare & E1 & . & + & . & . & . & . & . & . & 1 & 20 \\
\hline & Polypodium australe & E1 & . & . & . & . & 1 & . & . & . & 1 & 20 \\
\hline & Parietaria judaica & E1 & . & . & . & . & + & . & . & . & 1 & 20 \\
\hline & Sedum sexangulare & E1 & . & . & . & . & . & + & . & . & . & . \\
\hline \multirow[t]{17}{*}{ ML } & Mosses and lichens (Mahovi in lišaji) & & & & & & & & & & & \\
\hline & Ctenidium molluscum & E0 & 2 & 1 & 3 & 1 & 2 & . & . & . & 5 & 100 \\
\hline & Neckera crispa & E0 & 2 & 2 & 1 & . & 2 & + & 1 & . & 4 & 80 \\
\hline & Thuidium tamariscinum & E0 & 2 & . & 2 & 3 & . & . & . & . & 3 & 60 \\
\hline & Dicranum scoparium & E0 & . & + & + & . & + & . & . & . & 3 & 60 \\
\hline & Hylocomium splendens & E0 & . & + & . & . & + & . & . & . & 2 & 40 \\
\hline & Anomodon viticulosus & E0 & . & . & + & . & 1 & 1 & 1 & 1 & 2 & 40 \\
\hline & Homalothecium lutescens & E0 & . & . & + & + & . & 2 & 1 & . & 2 & 40 \\
\hline & Peltigera canina & E0 & . & . & + & + & . & . & . & . & 2 & 40 \\
\hline & Rhytidiadelphus triquetrus & E0 & . & . & . & 2 & + & . & . & . & 2 & 40 \\
\hline & Tortella tortuosa & E0 & . & + & . & . & . & . & . & . & 1 & 20 \\
\hline & Brachythecium rutabulum & E0 & . & . & + & . & . & . & . & . & 1 & 20 \\
\hline & Neckera complanata & E0 & . & . & 1 & . & . & . & 1 & . & 1 & 20 \\
\hline & Schistidium apocarpum & E0 & . & . & + & . & . & . & . & . & 1 & 20 \\
\hline & Anomodon attenuatus & E0 & . & . & . & . & + & . & + & . & 1 & 20 \\
\hline & Thuidium delicatulum & E0 & . & . & . & . & + & . & . & . & 1 & 20 \\
\hline & Isothecium alopecuroides & E0 & . & . & . & . & . & . & . & 1 & . & 泀 \\
\hline
\end{tabular}

\section{Legend - Legenda}

1-5 Paeonio officinalis-Tilietum platyphylli hierochloetosum australis

6 Seslerio autumnalis-Ostryetum carpinifoliae

7 Seslerio autumnalis-Quercetum cerridis/Aristolochio luteae-Quercetum pubescentis carpinetosum betuli

8 Asaro-Carpinetum betuli

A Limestone - apnenec

Gr Gravel - grušč

L Marlstone - laporovec

Re Rendzina - rendzina

$\mathrm{Rj}$ Brown calcareous soil - rjava pokarbonatna tla

Pr. Presence (number of relevés in which the species is presented) - število popisov, v katerih se pojavlja vrsta

Fr. Frequency in $\%$ - frekvenca $v \%$

Helleborus multifidus subsp. istriacus* (incl. Helleborus dumetorum subsp. istriacus) - Rottensteiner (2016) 
Table 5 (Tabela 5): Lamio orvalae-Tilietum platyphylli ass. nov.

\begin{tabular}{|c|c|c|c|c|c|c|c|c|c|c|c|c|c|c|}
\hline Number of relevé (Zaporedna štev. popisa) & & 1 & 2 & 3 & 4 & 5 & 6 & 7 & 8 & 9 & 10 & 11 & 12 & 13 \\
\hline $\begin{array}{l}\text { Database number of relevé } \\
\text { (Delovna številka popisa) }\end{array}$ & & $\stackrel{\overrightarrow{\widetilde{\aleph}}}{\stackrel{\approx}{\approx}}$ & $\underset{\widetilde{N}}{\tilde{N}}$ & $\stackrel{\text { }}{\widetilde{\approx}}$ & 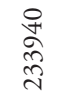 & 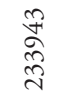 & $\underset{\widetilde{\approx}}{\stackrel{\sim}{\sim}}$ & $\underset{\widetilde{\approx}}{\stackrel{\widetilde{\vartheta}}{\sim}}$ & 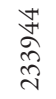 & 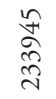 & $\underset{\substack{\sim \\
\stackrel{\sim}{\sim} \\
\stackrel{\sim}{\sim}}}{ }$ & 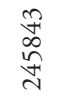 & 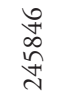 & $\underset{\substack{+\infty \\
\stackrel{\sim}{\sim} \\
\sim}}{\sim}$ \\
\hline Elevation in $\mathrm{m}$ (Nadmorska višina $\mathrm{v} \mathrm{m})$ & & 580 & 560 & 570 & 570 & 550 & 600 & 630 & 500 & 520 & 620 & 620 & 670 & 675 \\
\hline Aspect (Lega) & & NW & NW & NW & NW & NW & NNW & $\mathrm{NE}$ & $\mathrm{NE}$ & 0 & W & NW & NW & NW \\
\hline Slope in degrees (Nagib v stopinjah) & & 20 & 15 & 20 & 20 & 25 & 25 & 25 & 10 & 0 & 15 & 15 & 20 & 20 \\
\hline Parent material (Matična podlaga) & & A & A & A & A & A & A & A & A & A & DA & A & $\mathrm{D}$ & $\mathrm{D}$ \\
\hline Soil (Tla) & & $\mathrm{Rj}$ & $\mathrm{Rj}$ & $\operatorname{Re}$ & $\mathrm{Rj}$ & $\mathrm{Rj}$ & $\mathrm{Rj}$ & $\mathrm{Rj}$ & $\mathrm{Rj}$ & $\mathrm{Rj}$ & $\mathrm{Rj}$ & $\mathrm{Rj}$ & $\operatorname{Re}$ & $\operatorname{Re}$ \\
\hline Stoniness in \% (Kamnitost v \%) & & 50 & 30 & 60 & 40 & 60 & 60 & 60 & 20 & 10 & 60 & 40 & 30 & 20 \\
\hline \multicolumn{15}{|l|}{ Cover in \% (Zastiranje v \%): } \\
\hline Upper tree layer (Zgornja drevesna plast) & $\mathrm{E} 3 \mathrm{~b}$ & 90 & 90 & 80 & 90 & 80 & 80 & 80 & 70 & 70 & 80 & 70 & 80 & 70 \\
\hline Lower tree layer (Spodnja drevesna plasti) & E3a & 10 & 10 & 10 & 10 & 10 & 10 & 10 & 20 & 10 & 20 & 20 & 20 & 20 \\
\hline Shrub layer (Grmovna plast) & E2 & 10 & 20 & 20 & 20 & 20 & 30 & 30 & 40 & 20 & 20 & 30 & 20 & 30 \\
\hline Herb layer (Zeliščna plast) & E1 & 60 & 70 & 50 & 60 & 50 & 40 & 60 & 30 & 50 & 70 & 70 & 80 & 80 \\
\hline Moss layer (Mahovna plast) & E0 & 10 & 5 & 20 & 20 & 20 & 20 & 30 & 10 & 10 & 20 & 30 & 10 & 20 \\
\hline Max. tree diameter (Maks. premer dreves) & $\mathrm{cm}$ & 40 & 30 & 45 & 40 & 35 & 40 & 40 & 40 & 25 & 35 & 35 & 35 & 25 \\
\hline Max. tree height (Maks. višina dreves) & $\mathrm{m}$ & 24 & 18 & 26 & 22 & 22 & 26 & 27 & 18 & 17 & 22 & 22 & 20 & 16 \\
\hline Number of species (Število vrst) & & 51 & 51 & 55 & 53 & 62 & 50 & 57 & 48 & 42 & 85 & 88 & 76 & 58 \\
\hline Relevé area (Velikost popisne ploskve) & $\mathrm{m}^{2}$ & 400 & 400 & 400 & 400 & 400 & 400 & 400 & 200 & 400 & 200 & 400 & 400 & 200 \\
\hline Date of taking relevé (Datum popisa) & & 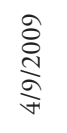 & 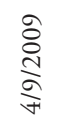 & 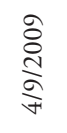 & 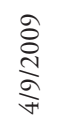 & 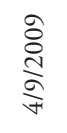 & 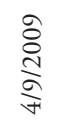 & 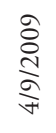 & 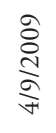 & 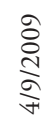 & $\frac{\stackrel{N}{\stackrel{n}{n}}}{\stackrel{m}{n}}$ & 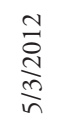 & $\frac{\stackrel{N}{a}}{\stackrel{m}{n}}$ & 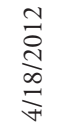 \\
\hline Locality (Nahajališče) & & 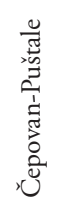 & 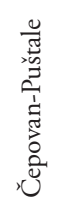 & 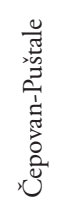 & 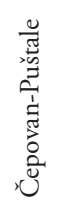 & 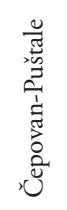 & 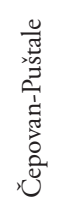 & 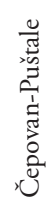 & 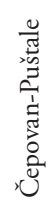 & 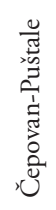 & 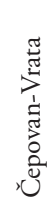 & 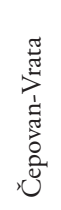 & 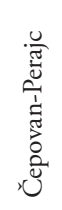 & 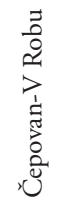 \\
\hline Quadrant (Kvadrant) & & $\begin{array}{l}\frac{+}{\infty} \\
\infty \\
\stackrel{\infty}{\sigma}\end{array}$ & $\begin{array}{l}\frac{+}{\infty} \\
\substack{\infty \\
\sigma} \\
\sigma\end{array}$ & \begin{tabular}{l}
$\frac{+}{\infty}$ \\
$\infty$ \\
\multirow{2}{+}{}
\end{tabular} & $\begin{array}{l}\frac{+1}{\infty} \\
\stackrel{\infty}{a} \\
\sigma\end{array}$ & \begin{tabular}{l}
$\frac{1}{\infty}$ \\
$\infty$ \\
\multirow{2}{*}{}
\end{tabular} & $\begin{array}{l}\frac{+}{\infty} \\
\substack{\infty \\
\sigma}\end{array}$ & 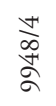 & $\begin{array}{l}\frac{+}{\infty} \\
\stackrel{\infty}{+} \\
\sigma\end{array}$ & \begin{tabular}{l}
$\frac{+}{\infty}$ \\
$\stackrel{+}{2}$ \\
\multicolumn{2}{|c}{}
\end{tabular} & $\begin{array}{l}\frac{1}{\infty} \\
\stackrel{\alpha}{\alpha}\end{array}$ & 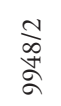 & $\begin{array}{l}\frac{N}{\infty} \\
\stackrel{+}{a}\end{array}$ & $\begin{array}{l}\frac{N}{\infty} \\
\text { के } \\
\sigma\end{array}$ \\
\hline Coordinate (Koordinate) GK Y (D-48) & $\mathrm{m}$ & $\begin{array}{l}\triangleq \\
\Xi \\
\stackrel{+}{+}\end{array}$ & $\begin{array}{l}\vec{b} \\
\stackrel{0}{\circ} \\
\stackrel{+}{+}\end{array}$ & 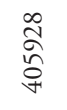 & 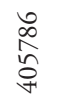 & $\begin{array}{l}\vec{N} \\
\hat{\widehat{n}} \\
\stackrel{+}{v}\end{array}$ & $\begin{array}{l}\infty \\
\stackrel{\infty}{n} \\
\hat{\sigma} \\
\hat{\sigma}\end{array}$ & 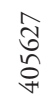 & $\begin{array}{l}\vec{\nabla} \\
\hat{n} \\
\hat{o}\end{array}$ & 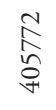 & 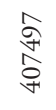 & 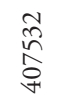 & $\begin{array}{l}\stackrel{\infty}{N} \\
\stackrel{n}{\hat{~}} \\
\text { ơ }\end{array}$ & $\stackrel{n}{n}$ \\
\hline Coordinate (Koordinate) GK X (D-48) & $\mathrm{m}$ & $\begin{array}{l}\infty \\
\stackrel{\circ}{\circ} \\
\stackrel{\circ}{\circ} \\
\stackrel{\circ}{n}\end{array}$ & $\begin{array}{l}\stackrel{\circ}{\circ} \\
\hat{\circ} \\
\hat{\circ}\end{array}$ & $\begin{array}{l}\vec{\infty} \\
\stackrel{0}{0} \\
\infty \\
\stackrel{\circ}{\circ}\end{array}$ & \begin{tabular}{l}
$\vec{\sigma}$ \\
\multirow{+}{\infty}{} \\
$\stackrel{0}{\hat{n}}$
\end{tabular} & $\begin{array}{l}m \\
\infty \\
\infty \\
\stackrel{2}{\infty} \\
\stackrel{n}{n}\end{array}$ & $\begin{array}{l}\text { तี } \\
\text { Dू } \\
\text { Oे } \\
\text { î }\end{array}$ & 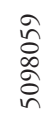 & $\begin{array}{l}\text { N } \\
\hat{n} \\
\infty \\
\hat{\infty} \\
\tilde{n}\end{array}$ & 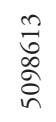 & $\begin{array}{l}\hat{\infty} \\
\approx \\
\hat{o} \\
i n\end{array}$ & \begin{tabular}{l}
$\stackrel{0}{2}$ \\
\multirow{2}{0}{} \\
0 \\
$i$ \\
$i n$
\end{tabular} & $\begin{array}{l}\frac{n}{n} \\
\hat{n} \\
\hat{o} \\
n\end{array}$ & 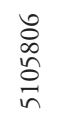 \\
\hline
\end{tabular}

Diagnostic species of the association (Diagnostične vrste asociacije)

Pr. Fr.
TA Tilia platyphyllos
TA Tilia platyphyllos
TA Tilia platyphyllos
TA Tilia platyphyllos
TA Tilia cordata
TA Tilia cordata
TA Tilia cordata
TA Tilia cordata
EC Helleborus odorus
AF Lamium orvala
AF Anemone trifolia
QP Sesleria autumnalis
TA Arum maculatum

\begin{tabular}{l|ccccccccccccc|cc} 
E3b & 2 & 4 & 3 & 4 & 3 & 4 & 3 & 2 & + & 1 & 1 & 3 & 1 & 13 & 100 \\
E3a & $\cdot$ &. &. & + & + & + & + & + &. &. &. &. &. & 5 & 38 \\
E2b & $\cdot$ &. &. & + &. & + & + &. & + &. &. &. &. & 4 & 31 \\
E1 &. &. &. &. &. &. &. &. &. &. &. &. & + & 1 & 8 \\
E3b & 2 & + & 2 & 2 & 2 & + & 1 & 3 & 4 & 3 & 4 & 3 & 3 & 13 & 100 \\
E3a & 1 & + & + & + & + & + &. & 1 &. & 1 &. &. & 1 & 9 & 69 \\
E2b &. &. & + &. &. &. &. & 1 &. &. & + &. &. & 3 & 23 \\
E2a &. &. &. &. &. &. &. &. &. & + & + &. & + & 3 & 23 \\
E1 & 2 & 2 & + & 1 & 1 &. &. & 1 & 1 & + & 2 & 2 & 2 & 11 & 85 \\
E1 & 1 & 1 & 1 & 1 & 1 & + & 1 &. &. & 1 & 1 & + & 1 & 11 & 85 \\
E1 & 1 &. & 1 & 1 & 1 & 1 & 1 & + &. & + & 1 & 1 &. & 10 & 77 \\
E1 & 2 & 3 &. & 1 & 1 & 1 & + & + & + &. &. &. &. & 62 \\
E1 &. &. & 1 & 1 & 1 & + & 1 & + & + &. &. &. & + & 6 \\
8 & 62
\end{tabular}




\section{Number of relevé (Zaporedna štev. popisa)}

Differential species of variants (Razlikovalnica variante)

TA Adoxa moschatellina

AF Omphalodes verna

TA Tilio-Acerion

Corydalis solida

Acer pseudoplatanus

Acer pseudoplatanus

Acer pseudoplatanus

Acer pseudoplatanus

Acer pseudoplatanus

Polystichum aculeatum

Phyllitis scolopendrium

E

Acer platanoides

Acer platanoides

Acer platanoides

Aruncus dioicus

Isopyrum thalictroides

Geranium robertianum

Euonymus latifolia

Euonymus latifolia

Lunaria rediviva

Stellaria montana

Juglans regia

Ulmus glabra

EC Erythronio-Carpinion

Galanthus nivalis

Primula vulgaris

Crocus vernus subsp. vernus*

Ornithogalum pyrenaicum

AF Aremonio-Fagion

Cyclamen purpurascens

Cardamine enneaphyllos

Cardamine trifolia

Euphorbia carniolica

Anemone x pittonii

Daphne laureola

Rhamnus fallax

Aremonia agrimonoides

Helleborus niger

AI Alnion incanae

Chrysosplenium alternifolium

Dryopteris carthusiana

FS Fagetalia sylvaticae

Asarum europaeum subsp. caucasicum

Mercurialis perennis

Paris quadrifolia

Daphne mezereum

Fraxinus excelsior

Fraxinus excelsior

Fraxinus excelsior

E1

E3b

E3a

E2b

E2a

E1

E1

E1

E3b

E3a

E1

E1

E1

E1

E2a

E1

E1

E1

E3b

E2a

E1

E1

E1

E1

E1

E1

E1

E1

E1

E2a

E2a

E1

E

E1

E1

E1

E1

E1

E2a

E3b

E3a

E2b

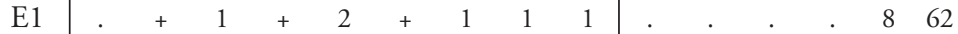

. . . . . . . . $4+++4431$

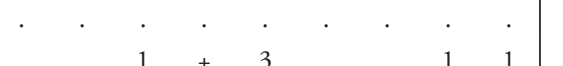

$1+3 \cdot 1+1+4.954$

. . ..+++1.646

. . . . . . $+4+.431$

. . . . . $+.+2+215$

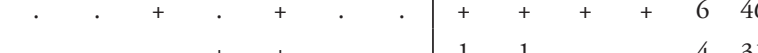

..+

. . . . . . + + + +323

. . . . . . ++423

. . . . . . + . . . 215

. . . . . . + + +215

. . . . . . . + + 2

. . . . 1 . 1 .

. . . . + . . . . . . .

a

$+18$

$7 \quad 54$

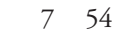

$\begin{array}{ll}4 & 31\end{array}$

$\begin{array}{ll}1 & 8\end{array}$

754

$+754$

538

$\begin{array}{ll}3 & 23\end{array}$

215

215

215

2 15

18

215

18

100

3100

92

85

85

15

23 


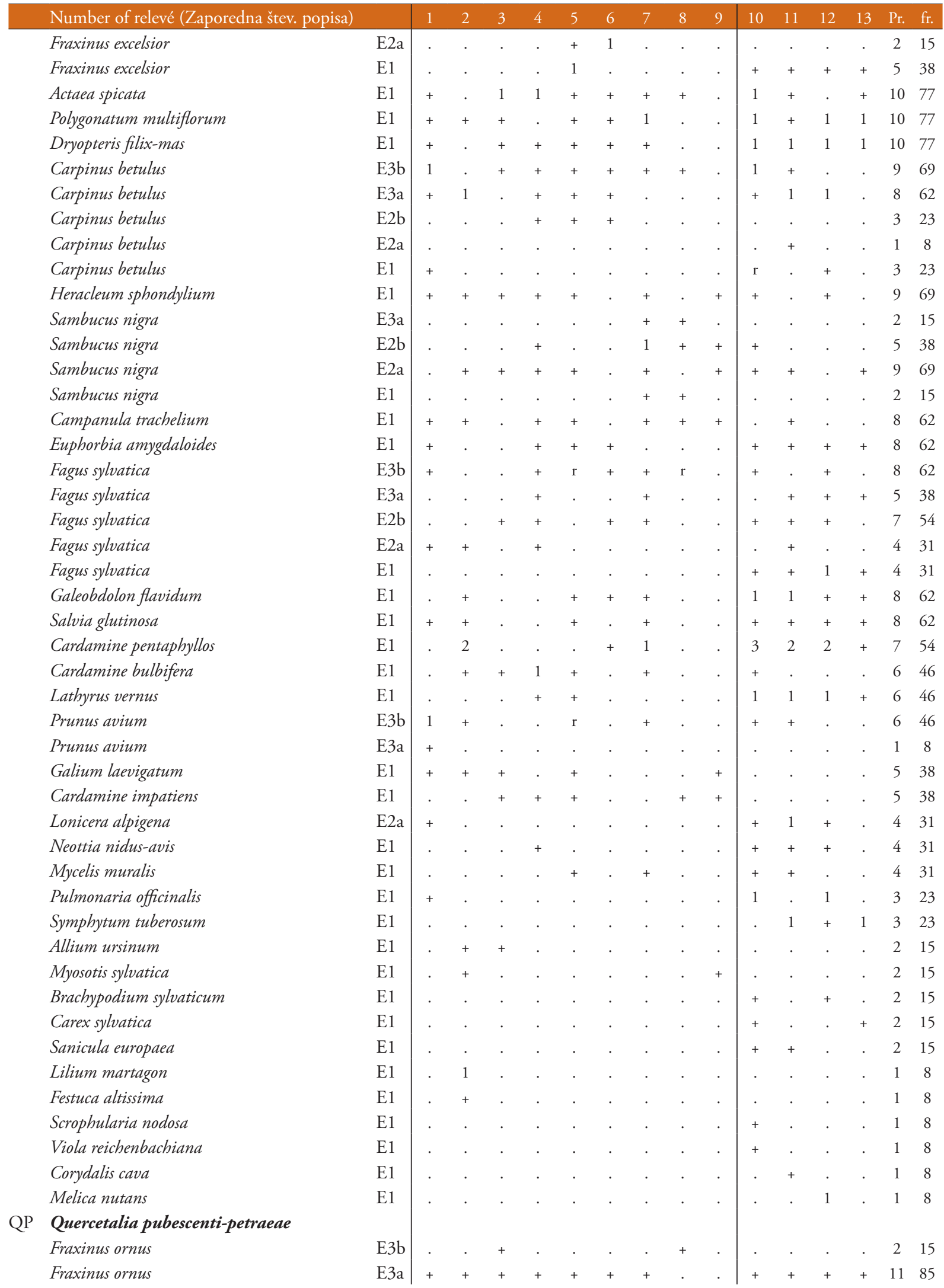




\begin{tabular}{|c|c|c|c|c|c|c|c|c|c|c|c|c|c|c|c|c|c|}
\hline & \multicolumn{2}{|l|}{ Number of relevé (Zaporedna štev. popisa) } & 1 & 2 & 3 & 4 & 5 & 6 & 7 & 8 & 9 & 10 & 11 & 12 & 13 & Pr. & fr. \\
\hline & Fraxinus ornus & $\mathrm{E} 2 \mathrm{~b}$ & . & . & . & . & . & . & . & . & . & . & + & . & . & 1 & 8 \\
\hline & Fraxinus ornus & E2a & . & . & . & + & . & . & . & . & . & . & . & + & . & 2 & 15 \\
\hline & Ostrya carpinifolia & $\mathrm{E} 3 \mathrm{~b}$ & . & . & + & + & + & + & + & + & . & + & + & 1 & + & 10 & 77 \\
\hline & Ostrya carpinifolia & E3a & . & . & + & . & . & . & + & . & . & + & . & . & . & 3 & 23 \\
\hline & Cornus mas & $\mathrm{E} 2 \mathrm{~b}$ & . & . & . & . & r & . & $\mathrm{r}$ & . & . & . & . & . & . & 2 & 15 \\
\hline & Orchis mascula subsp. speciosa & E1 & . & + & . & . & . & . & . & . & . & . & . & + & . & 2 & 15 \\
\hline & Tanacetum corymbosum & E1 & + & . & . & . & . & . & . & . & . & . & . & . & . & 1 & 8 \\
\hline & Sorbus aria (Aria edulis) & E3a & . & . & . & . & . & . & $\mathrm{r}$ & . & . & . & . & . & . & 1 & 8 \\
\hline \multirow[t]{29}{*}{ QF } & \multicolumn{17}{|l|}{ Querco-Fagetea } \\
\hline & Corylus avellana & E3a & + & 1 & 1 & 1 & . & . & + & 2 & 1 & . & 2 & . & . & 8 & 62 \\
\hline & Corylus avellana & $\mathrm{E} 2 \mathrm{~b}$ & 1 & 1 & 1 & 1 & 1 & 2 & 1 & 1 & 2 & 1 & 1 & 1 & 2 & 13 & 100 \\
\hline & Anemone nemorosa & $\mathrm{E} 1$ & 1 & 1 & + & 1 & . & + & . & 1 & + & 1 & 2 & 2 & 3 & 11 & 85 \\
\hline & Lonicera xylosteum & $\mathrm{E} 2 \mathrm{~b}$ & . & . & . & . & . & + & + & . & . & . & . & . & . & 2 & 15 \\
\hline & Lonicera xylosteum & E2a & + & + & 1 & + & + & + & + & . & . & + & 1 & + & + & 11 & 85 \\
\hline & Carex digitata & E1 & + & + & + & + & . & + & . & . & . & 1 & . & + & + & 8 & 62 \\
\hline & Aegopodium podagraria & E1 & + & 1 & + & . & . & . & . & + & 1 & . & . & + & 1 & 7 & 54 \\
\hline & Anemone ranunculoides & E1 & + & . & + & + & . & . & . & . & 1 & + & 1 & . & . & 6 & 46 \\
\hline & Acer campestre & $\mathrm{E} 3 \mathrm{~b}$ & + & . & . & + & + & . & . & + & + & . & . & . & . & 5 & 38 \\
\hline & Acer campestre & E3a & + & + & . & + & . & . & . & . & . & . & . & . & . & 3 & 23 \\
\hline & Acer campestre & E1 & . & . & . & . & . & . & . & . & . & . & . & . & + & 1 & 8 \\
\hline & Dactylis glomerata subsp. lobata (D. polygama) & E1 & + & 1 & + & + & + & . & . & . & . & . & . & . & . & 5 & 38 \\
\hline & Festuca heterophylla & E1 & + & + & . & . & . & . & . & . & . & + & + & + & . & 5 & 38 \\
\hline & Gagea lutea & E1 & + & . & + & . & 1 & . & r. & 1 & + & . & . & . & . & 5 & 38 \\
\hline & Listera ovata & E1 & + & . & . & . & . & . & . & . & . & + & + & + & . & 4 & 31 \\
\hline & Hedera helix & E3a & . & . & . & . & . & . & . & . & . & . & + & . & + & 2 & 15 \\
\hline & Hedera helix & E1 & . & . & . & . & . & . & . & . & . & + & + & + & 1 & 4 & 31 \\
\hline & Dactylorhiza fuchsii & E1 & . & . & . & . & . & . & . & . & . & + & + & + & . & 3 & 23 \\
\hline & Lathraea squamaria & E1 & + & + & . & . & . & . & . & . & . & . & . & . & . & 2 & 15 \\
\hline & Veratrum nigrum & E1 & . & + & . & . & . & . & . & . & . & $\mathrm{r}$ & . & . & . & 2 & 15 \\
\hline & Vinca minor & E1 & . & . & + & . & $\cdot$ & . & . & . & . & . & . & 2 & . & 2 & 15 \\
\hline & Moehringia trinervia & E1 & . & . & $\cdot$ & . & . & . & . & + & + & . & . & . & . & 2 & 15 \\
\hline & Hepatica nobilis & E1 & . & . & . & . & $\cdot$ & . & . & . & . & + & + & . & . & 2 & 15 \\
\hline & Clematis vitalba & E1 & . & . & . & . & · & . & . & . & . & + & . & . & + & 2 & 15 \\
\hline & Cruciata glabra & E1 & . & . & . & . & . & . & . & . & . & + & . & . & + & 2 & 15 \\
\hline & Viola riviniana & E1 & . & . & . & . & · & . & . & . & . & . & + & . & + & 2 & 15 \\
\hline & Malus sylvestris & E1 & . & . & . & . & . & . & . & . & . & . & + & . & . & 1 & 8 \\
\hline & Pyrus pyraster & E2a & . & . & $\cdot$ & . & $\cdot$ & . & . & . & . & . & $\mathrm{r}$ & . & . & 1 & 8 \\
\hline \multirow[t]{13}{*}{ VP } & \multicolumn{17}{|l|}{ Vaccinio-Piceetea } \\
\hline & Oxalis acetosella & E1 & . & . & 1 & 1 & + & + & . & + & . & 1 & 1 & + & 1 & 9 & 69 \\
\hline & Luzula luzuloides & E1 & . & . & . & . & + & + & + & + & . & + & + & + & . & 7 & 54 \\
\hline & Solidago virgaurea & E1 & . & . & . & . & . & . & . & . & + & . & + & + & . & 3 & 23 \\
\hline & Picea abies & E3b & . & . & $\mathrm{r}$ & . & . & . & . & . & . & + & + & + & . & 4 & 31 \\
\hline & Picea abies & E3a & . & . & . & . & . & $\mathrm{r}$ & . & . & . & . & . & . & . & 1 & 8 \\
\hline & Picea abies & E2b & . & . & . & . & . & . & . & . & . & + & . & + & + & 3 & 23 \\
\hline & Picea abies & E1 & . & . & . & . & . & . & . & . & . & + & . & . & . & 1 & 8 \\
\hline & Maianthemum bifolium & E1 & . & . & . & . & . & . & . & . & . & + & + & . & . & 2 & 15 \\
\hline & Picea abies & $\mathrm{E} 2 \mathrm{a}$ & . & . & . & . & . & . & . & . & . & . & + & . & + & 2 & 15 \\
\hline & Larix decidua & E3b & . & . & $\mathrm{r}$ & . & . & . & . & . & . & . & . & . & . & 1 & 8 \\
\hline & Dryopteris dilatata & E1 & . & . & $\cdot$ & . & + & . & . & . & . & . & . & . & . & 1 & 8 \\
\hline & Gentiana asclepiadea & E1 & . & . & . & . & . & . & . & . & . & . & + & . & . & 1 & 8 \\
\hline
\end{tabular}


\begin{tabular}{l|llllllllll|lllllll} 
Number of relevé (Zaporedna štev. popisa) & 1 & 2 & 3 & 4 & 5 & 6 & 7 & 8 & 9 & 10 & 11 & 12 & 13 & Pr. & fr.
\end{tabular}

EP Erico-Pinetea

Calamagrostis varia

Peucedanum austriacum

E1

Pinus nigra

Rubus saxatilis

Aquilegia nigricans

Carex alba

SSC Sambuco-Salicion capreae

Salix caprea

Salix caprea

Betula pendula

Sorbus aucuparia

$\mathrm{RP}$

Rhamno-Prunetea

Crataegus monogyna

Crataegus monogyna

Crataegus monogyna

Euonymus europaea

Rubus hirtus

Berberis vulgaris

MuA Mulgedio-Aconitetea

Senecio ovatus

Aconitum lycoctonum

Athyrium filix-femina

Milium effusum

Polygonatum verticillatum

EA Epilobietea angustifolii

Fragaria vesca

GU Galio-Urticetea

Geum urbanum

Urtica dioica

Alliaria petiolata

Allium vineale

TG Trifolio-Geranietea

Campanula rapunculoides

Lilium bulbiferum

Valeriana wallrothii

FB Festuco-Brometea

Allium carinatum subsp. carinatum

Cirsium erisithales

Euphorbia cyparissias

MA Molinio-Arrbenatheretea

Angelica sylvestris

Veronica chamaedrys

Colchicum autumnale

Anthriscus sylvestris

Crocus albiflorus

Poa trivialis

Galium mollugo

Asplenietea trichomanis s. lat.

Asplenium trichomanes
E1

E3b

E1

E1

E1

$\mathrm{E} 3 \mathrm{~b}$

E3a

$\mathrm{E} 3 \mathrm{~b}$

$\mathrm{E} 3 \mathrm{~b}$

E2a

E2

E1

E1

E2a

E2a

E1

E1

E1

E1

E

E1

E1

E1

E1

E1

E1

E1

E1

E1

E1

E1

E1

E1

E1

E1

E1

E1

E1

E1$$
\text { E1 }
$$

$$
\begin{aligned}
& +++\quad+431 \\
& \mathrm{r}++.323 \\
& \text {. . . } 18 \\
& \text {. . . } 18 \\
& +. \quad 18 \\
& +\quad 18 \\
& \text {. } 18 \\
& \text {. } 215 \\
& \text {. } \quad 18 \\
& 18 \\
& 215 \\
& 215 \\
& \begin{array}{ll}
1 & 8
\end{array} \\
& \begin{array}{ll}
1 & 8
\end{array} \\
& 18 \\
& \begin{array}{ll}
1 & 8
\end{array} \\
& 754 \\
& 215 \\
& 215 \\
& \begin{array}{ll}
1 & 8
\end{array} \\
& \begin{array}{ll}
1 & 8
\end{array} \\
& 646 \\
& \begin{array}{ll}
8 \quad 62 \\
2
\end{array} \\
& \begin{array}{ll}
4 & 31
\end{array} \\
& 18 \\
& +18 \\
& +323 \\
& \text { r. } 18 \\
& +18 \\
& \text {. } 323 \\
& +\quad 323 \\
& \text {. } 18 \\
& +646 \\
& \text {. } 323 \\
& \text {. } 18 \\
& \text {. } 18 \\
& \text {. } 18 \\
& \text {. } 18 \\
& +18
\end{aligned}
$$




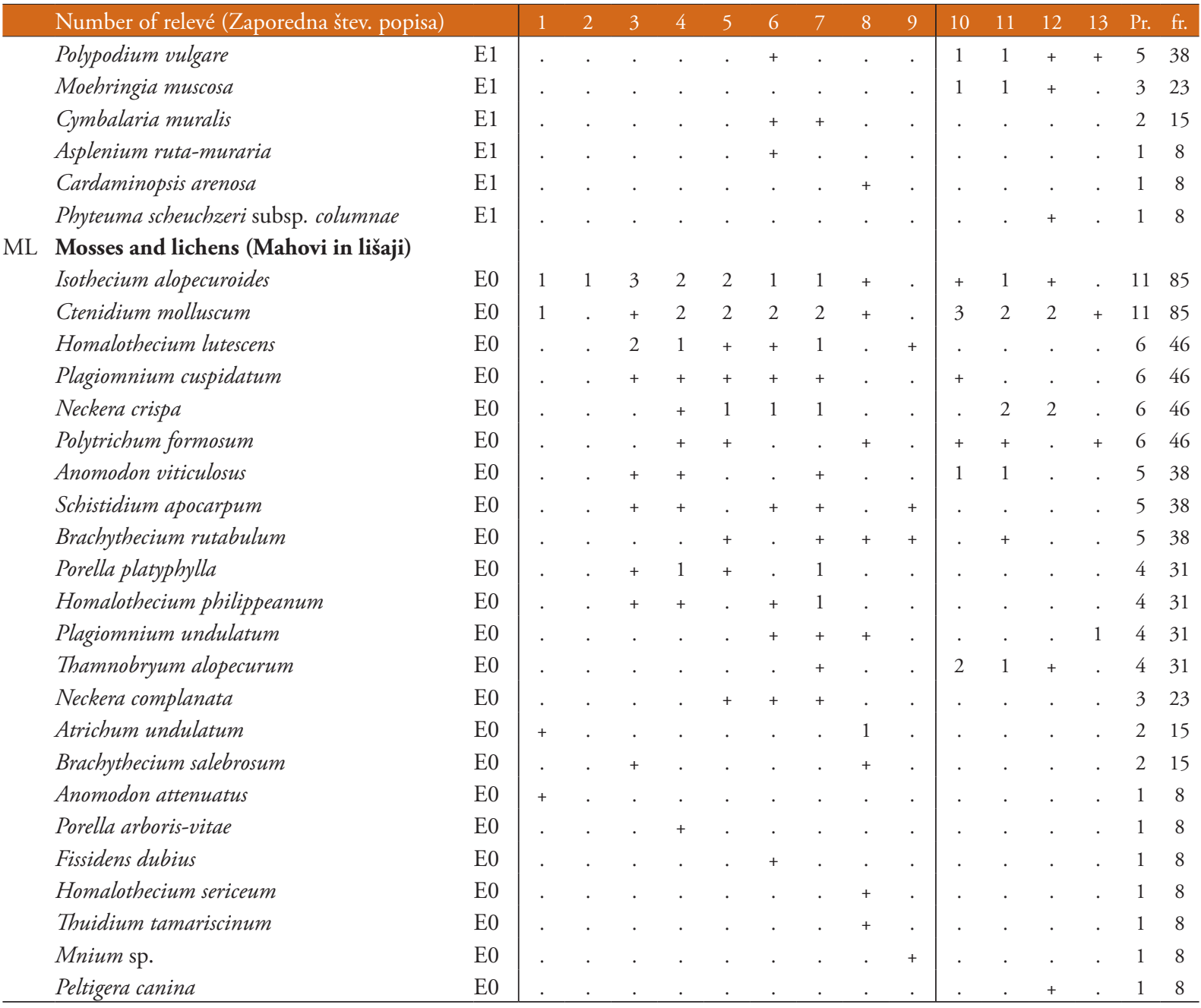

\section{Legend - Legenda}
A Limestone - apnenec
D Dolomite - dolomit
Re Rendzina - rendzina
$\mathrm{Rj}$ Brown calcareous soil - rjava pokarbonatna tla
Pr. Presence (number of relevés in which the species is presented) - število popisov, v katerih se pojavlja vrsta
Fr. Frequency in $\%-$ frekvenca $v \%$

Crocus vernus subsp. vernus* is according to new findings Crocus heuffellianus Herb. (Peruzzi 2016) 
Table 6: Synoptic table of syntaxa from the suballiance Ostryo-Tilienion platyphylli in the (south)western Slovenia.

Tabela 6: Sintezna tabela sintaxonov podzveze Ostryo-Tilienion platyphylli v (jugo)zahodni Sloveniji.

\begin{tabular}{|c|c|c|c|c|c|c|c|}
\hline Successive number (Zaporedna številka) & & 1 & 2 & 3 & 4 & 5 & 6 \\
\hline Sign for syntaxa (Oznaka sintaksona) & & CoAp & RaAp & PoTpsr & PoTpcs & PoTpha & LoTp \\
\hline Number of relevés (Število popisov) & & 10 & 13 & 8 & 5 & 5 & 13 \\
\hline \multicolumn{8}{|l|}{ Tilio-Acerion } \\
\hline Adoxa moschatellina & E1 & 90 & . & 88 & . & 20 & 62 \\
\hline Acer pseudoplatanus & E3b & 90 & 100 & 38 & 60 & 60 & 46 \\
\hline Acer pseudoplatanus & E3a & 10 & 38 & 13 & 20 & . & 31 \\
\hline Acer pseudoplatanus & $\mathrm{E} 2 \mathrm{~b}$ & 10 & 38 & 38 & 20 & 20 & 15 \\
\hline Acer pseudoplatanus & $\mathrm{E} 2 \mathrm{a}$ & 20 & 46 & 50 & 40 & 20 & 8 \\
\hline Acer pseudoplatanus & E1 & 70 & 92 & 88 & 80 & 40 & 38 \\
\hline Tilia platyphyllos & $\mathrm{E} 3 \mathrm{~b}$ & 90 & 15 & 100 & 100 & 80 & 100 \\
\hline Tilia platyphyllos & E3a & 20 & 15 & 13 & 80 & 100 & 38 \\
\hline Tilia platyphyllos & $\mathrm{E} 2 \mathrm{~b}$ & 10 & . & 13 & 60 & 40 & 31 \\
\hline Tilia platyphyllos & E2a & 10 & . & 38 & 20 & 40 & 8 \\
\hline Tilia platyphyllos & $\mathrm{E} 1$ & . & . & 75 & 20 & . & . \\
\hline Tilia cordata & E3b & 90 & 8 & 13 & 60 & 40 & 100 \\
\hline Tilia cordata & E3a & 30 & . & . & 20 & 60 & 69 \\
\hline Tilia cordata & $\mathrm{E} 2 \mathrm{~b}$ & . & . & . & 20 & . & 23 \\
\hline Tilia cordata & $\mathrm{E} 2 \mathrm{a}$ & 10 & . & . & 20 & 20 & 23 \\
\hline Isopyrum thalictroides & $\mathrm{E} 1$ & 70 & 62 & . & . & 40 & 23 \\
\hline Geranium robertianum & E1 & 60 & 38 & 63 & 20 & 60 & 15 \\
\hline Aruncus dioicus & E1 & 60 & 15 & . & 20 & 60 & 23 \\
\hline Corydalis solida & E1 & 50 & . & 63 & . & . & 54 \\
\hline Ulmus glabra & E3b & 20 & 54 & 13 & 100 & . & . \\
\hline Ulmus glabra & E3a & 60 & 54 & . & 60 & 20 & . \\
\hline Ulmus glabra & $\mathrm{E} 2 \mathrm{~b}$ & . & 92 & . & 80 & . & . \\
\hline Ulmus glabra & $\mathrm{E} 2 \mathrm{a}$ & 50 & 62 & 13 & 60 & 20 & 8 \\
\hline Ulmus glabra & E1 & 30 & 31 & 13 & 80 & . & . \\
\hline Phyllitis scolopendrium & E1 & 40 & 85 & . & . & 20 & 31 \\
\hline Thalictrum aquilegiifolium & E1 & 30 & . & 50 & . & . & . \\
\hline Acer platanoides & E3b & 20 & . & . & 20 & 20 & 8 \\
\hline Acer platanoides & E3a & 10 & . & . & . & . & 8 \\
\hline Acer platanoides & $\mathrm{E} 2 \mathrm{~b}$ & . & . & . & . & . & . \\
\hline Acer platanoides & $\mathrm{E} 2 \mathrm{a}$ & 20 & . & . & . & 20 & . \\
\hline Acer platanoides & E1 & 20 & . & . & 20 & 20 & 23 \\
\hline Juglans regia & E3b & . & 15 & . & . & . & 8 \\
\hline Juglans regia & $\mathrm{E} 2 \mathrm{a}$ & 10 & 15 & . & . & . & . \\
\hline Polystichum setiferum & E1 & . & 85 & . & . & . & . \\
\hline Lunaria rediviva & E1 & . & 69 & . & . & . & 8 \\
\hline Tephroseris pseudocrispa & E1 & . & 8 & . & . & . & . \\
\hline Dryopteris affinis & E1 & . & 8 & . & . & . & . \\
\hline Polystichum aculeatum & E1 & . & 8 & . & . & . & . \\
\hline Glechoma hirsuta & $\mathrm{E} 1$ & . & . & 13 & . & . & . \\
\hline Arum maculatum & $\mathrm{E} 1$ & . & . & . & . & . & 62 \\
\hline Euonymus latifolia & E2a & . & . & . & . & . & 15 \\
\hline Euonymus latifolia & E1 & . & . & . & . & . & 15 \\
\hline Stellaria montana & E1 & . & . & . & . & . & 8 \\
\hline
\end{tabular}




\begin{tabular}{|c|c|c|c|c|c|c|c|}
\hline Successive number (Zaporedna številka) & & 1 & 2 & 3 & 4 & 5 & 6 \\
\hline \multicolumn{8}{|l|}{ Erythronio-Carpinion } \\
\hline Galanthus nivalis & E1 & 100 & 85 & 100 & 60 & 100 & 54 \\
\hline Primula vulgaris & E1 & 60 & 8 & 63 & 40 & 80 & 54 \\
\hline Helleborus odorus & E1 & . & 69 & . & 20 & . & 85 \\
\hline Crocus vernus subsp. vernus* & E1 & . & 38 & 13 & . & . & . \\
\hline Erythronium dens-canis & E1 & . & . & 63 & . & . & 31 \\
\hline Ornithogalum pyrenaicum & E1 & . & . & 38 & . & . & 8 \\
\hline Lonicera caprifolium & E2a & . & . & . & 40 & . & . \\
\hline \multicolumn{8}{|l|}{ Aremonio-Fagion } \\
\hline Lamium orvala & E1 & 100 & 100 & 88 & 60 & . & 85 \\
\hline Cardamine enneaphyllos & E1 & 90 & 77 & 38 & 100 & 60 & 54 \\
\hline Cyclamen purpurascens & E1 & 70 & 38 & 88 & 100 & 100 & 54 \\
\hline Hacquetia epipactis & E1 & 30 & 54 & . & 60 & . & . \\
\hline Geranium nodosum & E1 & . & . & 88 & . & . & . \\
\hline Calamintha grandiflora & E1 & . & . & 63 & . & . & . \\
\hline Anemone trifolia & E1 & . & . & . & . & . & 71 \\
\hline Cardamine trifolia & E1 & . & . & . & . & . & 38 \\
\hline Omphalodes verna & E1 & . & . & . & . & . & 31 \\
\hline Euphorbia carniolica & E1 & . & . & . & . & . & 23 \\
\hline Anemone $x$ pittonii & $\mathrm{E} 1$ & . & . & . & . & . & 15 \\
\hline Daphne laureola & $\mathrm{E} 2 \mathrm{a}$ & . & . & . & . & . & 15 \\
\hline Rhamnus fallax & $\mathrm{E} 2 \mathrm{a}$ & . & . & . & . & . & 15 \\
\hline Aremonia agrimonoides & E1 & . & . & . & . & . & 15 \\
\hline Helleborus niger & E1 & . & . & . & . & . & 8 \\
\hline \multicolumn{8}{|l|}{ Alnion incanae } \\
\hline Aesculus hippocastanum & $\mathrm{E} 3 \mathrm{~b}$ & . & . & 13 & . & . & . \\
\hline Aesculus hippocastanum & E3a & 10 & . & . & . & . & . \\
\hline Aesculus hippocastanum & $\mathrm{E} 2 \mathrm{a}$ & . & . & . & . & 20 & . \\
\hline Rubus caesius & E1 & . & . & 13 & 20 & . & . \\
\hline Alnus glutinosa & $\mathrm{E} 3 \mathrm{~b}$ & . & 8 & . & . & . & . \\
\hline Festuca gigantea & E1 & . & 8 & . & . & . & . \\
\hline Chrysosplenium alternifolium & E1 & . & . & . & . & . & 15 \\
\hline Dryopteris carthusiana & E1 & . & . & . & . & . & 8 \\
\hline \multicolumn{8}{|l|}{ Fagetalia sylvaticae } \\
\hline Asarum europaeum subsp. caucasicum & E1 & 100 & 92 & 100 & 80 & 60 & 100 \\
\hline Polygonatum multiflorum & E1 & 100 & 85 & 63 & 60 & 80 & 77 \\
\hline Carpinus betulus & $\mathrm{E} 3 \mathrm{~b}$ & 100 & 46 & 25 & . & 40 & 69 \\
\hline Carpinus betulus & E3a & 50 & 15 & 38 & . & 40 & 62 \\
\hline Carpinus betulus & $\mathrm{E} 2 \mathrm{~b}$ & 20 & . & 13 & . & 20 & 23 \\
\hline Carpinus betulus & E2a & . & 15 & . & . & . & 8 \\
\hline Carpinus betulus & E1 & . & 15 & . & . & . & 23 \\
\hline Mercurialis perennis & E1 & 90 & 100 & 88 & 100 & 20 & 100 \\
\hline Lathyrus vernus & E1 & 90 & 31 & 75 & 100 & 80 & 46 \\
\hline Corydalis cava & E1 & 80 & 85 & 25 & . & . & 8 \\
\hline Galeobdolon flavidum & E1 & 80 & 46 & . & 80 & 40 & 62 \\
\hline Actaea spicata & E1 & 80 & 31 & 63 & . & . & 77 \\
\hline Mycelis muralis & E1 & 70 & 54 & 88 & 80 & 60 & 31 \\
\hline Galium laevigatum & E1 & 70 & . & . & 40 & 100 & 38 \\
\hline Viola reichenbachiana & E1 & 60 & 46 & . & . & . & 8 \\
\hline
\end{tabular}




\begin{tabular}{|c|c|c|c|c|c|c|c|}
\hline Successive number (Zaporedna številka) & & 1 & 2 & 3 & 4 & 5 & 6 \\
\hline Heracleum sphondylium & E1 & 50 & . & 100 & . & . & 69 \\
\hline Dryopteris filix-mas & E1 & 50 & 31 & 88 & 60 & . & 77 \\
\hline Campanula trachelium & E1 & 50 & . & 63 & 80 & 60 & 62 \\
\hline Salvia glutinosa & E1 & 50 & 69 & 100 & 80 & . & 62 \\
\hline Sambucus nigra & E3 & . & . & . & . & . & 15 \\
\hline Sambucus nigra & $\mathrm{E} 2 \mathrm{~b}$ & 20 & 85 & 13 & 40 & . & 38 \\
\hline Sambucus nigra & $\mathrm{E} 2 \mathrm{a}$ & 50 & 38 & 25 & . & . & 69 \\
\hline Sanicula europaea & E1 & 50 & . & . & . & . & 15 \\
\hline Symphytum tuberosum & E1 & 40 & 62 & 63 & 60 & . & 23 \\
\hline Fraxinus excelsior & $\mathrm{E} 3 \mathrm{~b}$ & 10 & . & 50 & . & . & 85 \\
\hline Fraxinus excelsior & E2a & 30 & . & . & 40 & . & 23 \\
\hline Fraxinus excelsior & E1 & . & . & 38 & . & . & 38 \\
\hline Cardamine bulbifera & E1 & 20 & 85 & . & 20 & . & 46 \\
\hline Euphorbia dulcis & E1 & 20 & 23 & 63 & 40 & . & . \\
\hline Paris quadrifolia & E1 & 20 & . & 25 & . & . & 92 \\
\hline Melica nutans & $\mathrm{E} 1$ & 20 & . & 25 & . & . & . \\
\hline Neottia nidus-avis & E1 & 20 & . & . & . & . & 31 \\
\hline Pulmonaria officinalis & E1 & 10 & 54 & 75 & 20 & . & 23 \\
\hline Galeobdolon montanum & E1 & 10 & 31 & 63 & . & 20 & . \\
\hline Cardamine impatiens & E1 & 10 & 38 & . & . & . & 38 \\
\hline Circaea lutetiana & E1 & 10 & 23 & . & . & . & . \\
\hline Epipactis helleborine & E1 & 10 & . & . & . & 20 & . \\
\hline Lonicera alpigena & $\mathrm{E} 2 \mathrm{a}$ & 10 & . & . & . & . & 31 \\
\hline Lilium martagon & E1 & . & 54 & . & 60 & . & 8 \\
\hline Brachypodium sylvaticum & E1 & . & 31 & . & . & . & 15 \\
\hline Fagus sylvatica & $\mathrm{E} 3 \mathrm{~b}$ & . & . & 13 & 60 & . & 62 \\
\hline Fagus sylvatica & E3a & . & 8 & . & 40 & . & 38 \\
\hline Fagus sylvatica & $\mathrm{E} 2 \mathrm{~b}$ & . & 15 & . & . & . & 54 \\
\hline Fagus sylvatica & $\mathrm{E} 2 \mathrm{a}$ & . & 8 & . & . & . & 31 \\
\hline Fagus sylvatica & E1 & . & 8 & 13 & . & . & 31 \\
\hline Prunus avium & $\mathrm{E} 3 \mathrm{~b}$ & . & 8 & 25 & . & . & 46 \\
\hline Prunus avium & E1 & . & . & 13 & . & . & . \\
\hline Carex sylvatica & $\mathrm{E} 1$ & . & 8 & . & . & . & 15 \\
\hline Scrophularia nodosa & E1 & . & 8 & 38 & . & . & 8 \\
\hline Prenanthes purpurea & E1 & . & 8 & . & 20 & . & . \\
\hline Ranunculus lanuginosus & E1 & . & . & 25 & . & . & . \\
\hline Laburnum alpinum & E3a & . & . & . & 20 & . & . \\
\hline Laburnum alpinum & $\mathrm{E} 2 \mathrm{~b}$ & . & . & . & 20 & . & . \\
\hline Laburnum alpinum & $\mathrm{E} 2 \mathrm{a}$ & . & . & . & 20 & . & . \\
\hline Laburnum alpinum & E1 & . & . & . & 20 & . & . \\
\hline Daphne mezereum & E2a & . & . & . & . & . & 85 \\
\hline Euphorbia amygdaloides & E1 & . & . & . & . & . & 62 \\
\hline Cardamine pentaphyllos & E1 & . & . & . & . & . & 54 \\
\hline Myostis sylvatica agg. & $\mathrm{E} 1$ & . & . & . & . & . & 15 \\
\hline Allium ursinum & E1 & . & . & . & . & . & 15 \\
\hline Festuca altissima & E1 & . & . & . & . & . & 8 \\
\hline \multicolumn{8}{|l|}{ Carpinion orientalis } \\
\hline Sesleria autumnalis & E1 & 80 & 8 & 100 & 100 & 100 & 62 \\
\hline Carpinus orientalis & E3a & 20 & . & . & . & . & . \\
\hline
\end{tabular}




\begin{tabular}{|c|c|c|c|c|c|c|c|}
\hline Successive number (Zaporedna številka) & & 1 & 2 & 3 & 4 & 5 & 6 \\
\hline Ruscus aculeatus & $\mathrm{E} 2 \mathrm{a}$ & . & 92 & . & 80 & . & . \\
\hline Asparagus acutifolius & $\mathrm{E} 2 \mathrm{a}$ & . & 31 & . & . & . & . \\
\hline Acer monspessulanum & $\mathrm{E} 3 \mathrm{~b}$ & . & . & . & 20 & . & . \\
\hline Acer monspessulanum & $\mathrm{E} 2 \mathrm{a}$ & . & . & . & . & 40 & . \\
\hline Frangula rupestris & $\mathrm{E} 2 \mathrm{a}$ & . & . & . & . & 40 & . \\
\hline \multicolumn{8}{|l|}{ Quercetalia pubescenti-petraeae } \\
\hline Convallaria majalis & E1 & 80 & . & 63 & 20 & 100 & . \\
\hline Lathyrus venetus & E1 & 70 & 15 & 75 & 100 & 40 & . \\
\hline Ostrya carpinifolia & $\mathrm{E} 3 \mathrm{~b}$ & 60 & 23 & 38 & 100 & 60 & 77 \\
\hline Ostrya carpinifolia & $\mathrm{E} 3 \mathrm{a}$ & 30 & . & 25 & 20 & 80 & 23 \\
\hline Ostrya carpinifolia & $\mathrm{E} 2 \mathrm{~b}$ & . & . & . & . & 40 & . \\
\hline Ostrya carpinifolia & $\mathrm{E} 2 \mathrm{a}$ & . & . & . & . & 20 & . \\
\hline Helleborus multifidus subsp. istriacus* & E1 & 50 & 8 & 88 & . & 80 & . \\
\hline Fraxinus ornus & $\mathrm{E} 3 \mathrm{~b}$ & . & 31 & 50 & 60 & 40 & 15 \\
\hline Fraxinus ornus & E3a & 60 & 8 & 75 & 40 & 80 & 85 \\
\hline Fraxinus ornus & $\mathrm{E} 2 \mathrm{~b}$ & 30 & 15 & 25 & . & 80 & 8 \\
\hline Fraxinus ornus & $\mathrm{E} 2 \mathrm{a}$ & 20 & 54 & 25 & 20 & 40 & 15 \\
\hline Fraxinus ornus & E1 & 40 & . & 25 & 20 & . & . \\
\hline Mercurialis ovata & E1 & 60 & . & 63 & . & 100 & . \\
\hline Melittis melissophyllum & E1 & 20 & . & 75 & . & 60 & . \\
\hline Cornus mas & $\mathrm{E} 2 \mathrm{~b}$ & 20 & 15 & 13 & 100 & 60 & 15 \\
\hline Tanacetum corymbosum & E1 & 20 & . & 88 & 20 & 40 & 8 \\
\hline Arabis turrita & E1 & 20 & 15 & . & 40 & 40 & . \\
\hline Asparagus tenuifolius & E1 & 20 & . & 50 & . & 20 & . \\
\hline Mercurialis x paxii & E1 & 20 & . & 25 & . & 20 & . \\
\hline Quercus cerris & $\mathrm{E} 3 \mathrm{~b}$ & 10 & . & 38 & . & . & . \\
\hline Sorbus aria (Aria edulis) & $\mathrm{E} 3 \mathrm{~b}$ & . & . & 50 & . & 40 & . \\
\hline Sorbus aria (Aria edulis) & E3a & 10 & . & 38 & 40 & 40 & 8 \\
\hline Sorbus aria (Aria edulis) & $\mathrm{E} 2 \mathrm{~b}$ & . & . & 13 & 20 & 40 & . \\
\hline Sorbus aria (Aria edulis) & $\mathrm{E} 2 \mathrm{a}$ & . & . & 13 & . & 20 & . \\
\hline Sorbus aria (Aria edulis) & E1 & . & . & 13 & . & . & . \\
\hline Euonymus verrucosa & $\mathrm{E} 2 \mathrm{~b}$ & . & . & 25 & . & . & . \\
\hline Euonymus verrucosa & $\mathrm{E} 2 \mathrm{a}$ & 10 & . & 50 & . & 60 & . \\
\hline Quercus pubescens & $\mathrm{E} 3 \mathrm{~b}$ & . & . & . & 20 & 40 & . \\
\hline Quercus pubescens & E3a & 10 & . & . & . & . & . \\
\hline Quercus pubescens & $\mathrm{E} 2 \mathrm{~b}$ & . & . & . & . & 20 & . \\
\hline Lathyrus niger & E1 & 10 & . & 13 & . & . & . \\
\hline Orchis pallens & E1 & 10 & . & . & 20 & . & . \\
\hline Hierochloë australis & E1 & 10 & . & . & . & 80 & . \\
\hline Aristolochia lutea & E1 & 10 & . & . & . & . & . \\
\hline Prunus mahaleb & $\mathrm{E} 2 \mathrm{~b}$ & $\cdot$ & . & . & . & 20 & . \\
\hline Prunus mahaleb & $\mathrm{E} 2 \mathrm{a}$ & 10 & . & . & . & . & . \\
\hline Viola sepincola & E1 & 10 & . & . & . & . & . \\
\hline Tamus communis & E1 & . & 8 & . & 20 & . & . \\
\hline Cnidium silaifolium & E1 & . & . & 100 & 60 & 20 & . \\
\hline Campanula persicifolia & E1 & . & . & 50 & . & 60 & . \\
\hline Hypericum montanum & E1 & . & . & 13 & . & 20 & . \\
\hline Orchis mascula subsp. speciosa & E1 & . & . & . & . & . & 8 \\
\hline
\end{tabular}




\begin{tabular}{|c|c|c|c|c|c|c|c|}
\hline \multicolumn{2}{|l|}{ Successive number (Zaporedna številka) } & 1 & 2 & 3 & 4 & 5 & 6 \\
\hline \multicolumn{8}{|l|}{ Quercetalia roboris } \\
\hline Castanea sativa & E3b & . & 8 & . & 100 & . & . \\
\hline Castanea sativa & E1 & . & 8 & . & . & . & . \\
\hline Rubus hirtus & E2 & . & 8 & . & . & . & 8 \\
\hline Serratula tinctoria & E1 & . & . & . & 20 & 20 & . \\
\hline \multicolumn{8}{|l|}{ Querco-Fagetea } \\
\hline Veratrum nigrum & E1 & 90 & 8 & 38 & 60 & 100 & 15 \\
\hline Hepatica nobilis & E1 & 80 & 46 & 88 & 80 & 100 & 15 \\
\hline Hedera helix & E3a & 70 & 92 & 13 & 80 & 40 & 15 \\
\hline Hedera helix & $\mathrm{E} 2 \mathrm{a}$ & . & . & . & . & 40 & . \\
\hline Hedera helix & E1 & 80 & 69 & 13 & 100 & 60 & 31 \\
\hline Lonicera xylosteum & E2 & 90 & . & 88 & . & 60 & 85 \\
\hline Carex digitata & E1 & 70 & 31 & 50 & . & 100 & 62 \\
\hline Anemone ranunculoides & E1 & 60 & . & . & . & . & 46 \\
\hline Corylus avellana & E3a & 10 & 15 & . & . & . & 62 \\
\hline Corylus avellana & $\mathrm{E} 2 \mathrm{~b}$ & 50 & 69 & 88 & 100 & 40 & 100 \\
\hline Corylus avellana & $\mathrm{E} 2 \mathrm{a}$ & 10 & . & 13 & 40 & . & . \\
\hline Corylus avellana & E1 & . & 15 & . & . & . & . \\
\hline Acer campestre & $\mathrm{E} 3 \mathrm{~b}$ & 40 & 46 & . & 40 & . & 38 \\
\hline Acer campestre & E3a & 60 & 38 & 75 & . & . & 23 \\
\hline Acer campestre & $\mathrm{E} 2 \mathrm{~b}$ & 20 & 15 & 25 & . & . & . \\
\hline Acer campestre & $\mathrm{E} 2 \mathrm{a}$ & 10 & . & 50 & . & . & . \\
\hline Acer campestre & E1 & 20 & 23 & 63 & 40 & . & 8 \\
\hline Viola mirabilis & E1 & 60 & . & . & . & . & . \\
\hline Melica uniflora & E1 & 50 & . & 50 & . & . & . \\
\hline Lathraea squamaria & E1 & 30 & 23 & . & 20 & . & 15 \\
\hline Quercus petraea & $\mathrm{E} 3 \mathrm{~b}$ & 40 & 15 & . & 40 & 20 & . \\
\hline Quercus petraea & E1 & . & 8 & . & 20 & . & . \\
\hline Gagea lutea & E1 & 40 & 8 & . & . & . & 38 \\
\hline Stellaria holostea & E1 & 30 & . & . & . & . & . \\
\hline Ranunculus ficaria & E1 & 20 & 46 & 13 & . & . & . \\
\hline Anemone nemorosa & E1 & 20 & 77 & . & 40 & . & 85 \\
\hline Viola riviniana & E1 & 20 & . & . & . & 20 & 15 \\
\hline Moehringia trinervia & E1 & 10 & 8 & 25 & . & . & 15 \\
\hline Dactylis glomerata subsp. lobata (D. polygama) & E1 & 10 & . & . & . & . & 38 \\
\hline Ulmus minor & $\mathrm{E} 2 \mathrm{a}$ & 10 & . & . & . & 20 & . \\
\hline Aegopodium podagraria & E1 & . & 92 & 38 & . & . & 54 \\
\hline Crataegus laevigata & $\mathrm{E} 2 \mathrm{~b}$ & . & 38 & . & . & . & . \\
\hline Crataegus laevigata & $\mathrm{E} 2 \mathrm{a}$ & . & . & . & 40 & . & . \\
\hline Clematis vitalba & E3a & . & 8 & . & 60 & . & . \\
\hline Clematis vitalba & $\mathrm{E} 2 \mathrm{a}$ & . & 46 & 63 & . & . & . \\
\hline Clematis vitalba & E1 & . & . & 13 & 40 & . & 15 \\
\hline Cerastium sylvaticum & E1 & . & 23 & . & . & . & . \\
\hline Scilla bifolia & E1 & . & 15 & 75 & . & . & . \\
\hline Malus sylvestris & E3a & . & . & 13 & . & . & . \\
\hline Malus sylvestris & $\mathrm{E} 2 \mathrm{~b}$ & . & 8 & . & . & . & 8 \\
\hline Pyrus pyraster & $\mathrm{E} 2 \mathrm{a}$ & . & . & 13 & . & . & 8 \\
\hline Rosa arvensis & $\mathrm{E} 2 \mathrm{a}$ & . & . & 13 & . & . & . \\
\hline Spiraea chamaedryfolia & $\mathrm{E} 2 \mathrm{a}$ & . & . & . & 20 & . & . \\
\hline
\end{tabular}




\begin{tabular}{|c|c|c|c|c|c|c|c|}
\hline Successive number (Zaporedna številka) & & 1 & 2 & 3 & 4 & 5 & 6 \\
\hline Festuca heterophylla & E1 & . & . & . & . & . & 38 \\
\hline Listera ovata & E1 & . & . & . & . & . & 31 \\
\hline Dactylorhiza fuchsii & E1 & . & . & . & . & . & 23 \\
\hline Vinca minor & E1 & . & . & . & . & . & 15 \\
\hline Cruciata glabra & E1 & . & . & . & . & . & 15 \\
\hline \multicolumn{8}{|l|}{ Vaccinio-Piceetea } \\
\hline Solidago virgaurea & E1 & 20 & . & 63 & . & 40 & . \\
\hline Maianthemum bifolium & E1 & 20 & . & 13 & . & . & . \\
\hline Hieracium murorum & E1 & 10 & . & . & . & . & . \\
\hline Aposeris foetida & E1 & . & 15 & 25 & 60 & . & . \\
\hline Oxalis acetosella & E1 & . & 54 & . & . & . & . \\
\hline Picea abies & $\mathrm{E} 3 \mathrm{~b}$ & . & . & . & . & . & . \\
\hline Picea abies & $\mathrm{E} 2 \mathrm{~b}$ & . & . & 25 & . & . & . \\
\hline Rosa pendulina & $\mathrm{E} 2 \mathrm{a}$ & . & . & 13 & . & . & . \\
\hline \multicolumn{8}{|l|}{ Erico-Pinetea } \\
\hline Calamagrostis varia & E1 & . & . & 13 & . & . & 31 \\
\hline Amelanchier ovalis & $\mathrm{E} 2 \mathrm{a}$ & . & . & . & . & 40 & . \\
\hline Chamaecytisus hirsutus & E1 & . & . & . & . & 40 & . \\
\hline Pinus nigra & $\mathrm{E} 3 \mathrm{~b}$ & . & . & . & . & 20 & 8 \\
\hline Peucedanum austriacum & E1 & . & . & . & . & . & 23 \\
\hline Rubus saxatilis & E1 & . & . & . & . & . & 8 \\
\hline Carex alba & E1 & . & . & . & . & . & 8 \\
\hline \multicolumn{8}{|l|}{ Sambuco-Salicion capreae } \\
\hline Salix caprea & E3b & . & . & . & . & . & 8 \\
\hline Salix caprea & E3a & . & . & . & . & . & 15 \\
\hline Betula pendula & $\mathrm{E} 3 \mathrm{~b}$ & . & . & . & . & . & 8 \\
\hline Sorbus aucuparia & $\mathrm{E} 3 \mathrm{~b}$ & . & . & . & . & . & 8 \\
\hline \multicolumn{8}{|l|}{ Rhmano-Prunetea } \\
\hline Euonymus europaed & E2 & 20 & 38 & 50 & . & . & 8 \\
\hline Berberis vulgaris & E2a & 10 & . & . & 20 & . & 8 \\
\hline Robinia pseudoacacia & E3b & . & 46 & . & 40 & . & . \\
\hline Robinia pseudoacacia & E3a & . & 8 & . & 40 & . & . \\
\hline Robinia pseudoacacia & E2a & . & 8 & . & . & . & . \\
\hline Crataegus monogyna & E3a & . & 8 & . & 20 & . & 15 \\
\hline Crataegus monogyna & $\mathrm{E} 2 \mathrm{~b}$ & . & . & . & 60 & 20 & 15 \\
\hline Cornus sanguinea & E2b & . & . & 13 & . & . & . \\
\hline Cornus sanguinea & E2a & . & . & 13 & . & . & . \\
\hline Rubus fruticosus agg. & $\mathrm{E} 2 \mathrm{a}$ & . & . & . & 40 & . & . \\
\hline Rosa canina & E2a & . & . & . & . & 20 & . \\
\hline \multicolumn{8}{|l|}{ Mulgedio-Aconitetea, Betulo-Alnetea } \\
\hline Aconitum lycoctonum & E1 & 30 & 38 & 75 & . & . & 15 \\
\hline Senecio nemorensis & E1 & 20 & 15 & . & 40 & . & . \\
\hline Senecio ovatus & E1 & 10 & . & 38 & 20 & . & 54 \\
\hline Ribes alpinum & $\mathrm{E} 2 \mathrm{a}$ & 10 & . & . & . & . & . \\
\hline Athyrium filix-femina & E1 & . & 31 & . & . & . & 15 \\
\hline Aconitum variegatum & E1 & . & . & 25 & . & . & . \\
\hline Saxifraga rotundifolia & E1 & . & . & 75 & . & . & . \\
\hline Silene dioica & E1 & . & . & 25 & . & . & . \\
\hline Milium effusum & E1 & . & . & . & . & . & 8 \\
\hline
\end{tabular}




\begin{tabular}{|c|c|c|c|c|c|c|c|}
\hline Successive number (Zaporedna številka) & & 1 & 2 & 3 & 4 & 5 & 6 \\
\hline Polygonatum verticillatum & E1 & . & . & . & . & . & 8 \\
\hline \multicolumn{8}{|l|}{ Epilobietea angustifolii } \\
\hline Solanum dulcamara & E1 & 10 & . & . & . & . & . \\
\hline Carpesium cernuum & E1 & . & 23 & . & . & . & . \\
\hline Galeopsis pubescens & E1 & . & 8 & . & . & . & . \\
\hline Physalis alkekengi & E1 & . & 8 & . & . & . & . \\
\hline Arctium nemorosum & E1 & . & 8 & 25 & . & . & . \\
\hline Stachys sylvatica & E1 & . & 8 & . & . & . & . \\
\hline Fragaria vesca & E1 & . & . & 50 & . & . & 46 \\
\hline Hypericum hirsutum & E1 & . & . & 50 & . & . & . \\
\hline Lapsana communis & E1 & . & . & 25 & . & . & . \\
\hline \multicolumn{8}{|l|}{ Galio-Urticetea } \\
\hline Urtica dioica & E1 & 20 & 8 & 25 & . & . & 31 \\
\hline Geum urbanum & E1 & . & 15 & 63 & . & . & 62 \\
\hline Parietaria officinalis & E1 & . & 8 & . & . & . & . \\
\hline Lamium maculatum & $\mathrm{E} 1$ & . & . & 38 & . & . & . \\
\hline Alliaria petiolata & E1 & . & . & 13 & . & . & 8 \\
\hline Torilis japonica & E1 & . & . & 13 & . & . & . \\
\hline Allium vineale & E1 & . & . & . & . & . & 8 \\
\hline \multicolumn{8}{|l|}{ Stellarietea mediae } \\
\hline Stellaria neglecta & E1 & 20 & . & . & . & . & . \\
\hline Galium aparine & E1 & . & 8 & 13 & . & . & . \\
\hline Erigeron annuus & E1 & . & 8 & . & . & . & . \\
\hline Myosotis arvensis & E1 & . & . & 13 & . & . & . \\
\hline Myosotis sp. & E1 & . & . & 13 & . & . & . \\
\hline \multicolumn{8}{|l|}{ Trifolio-Geranietea } \\
\hline Campanula rapunculoides & E1 & 60 & . & 75 & 60 & 60 & 23 \\
\hline Lilium carniolicum & E1 & 20 & . & 38 & . & 20 & . \\
\hline Viola odorata & E1 & 10 & . & . & . & . & . \\
\hline Melampyrum velebiticum & E1 & 10 & . & . & . & 40 & . \\
\hline Verbascum lanatum & E1 & . & . & 25 & . & . & . \\
\hline Verbascum chaixii & E1 & . & . & 25 & . & . & . \\
\hline Polygonatum odoratum & E1 & . & . & 13 & . & 40 & . \\
\hline Vincetoxicum hirundinaria & E1 & . & . & 13 & 20 & 20 & . \\
\hline Paeonia officinalis & E1 & . & . & 13 & . & 40 & . \\
\hline Silene nutans & E1 & . & . & 13 & . & 20 & . \\
\hline Valeriana nemorensis & E1 & . & . & 13 & . & 40 & . \\
\hline Thalictrum minus & E1 & . & . & 13 & $\cdot$ & . & . \\
\hline Vicia sp. & E1 & . & . & 13 & . & . & . \\
\hline Vicia sylvatica & E1 & . & . & 13 & . & . & . \\
\hline Calamintha einseleana & E1 & . & . & 13 & . & . & . \\
\hline Anthericum ramosum & E1 & . & . & . & . & 40 & . \\
\hline Digitalis grandiflora & E1 & . & . & . & . & 40 & . \\
\hline Viola hirta & E1 & . & . & . & . & 20 & . \\
\hline Peucedanum cervaria & E1 & . & . & . & . & 20 & . \\
\hline Valeriana wallrothii (V. collina) & E1 & . & . & . & . & 20 & 8 \\
\hline Lilium bulbiferum & E1 & & . & . & . & . & 8 \\
\hline \multicolumn{8}{|l|}{ Festuco-Brometea } \\
\hline Allium carinatum subsp. carinatum & E1 & 10 & . & . & . & . & 23 \\
\hline
\end{tabular}




\begin{tabular}{|c|c|c|c|c|c|c|c|}
\hline Successive number (Zaporedna številka) & & 1 & 2 & 3 & 4 & 5 & 6 \\
\hline Taraxacum laevigatum & E1 & . & . & 25 & . & . & . \\
\hline Brachypodium rupestre & E1 & . & . & 13 & . & . & . \\
\hline Arabis hirsuta & E1 & . & . & 13 & . & . & . \\
\hline Sesleria tenuifolia & E1 & . & . & . & . & 40 & . \\
\hline Carex humilis & E1 & . & . & . & . & 20 & . \\
\hline Iberis linifolia & E1 & . & . & . & . & 20 & . \\
\hline Cirsium erisithales & E1 & . & . & . & . & . & 23 \\
\hline Euphorbia cyparissias & E1 & . & . & . & . & . & 8 \\
\hline \multicolumn{8}{|l|}{ Koelerio-Corynephoretea } \\
\hline Cardaminopsis arenosa & E1 & 10 & . & 25 & 20 & 60 & 8 \\
\hline Saxifraga tridactylites & E1 & . & . & . & . & 20 & . \\
\hline \multicolumn{8}{|l|}{ Molinio-Arrbenatheretea } \\
\hline Veronica chamaedrys & E1 & 10 & . & 88 & . & . & 23 \\
\hline Muscari botryoides & E1 & 10 & . & . & . & . & . \\
\hline Anthriscus sylvestris & E1 & 10 & . & . & . & . & 8 \\
\hline Taraxacum sect. Ruderalia & E1 & . & . & 38 & 20 & . & . \\
\hline Angelica sylvestris & E1 & . & . & . & . & . & 46 \\
\hline Colchicum autumnale & E1 & . & . & . & . & . & 8 \\
\hline Crocus albiflorus & E1 & . & . & . & . & . & 8 \\
\hline Poa trivialis & E1 & . & . & . & . & . & 8 \\
\hline Galium mollugo & E1 & . & . & . & . & . & 8 \\
\hline \multicolumn{8}{|l|}{ Cystopteridion fragilis } \\
\hline Moehringia muscosa & E1 & 30 & 8 & 63 & 60 & 100 & 23 \\
\hline Pseudofumaria alba & E1 & 30 & . & 75 & . & . & . \\
\hline Cystopteris fragilis & E1 & 10 & . & 25 & . & . & . \\
\hline \multicolumn{8}{|c|}{ Physoplexido-Saxifragion, Potentilletalia caulescentis } \\
\hline Campanula pyramidalis & E1 & 10 & 15 & 63 & . & 60 & . \\
\hline Micromeria thymifolia & E1 & . & . & . & . & 40 & . \\
\hline Primula auricula & E1 & . & . & . & . & 40 & . \\
\hline Hieracium lasiophyllum & E1 & . & . & . & . & 20 & . \\
\hline \multicolumn{8}{|l|}{ Asplenietea trichomanis } \\
\hline Asplenium trichomanes & E1 & 30 & 46 & 50 & 80 & 80 & 77 \\
\hline Polypodium vulgare & E1 & 30 & 23 & 13 & 20 & 20 & 38 \\
\hline Polypodium interjectum & E1 & 10 & 38 & . & . & 40 & . \\
\hline Asplenium ruta-muraria & E1 & 10 & . & 25 & . & 40 & 8 \\
\hline Asplenium adiantum-nigrum & E1 & 10 & . & . & . & . & . \\
\hline Sedum maximum & E1 & . & . & 50 & . & 60 & . \\
\hline Ceterach officinarum & E1 & . & . & 25 & . & . & . \\
\hline Polypodium australe & E1 & . & . & . & . & 20 & . \\
\hline Parietaria judaica & E1 & . & . & . & . & 20 & . \\
\hline Cymbalaria muralis & E1 & . & . & . & . & . & 15 \\
\hline Phyteuma scheuchzeri subsp. columnae & E1 & . & . & . & . & . & 8 \\
\hline \multicolumn{8}{|l|}{ Mosses and lichens (Mahovi in lišaji) } \\
\hline Isothecium alopecuroides & E0 & 80 & 69 & 25 & 80 & . & 85 \\
\hline Brachythecium rutabulum & E0 & 80 & 23 & . & . & 20 & 38 \\
\hline Ctenidium molluscum & E0 & 70 & 38 & 63 & 60 & 100 & 85 \\
\hline Neckera crispa & E0 & 70 & 15 & 50 & 100 & 80 & 46 \\
\hline Thamnobryum alopecurum & E0 & 70 & 85 & . & . & . & 31 \\
\hline Plagiomnium undulatum & E0 & 70 & 8 & . & . & 100 & 31 \\
\hline
\end{tabular}




\begin{tabular}{|c|c|c|c|c|c|c|c|}
\hline Successive number (Zaporedna številka) & & 1 & 2 & 3 & 4 & 5 & 6 \\
\hline Homalothecium lutescens & E0 & 60 & 15 & 88 & 20 & 40 & 46 \\
\hline Peltigera canina & E0 & 50 & . & 25 & . & 40 & 8 \\
\hline Anomodon viticulosus & E0 & 30 & 38 & 63 & 40 & 40 & 38 \\
\hline Neckera complanata & E0 & 30 & 23 & 33 & 20 & 20 & 23 \\
\hline Anomodon attenuatus & E0 & 30 & 15 & . & . & 20 & 8 \\
\hline Polytrichum formosum & E0 & 10 & . & 13 & . & . & 46 \\
\hline Eurhynchium striatum & E0 & 10 & . & . & . & . & . \\
\hline Thuidium delicatulum & E0 & . & 8 & . & . & 20 & . \\
\hline Conocephalum conicum & E0 & . & 8 & . & . & . & . \\
\hline Fissidens taxifolius & E0 & . & 8 & . & . & . & . \\
\hline Schistidium apocarpum & E0 & . & . & 63 & 60 & 20 & 38 \\
\hline Plagiochila porelloides & E0 & . & . & 25 & . & . & . \\
\hline Porella platyphylla & E0 & . & . & 25 & . & . & 31 \\
\hline Homalothecium sericeum & E0 & . & . & 13 & . & . & 8 \\
\hline Mnium sp. & E0 & . & . & 13 & . & . & 8 \\
\hline Dicranum scoparium & E0 & . & . & . & . & 60 & . \\
\hline Thuidium tamariscinum & E0 & . & . & . & . & 60 & 8 \\
\hline Hylocomium splendens & E0 & . & . & & . & 40 & . \\
\hline Rhytidiadelphus triquetrus & E0 & . & . & . & . & 40 & . \\
\hline Tortella tortuosa & E0 & . & . & . & . & 20 & . \\
\hline Plagiomnium cuspidatum & E0 & . & . & . & . & . & 46 \\
\hline Homalothecium philippeanum & E0 & . & . & . & . & . & 31 \\
\hline Brachythecium salebrosum & E0 & . & . & . & . & . & 15 \\
\hline Atrichum undulatum & E0 & . & - & . & - & . & 15 \\
\hline Porella arboris-vitae & E0 & $\cdot$ & . & & . & . & 8 \\
\hline Fissidens dubius & E0 & . & . & . & . & . & 8 \\
\hline
\end{tabular}

\section{Legend / Legenda}

1 Corydalido ochroleucae-Aceretum pseudoplatani veratretosum nigri

2 Fraxino orni-Aceretum pseudoplatani

3 Paeonio officinalis-Tilietum platyphylli saxifragetosum rotundifoliae

4 Paeonio officinalis-Tilietum platyphylli castaneetosum sativae

5 Paeonio officinalis-Tilietum platyphylli hierochloetosum australis

6 Lamio orvalae-Tilietum platyphylli

Crocus vernus subsp. vernus* is according to new findings Crocus heuffellianus Herb. - Peruzzi (2016)

Helleborus multifidus subsp. istriacus* (incl. Helleborus dumetorum subsp. istriacus) - Rottensteiner (2016) 